\title{
Simulation of Gas Detectors Using Ramo's Theorem
}

by by

\author{
Dylan Pizzi
}

\author{
A thesis submitted to the \\ Faculty of Graduate and Postdoctoral Affairs \\ in partial fulfilment of the requirements \\ for the degree of
}

\author{
Master of Science \\ in
}

Particle Physics

\author{
Ottawa-Carleton Institute for Physics \\ Department of Physics \\ Carleton University \\ Ottawa, Ontario \\ (C) 2019 \\ Dylan Pizzi
}




\section{Abstract}

Between 2019 and 2021, major upgrades are required to the ATLAS detector and its muon system to prepare for the high-luminosity phase of operation of the Large Hadron Collider (LHC). One upgrade will be the replacement of the present Small Wheel with the New Small Wheel (NSW) that will allow improved tracking and triggering on muons originating from the interaction point. One half of the NSW upgrade is based on the gaseous small-strip Thin Gap Chamber (sTGC) multiwire proportional chamber (MWPC) technology. As particles traverse the gaseous region they produce ionization clusters which drift towards the anodes and cathodes. Ultimately, the avalanche on the wires induces charge on the detector elements of the MWPC. A method to determine this induced charge signal using Ramo's theorem will be described. An explanation of the physics inherent to a MWPC will also be summarized. Subsequently, an overview of the sTGC will be introduced, leading into a description of its simulation. The effects of detector components on signal formation will be presented, followed by how they are taken into account in the simulations. As a proof of concept, a comparison of the simulation results to test beam data will be shown. 


\section{Acknowledgements}

Throughout the course of this work many people played a role in its completion, but I would like to thank a few. First and foremost, I would like to express my deepest gratitude to my supervisor Alain Bellerive for teaching me all about the intricacies of the sTGC and for the support and guidance over the course of this work, especially the writing of this thesis. I would like to thank our collaborators in the ATLAS Muon Group for providing the test beam data so that we could effectively test the validity

of our simulation. Furthermore, I would like to thank Alexandre Laurier, your help in explaining the code to analyze the test beam data proved to be invaluable. Also, thank you to my friends here at Carleton, you guys definitely made getting through this Master's a little easier, cheers! To my parents, John and Charmyn, I have the most heartfelt thanks for always pushing me to pursue my goals, without you I would not be where I am today. To my dog, Simon, I would like to thank you for showing me that sometimes in order to solve the problem its OK to step away from it and take a break. Last, but most important, I would like to thank my amazing partner, Whitney, who is always there to support me when I need it, thank you for everything you do. 


\section{Table of Contents}

Abstract $\quad$ i

Acknowledgements $\quad$ ii

Table of Contents iii

List of Tables $\quad$ vi

List of Figures $\quad$ vii

List of Abbreviations $\quad$ x

1 Introduction 1

1.1 The Large Hadron Collider $(\mathrm{LHC}) \ldots \ldots$. . . . . . . . . . 1

1.2 The ATLAS Detector . . . . . . . . . . . . . . . . . 3

1.3 New Small Wheel $(\mathrm{NSW}) \ldots \ldots . \ldots$. . . . . . . . . . 6

1.4 Motivation . . . . . . . . . . . . . . . . 10

1.5 Thesis Layout . . . . . . . . . . . . . . . . . . 11

2 Charge Induction and Ramo's Theorem 13

2.1 Reciprocity Theorem ................... . . 13

2.2 Stationary Charge . . . . . . . . . . . . . . . 18 
2.3 Moving Charge . . . . . . . . . . . . . . . . . . . . . . . . . . 23

3 Physics of a MWPC 28

3.1 Fundamentals of a MWPC . . . . . . . . . . . . . . . 28

3.2 Ionization and Transport Properties . . . . . . . . . . . 30

3.2 .1 Ionization . . . . . . . . . . . . . . . . . 30

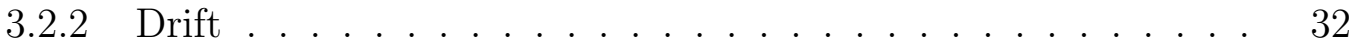

3.2 .3 Wire Amplification . . . . . . . . . . . . . . 36

3.3 Charge Dispersion on Resistive Layer . . . . . . . . . . . . . 38

3.3 .1 Symmetric Ground . . . . . . . . . . . . . . . . . . . 38

3.3 .2 Ground on One Side . . . . . . . . . . . . . . . . . . 42

4 Small-Strip Thin Gap Chamber 46

4.1 Overview of the sTGC $\ldots \ldots \ldots \ldots \ldots$

4.2 Simulation . . . . . . . . . . . . . . . . . . . 51

4.3 Anode Signal . . . . . . . . . . . . . . . . . . . 54

4.3.1 Time of Arrival of the Electron Clusters . . . . . . . . . 54

4.3 .2 Anode Electronics Response . . . . . . . . . . . . . . . 57

4.4 Cathode Signal . . . . . . . . . . . . . . . . . . . . . . . . . . 59

4.4 .1 Signal Production on Strips _. . . . . . . . . . . . . 59

$4.4 .2 \quad$ Signal Processing . . . . . . . . . . . . . . . . . 59

5 Track Reconstruction $\quad 66$

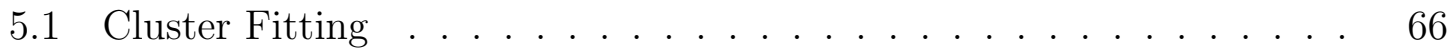

5.2 Y-Coordinate Measurement . . . . . . . . . . . . . 71

6 RC Time Constant $\quad 75$

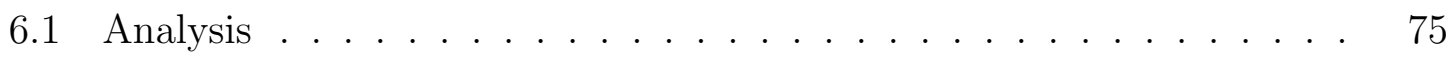

6.2 Determination of $\mathrm{RC} \ldots \ldots \ldots \ldots \ldots \ldots$ 
7 Conclusion \& Outlook $\quad 85$

$\begin{array}{lr}\text { References } & 87\end{array}$

$\begin{array}{ll}\text { A Resistive Layer Response Functions } & 90\end{array}$ 


\section{List of Tables}

4.1 sTGC design parameters . . . . . . . . . . . . . . 48

5.1 Results from shape test . . . . . . . . . . . . . . . . 69

6.1 Best fit time constant estimate . . . . . . . . . . . . 82

6.2 Ratio of charge on neighbouring strips relative to central strip . . . . 84 


\section{List of Figures}

1.1 Diagram of LHC and its four major experiments . . . . . . . . . . 2

1.2 Diagram of the ATLAS experiment . . . . . . . . . . . 3

1.3 Diagram of the ATLAS Muon Spectrometer . . . . . . . . . . 5

1.4 Higgs boson to four muon event candidate . . . . . . . . . . . . 7

1.5 NSW location within ATLAS . . . . . . . . . . . . . 8

1.6 Highlight of false muon triggers . . . . . . . . . . . . . . . . . . . . 8

1.7 NSW sector breakdown . . . . . . . . . . . . . . . . . . . . 9

1.8 NSW sectors and components . . . . . . . . . . . . . . . . 10

2.1 Two conducting surfaces producing an electric field . . . . . . . . 13

2.2 System of grounded conductors with stationary point charge . . . . . 19

2.3 Alternate systems used to determine charge induced on electrodes . . 21

2.4 System of electrodes with moving charged particle . . . . . . . . . . . 24

2.5 Equipotential lines of the weighted field in a two-electrode plane configuration ............................. 25

3.1 Traditional MWPC design . . . . . . . . . . . . . . . . 28

3.2 Electric field and equipotential lines in a MWPC . . . . . . . . . . 29

3.3 Ion Pair Collection in Proportional Counters . . . . . . . . . . . . . 31 
3.4 Electron drift velocities and longitudinal diffusion coefficients in mixtures of argon and $\mathrm{CO}_{2} \ldots \ldots \ldots \ldots$. . . . . . . . . . . . 35

3.5 Electron avalanche illustration . . . . . . . . . . . . . . 36

3.6 Townsend coefficients for several gases as a function of electric field . 38

3.7 Illustration of isotropic charge dispersion . . . . . . . . . . . . . . . . 39

3.8 Comparison of simulated and observed signal in MPGD . . . . . . . . 42

3.9 Electric potential of system using image charges . . . . . . . . . . . 43

3.10 Electric field lines for charge near an infinite grounded plane . . . . . 44

3.11 Charge dispersion with ground on one side . . . . . . . . . . 45

4.1 Illustration of muon passing through an sTGC quadruplet . . . . . . 46

4.2 sTGC cross-section . . . . . . . . . . . . . . . . . 47

4.3 Illustration of cathode strips . . . . . . . . . . . . . . . . 49

4.4 Drift velocity and electron cluster arrival time in sTGC . . . . . . . . 50

4.5 Cross-sectional view of the sTGC layout in Garfield . . . . . . . . . . 52

4.6 Steps to simulate signals on wires and strips . . . . . . . . . . . 53

4.7 Comparison of time of arrival of raw signals . . . . . . . . . . . 55

4.8 Zoom on a raw wire signal . . . . . . . . . . . . . . . 55

4.9 Comparison of simulated time of arrival with other results . . . . . 56

4.10 Convolution of raw wire signal with VMM shaping function . . . . . . 58

4.11 Process of charge induction in sTGC . . . . . . . . . . . . 60

4.12 Convolution of sTGC strip resulting in charge dispersion as a function of time . . . . . . . . . . . . . . . . . . 61

4.13 Convolution of sTGC strip resulting in charge dispersion to its second neighbour strip as a function of time . . . . . . . . . . . 63

4.14 Final simulated strip signal compared with test beam result . . . . . 64

5.1 Simulated cathode strip signals . . . . . . . . . . . . . 67 
5.2 Distribution of charge cluster over strips . . . . . . . . . . . . . 68

5.3 Charge cluster over strips for track reconstruction with Gaussian fits . 68

5.4 Reconstruction of $y$-coordinate using a weighted mean . . . . . . . . . 72

5.5 Reconstruction of $y$-coordinate using a Gaussian fit . . . . . . . . . . 73

6.1 Comparison of charge two charge clusters using $R C 21 \mathrm{~ns} / \mathrm{mm}^{2}$ and $120 \mathrm{~ns} / \mathrm{mm}^{2} \ldots \ldots \ldots \ldots \ldots \ldots$

6.2 Average charge distributions from sTGC test beam data . . . . . . 77

6.3 Average energy loss for particles in a 50:50 mixture of n-pentane: $\mathrm{CO}_{2} \quad 78$

6.4 Log-likelihood curves for the best fit estimator of $R C$. . . . . . . . . 81

6.5 Simulated distributions overlaid with test beam data . . . . . . . 83 


\title{
List of Abbreviations
}

\author{
ADC Analogue-to-Digital Converter \\ ASIC Application-Specific Integrated Circuit \\ ATLAS A Toroidal LHC Apparatus \\ BEM Boundary Element Method \\ BW Big Wheel \\ CSC Cathode Strip Chambers \\ FNAL Fermi National Laboratory \\ HL-LHC High-Luminosity LHC \\ ILC International Linear Collider \\ ILD International Linear Detector \\ IP Interaction Point \\ LAr Liquid Argon \\ LHC Large Hadron Collider \\ MDT Monitored Drift Tubes \\ MM Micromegas \\ MPGD Micro Pattern Gas Detector \\ MWPC Multiwire Proportional Chamber \\ NSW New Small Wheel \\ PDO Peak Detector Output \\ RPC Resistive Plate Chambers
}


SCT Semiconductor Tracker

SM Standard Model of particle physics

sTGC small-strip Thin Gap Chamber

SW Small Wheel

TGC Thin Gap Chambers

TPC Time Projection Chamber

TRT Transition Radiation Tracker 


\section{Chapter 1}

\section{Introduction}

\subsection{The Large Hadron Collider (LHC)}

The Large Hadron Collider (LHC) is a proton-proton circular accelerator located beneath the French-Swiss border near Geneva, Switzerland. The main goal of the LHC is to further our understanding of the Standard Model of particle physics (SM), as well as to probe for new physics not predicted by the SM. The SM predicts all known matter in the universe (quarks, leptons, and their respective anti-particles) and the mediators of three of the four fundamental forces of nature (gluon, W and $\mathrm{Z}$ bosons, and the photon). The final prediction of the SM was the Higgs boson which couples to all massive particles and was found at the LHC in 2012 [1]. Particle physicists want to study the Higgs boson in greater detail and search for signs of new physics, but this will require upgrades to the LHC and its detectors.

Shown in Figure 1.1, the LHC is a $27 \mathrm{~km}$ circumference ring and is at a depth ranging between $50 \mathrm{~m}$ to $175 \mathrm{~m}$ underground. Inside the ring are two separate beam lines that accelerate protons in opposite directions until they collide at the Interaction Points (IP's) within the detectors. By the end of 2018, proton beams were being accelerated to a centre-of-mass energy, $E_{\mathrm{CM}}$, of $13 \mathrm{TeV}$ with a luminosity of 


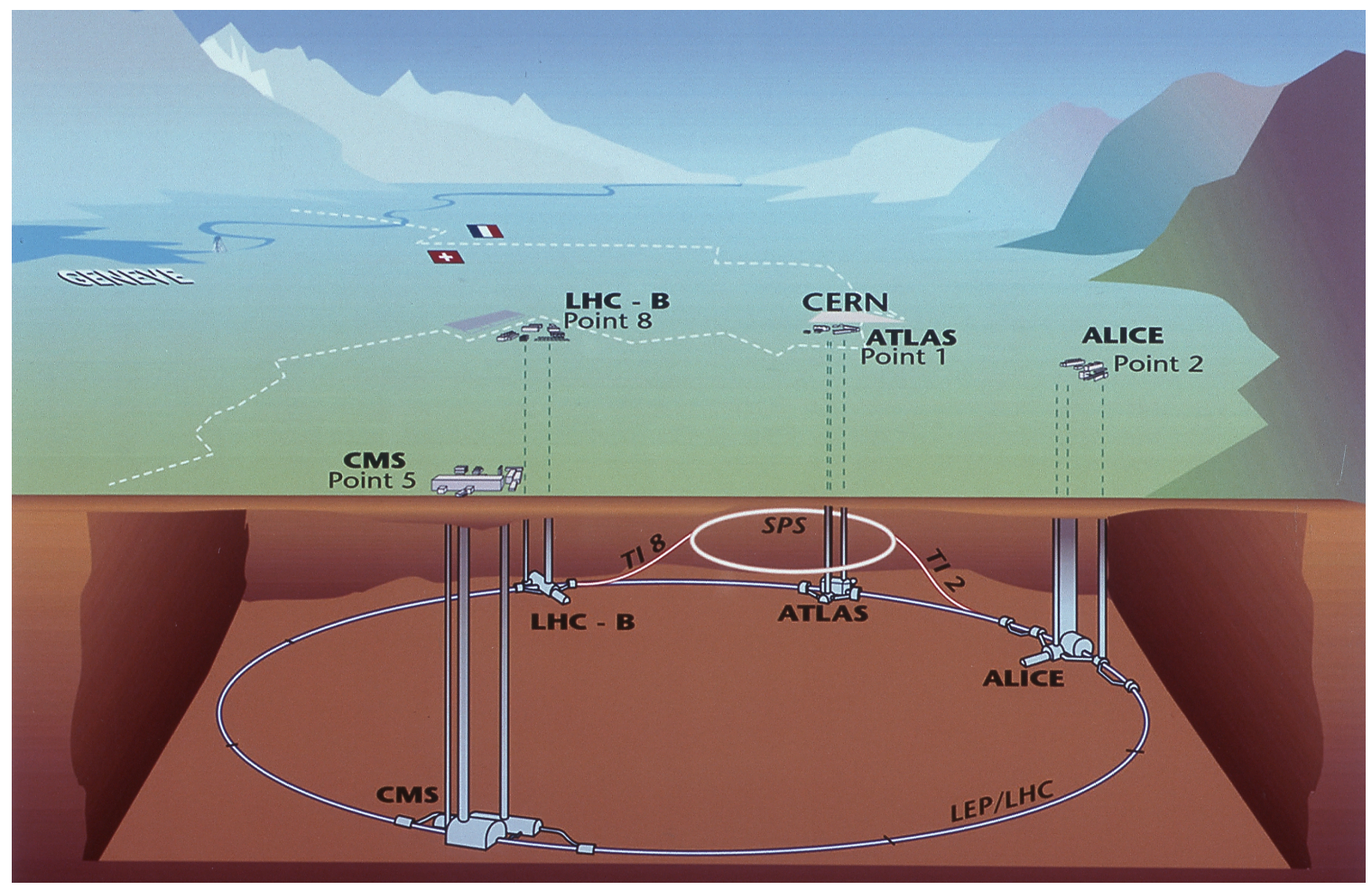

Figure 1.1: Illustration of the LHC ring beneath France-Switzerland border. Shown are the four main experiments along the ring as well as the LHC injector, the Super Proton Synchrotron (SPS) [3].

$2 \times 10^{34} \mathrm{~cm}^{-2} \mathrm{~s}^{-1}[2]$. The centre-of-mass energy refers to the total energy of a collision in the reference frame of the lab where each proton has an energy of $6.5 \mathrm{TeV}$. Furthermore, the beam is not a continuous stream of protons but is comprised of periodic bunches of protons moving around the ring. The luminosity refers to how many protons pass through a cross-sectional area every second. Therefore a greater luminosity means the denser the proton bunches and a higher probability of collisions.

To further study the Higgs boson and search for potential new physics, the LHC is undergoing scheduled upgrades to increase its design luminosity of $10^{34} \mathrm{~cm}^{-2} \mathrm{~s}^{-1}$ by a factor of about 10 to become the High-Luminosity LHC (HL-LHC). This will significantly increase the number of collisions within the detectors and hence the amount of radiation that passes through them. One of the main proton-proton experiments, 
A Toroidal LHC Apparatus (ATLAS) shown in Figure 1.1, will undergo several upgrades over the next seven years to prepare for the high luminosity environment.

\subsection{The ATLAS Detector}

The ATLAS detector is designed to study the physics resulting from collisions at the maximum LHC energy of $14 \mathrm{GeV}$. It is $45 \mathrm{~m}$ long, $25 \mathrm{~m}$ tall and made up of 4 main components: the inner detector, the calorimeter, the muon spectrometer, and the magnet system. Figure 1.2 shows the ATLAS detector and its main components broken down into their respective subsections.

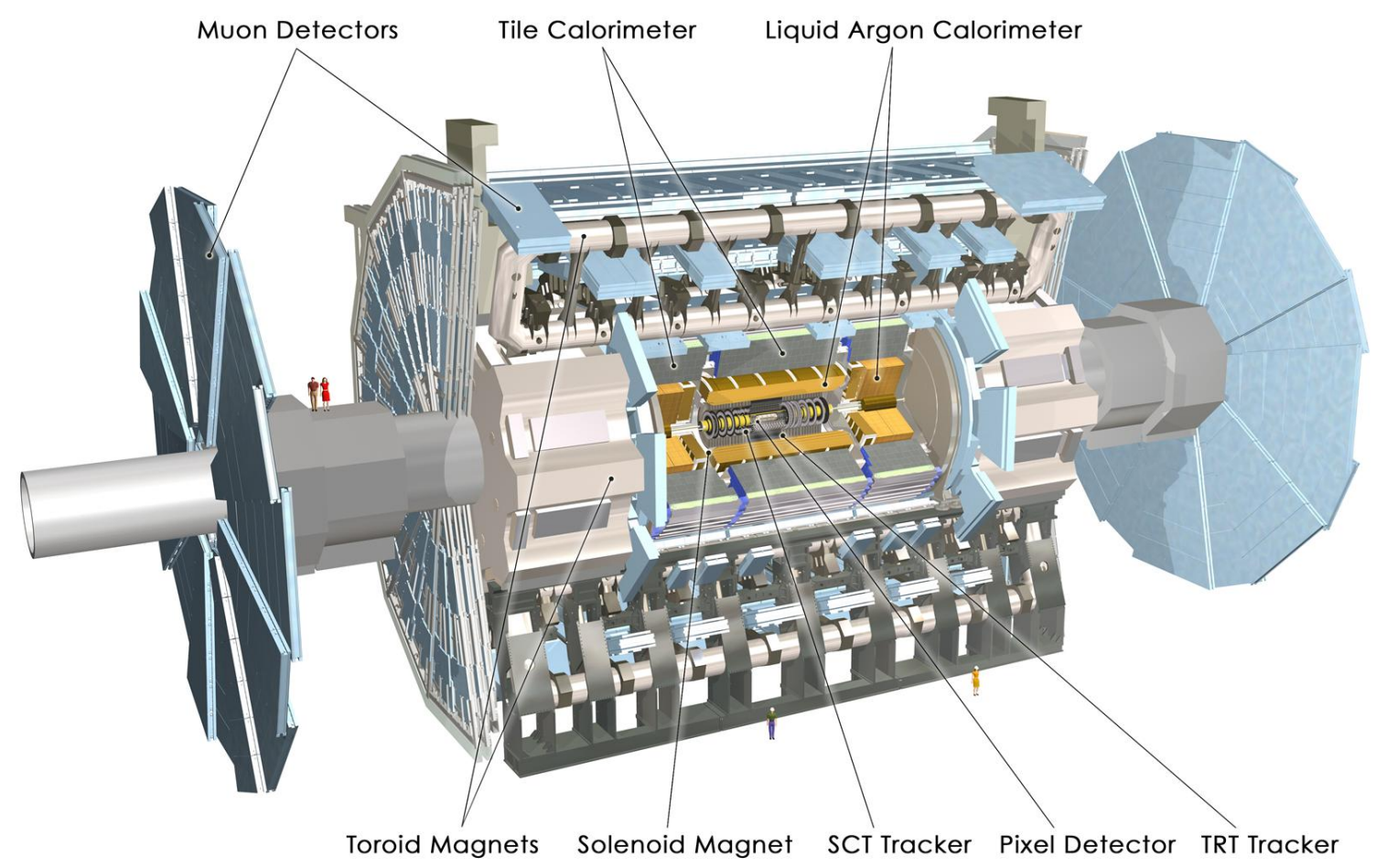

Figure 1.2: Illustration of ATLAS showing the major components of the detector [4].

The coordinate system in ATLAS defines the central beamline as the $z$-axis, the $x$-axis lies along the width of the detector, and the $y$-axis along its height. More commonly, the Lorentz invariant angle $\eta$, also known as the pseudorapidity, is used 
to describe a track's angle from the $z$-axis in the $y z$-plane with $|\eta|=\infty$ corresponding to the $z$-axis and $\eta=0$ being the $y$-axis. The pseudorapidity is related to the angle $\theta$ from the $z$-axis in Cartesian coordinates by

$$
\eta=-\ln \left[\tan \left(\frac{\theta}{2}\right)\right] .
$$

Furthermore, the angle $\phi$ represents the angle the track makes with the $z$-axis in the $x z$-plane.

The inner detector is made up of the Pixel Detector, the Semiconductor Tracker (SCT), and the Transition Radiation Tracker (TRT). Located at the centre of ATLAS the combination of these detectors provides tracking for charged particles resulting from the proton-proton collisions with pseudorapidity $|\eta|<2.5[5]$.

Moving outward, the inner detector is enclosed by a calorimetry system comprised of the Liquid Argon (LAr) calorimeters and the Tile calorimeter. Shown in orange in Figure 1.2, the LAr calorimeter is made up of an electromagnetic barrel calorimeter and two end-caps. Each end-cap has a LAr electromagnetic calorimeter followed by a hadronic calorimeter and the forward calorimeter in the high $\eta$ region. The electromagnetic calorimeter is designed to detect and measure the energy of incoming electrons and photons in the $|\eta|<1.5$ and $1.4<|\eta|<3.2$ region for the barrel and end-caps, respectively. The LAr hadronic calorimeter is set to detect and measure the energy of incoming hadrons (any matter made up of quarks) in the range of $1.5<|\eta|<3.2$. The LAr forward calorimeter is a combination of electromagnetic and hadronic calorimeters detecting particles in the $3.1<|\eta|<4.9$ range [6]. The Tile calorimeter is a hadronic calorimeter that surrounds the LAr barrel and endcaps providing further detection and measurement for high energy hadrons that pass through the LAr calorimeter with $|\eta|<1.7[7]$.

Since muons pass through these detectors mainly undetected, as the name suggests, the muon spectrometer is dedicated to reconstruct these particles. At large ra- 
dius, all particles, except neutrinos and muons, will be absorbed by the calorimeters. Hence the muon spectrometer subcomponents provide tracking for muons. Figure 1.3 depicts the Cathode Strip Chambers (CSC), Monitored Drift Tubes (MDT), Resistive Plate Chambers (RPC), and Thin Gap Chambers (TGC) that make up the muon spectrometer. Located at the centre of the Small Wheel (SW), the CSC is a Multiwire Proportional Chamber (MWPC) with segmented cathode strips that provide precise

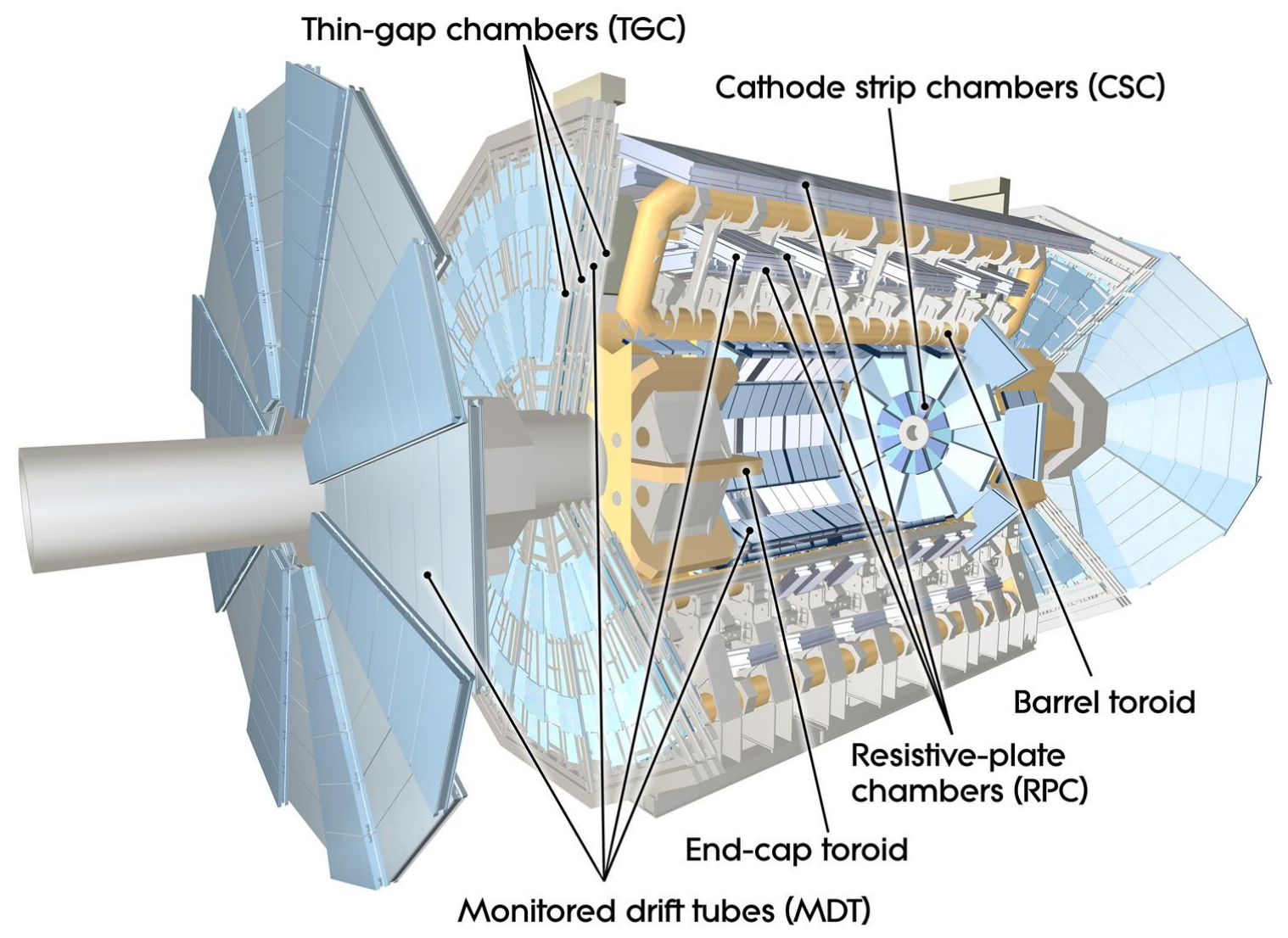

Figure 1.3: Illustration of the ATLAS muon spectrometer highlighting its subcomponents [8].

muon tracking in the high pseudorapidity region, $2.0<|\eta|<2.7$, of the ATLAS detector [9]. The MDT detectors are gas filled drift tubes located in both the barrel and end-cap regions of ATLAS to track muons with $|\eta|<2.7[10]$. The RPC is a gaseous detector that serves as the muon trigger in the barrel region [11]. Finally, the TGC's are MWPC's located on both the Big Wheel (BW) and SW used for triggering 
on muons in the end-cap regions [12]. Not labelled in Figure 1.3 are the TGC's that are part of the SW located in front of the end-cap toroid above the CSC's.

To prepare for the high luminosity conditions of the HL-LHC, the ATLAS muon spectrometer will undergo upgrades to its SW beginning in 2019. The SW will be replaced with the appropriately named New Small Wheel (NSW) to provide improved tracking and triggering for muons originating from the collision point within the detector.

\subsection{New Small Wheel (NSW)}

As mentioned previously, one of the primary objectives to be achieved by upgrading to the HL-LHC is to improve the understanding of the Higgs boson. One decay channel of the SM Higgs boson is via the Golden Channel (see Equation 1.2),

$$
H \rightarrow Z Z \rightarrow \ell^{+} \ell^{-} \ell^{+} \ell^{-}
$$

where $H$ represents the Higgs boson, $Z$ represents the $\mathrm{Z}$ boson, and $\ell^{-} / \ell^{+}$are any lepton/anti-lepton pair. The family of leptons consist of the electron, muon, tau, and their respective neutrinos. Since the $\mathrm{Z}$ boson is a neutral particle it can decay to an identical charged lepton/anti-lepton pair as to conserve both charge and lepton number. This can result in the production of two muon/anti-muon pairs from the Golden Channel decay process. Shown in Figure 1.4 is an event display of a Higgs boson to four muon candidate event. The four muons are signified by the red tracks originating from the IP and sections of the SW correspond to the green keystone shaped wedges illuminated in front of the end-cap toroid. The top-left corner of Figure 1.4 shows the number of interactions that occurred during the bunch crossing which highlights the difficulty of identifying the muons from the background. When the LHC enters the high luminosity phase the number of interactions will increase drastically thus significantly increasing the pile-up event rate. By effectively tracking muons that 


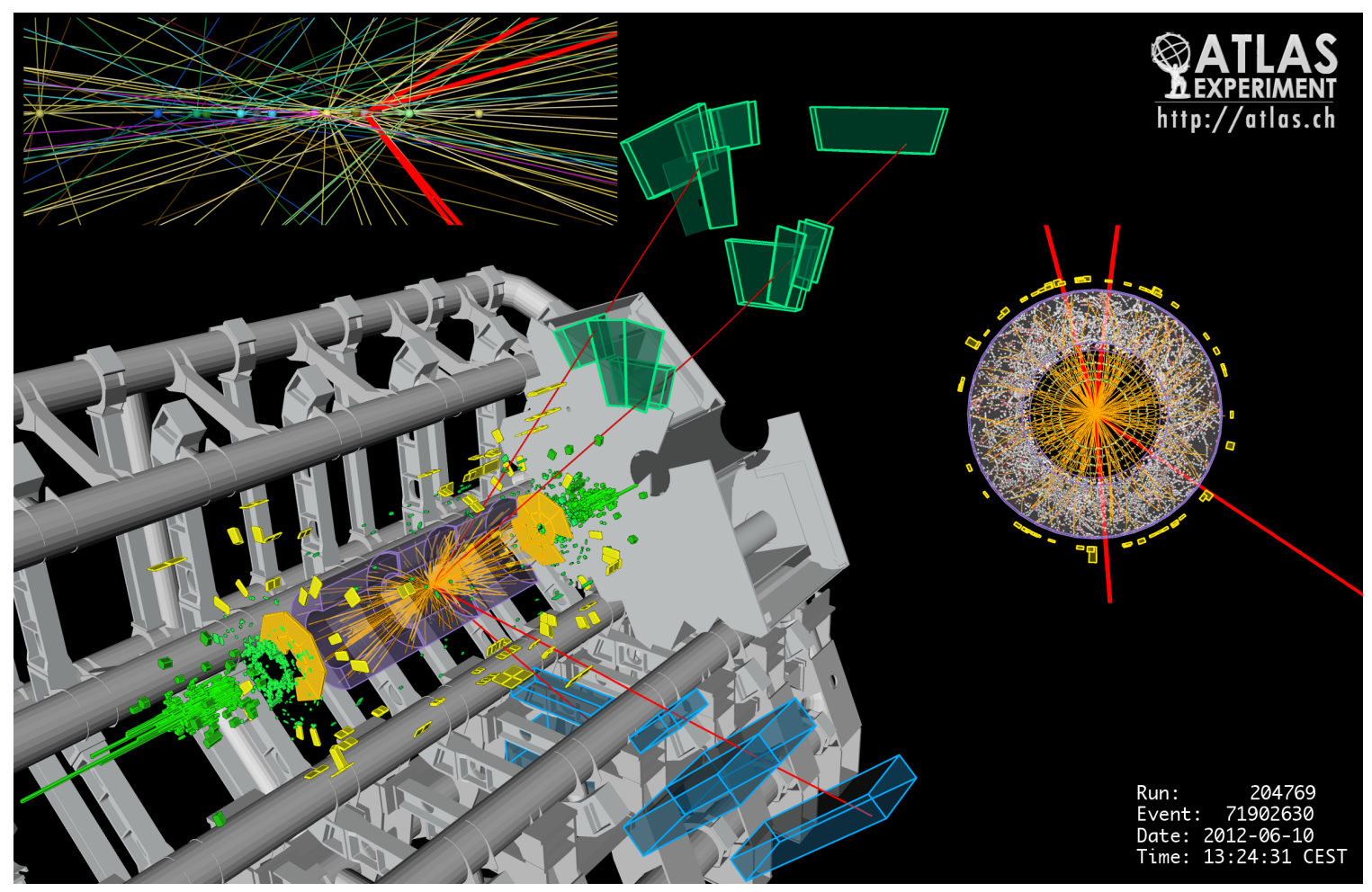

Figure 1.4: An event display showing a Higgs to four muon candidate event via the Golden Channel decay within the ATLAS detector. The red tracks correspond to the four muons originating from the IP. Sections of the SW are illuminated in green in front of the end-cap toroid signifying they detected the muon tracks. The top-left corner highlights the number of interactions which occurred during the bunch crossing and the middle-right shows all the charged particle tracks passing through ATLAS from the IP. [13]

originate from the IP, measurements can be made to further improve the study of the Higgs boson.

The NSW is the upcoming upgrade to the SW in the inner end-cap region of ATLAS. As shown in Figure 1.5, the NSW will be $9.3 \mathrm{~m}$ in diameter located $7.8 \mathrm{~m}$ along the $z$-axis from the IP [14]. The purpose of the NSW is to precisely reconstruct muons which originate from the IP. Furthermore, it serves as the Level-1 trigger in the inner end-cap to filter out muons that are falsely tagged by the BW to have originated from the IP. Presently, muon triggering is solely performed by the TGC detectors located in the BW. Referring to Figure 1.6, the BW can falsely tag muons 


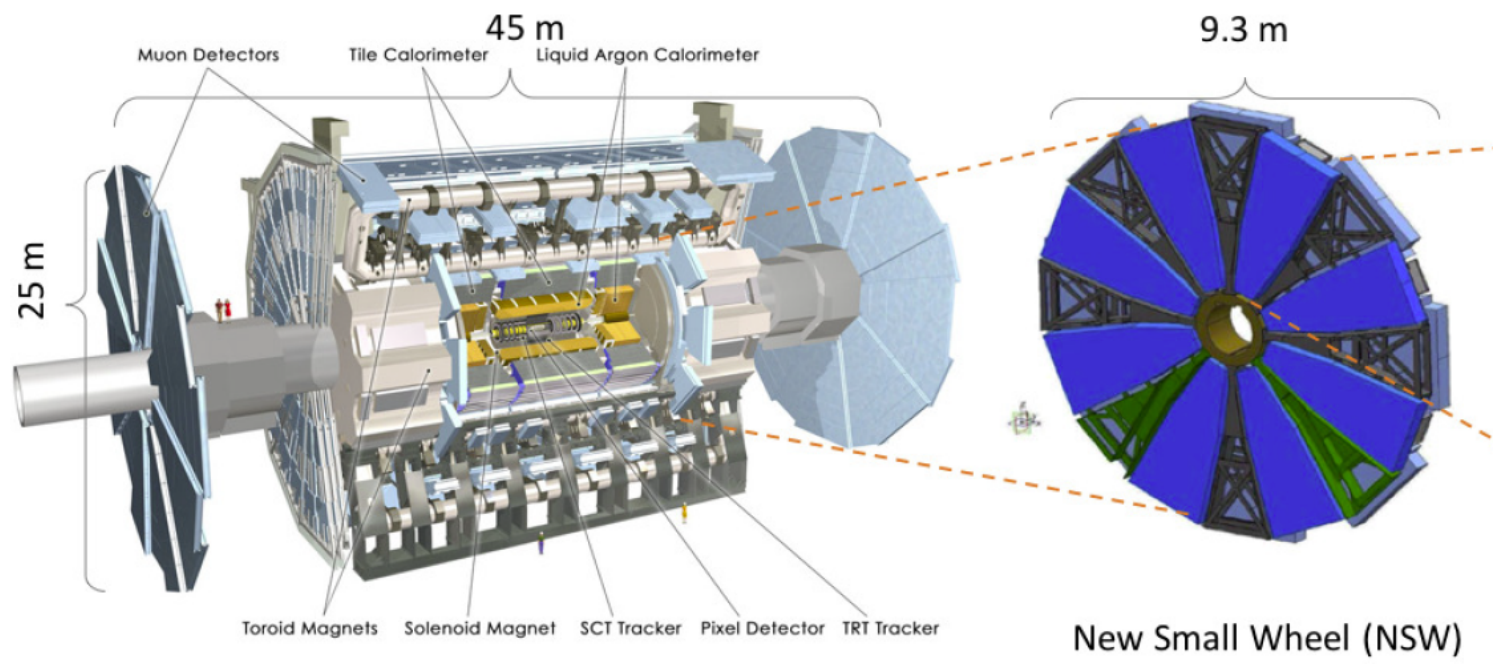

Figure 1.5: The location of the NSW in the inner end-cap region of the ATLAS detector [15].

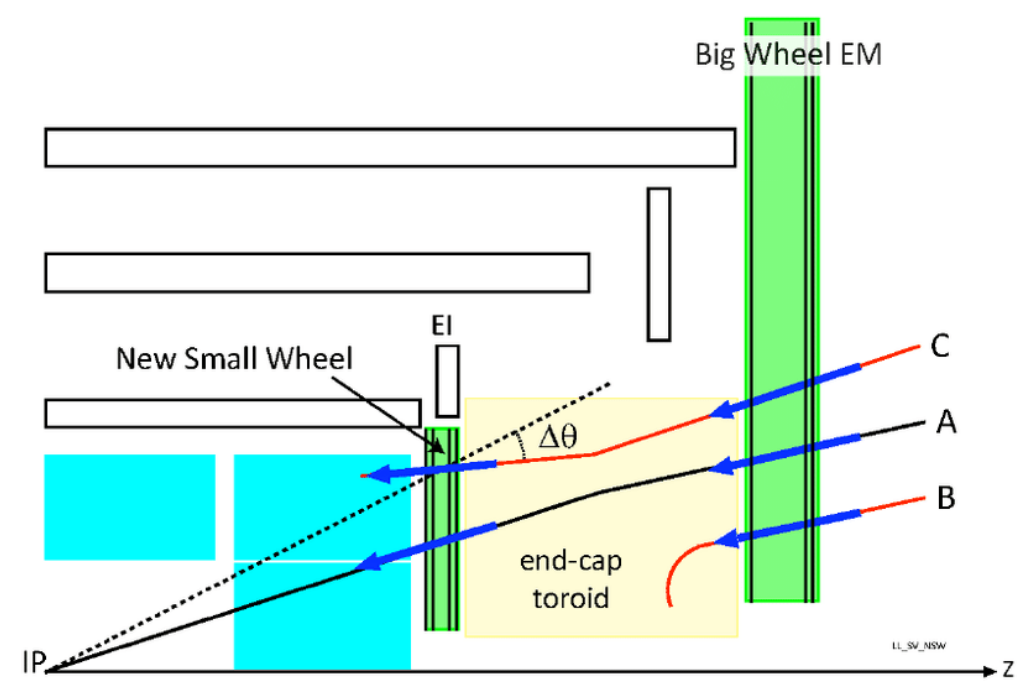

Figure 1.6: Trajectories of muons passing through the end-cap region of the ATLAS detector that have been tagged by the BW to have originated from IP. The installation of the NSW will allow the false muons for tracks B and C to be disregarded [16].

that have either undergone scattering within the detector or have had their trajectories bent due to the magnetic field so that they appear to originate from the IP [16].

As shown in Figure 1.7 the NSW is made up of 16 wedges, or sectors, and each wedge is made up of a sandwich of small-strip Thin Gap Chamber (sTGC) and 


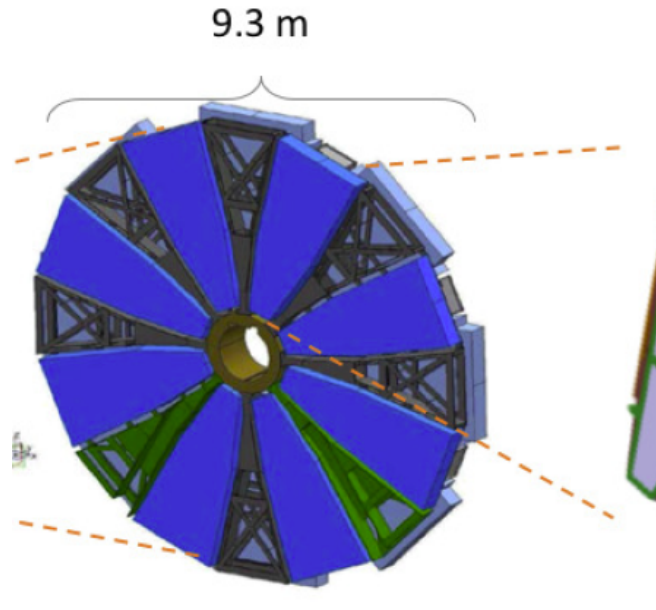

New Small Wheel (NSW) 2x 4 layers (Quadruplet) of Micromegas detectors / NSW sector

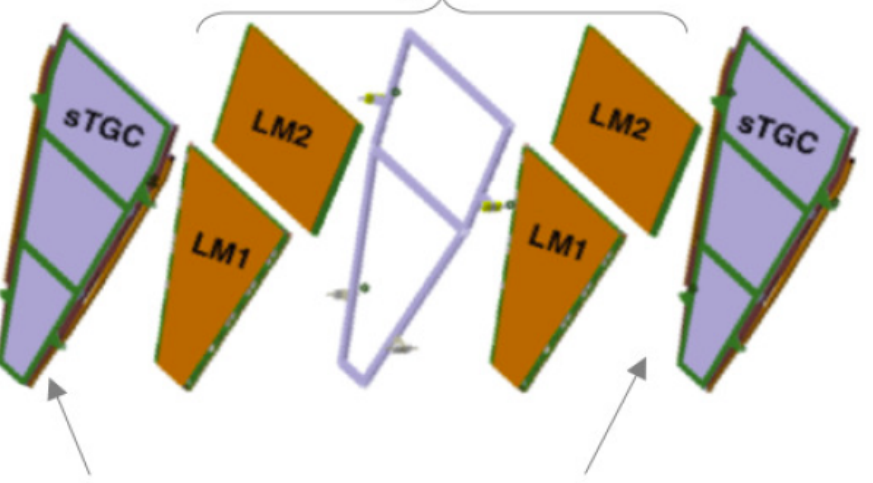

2x 4 layers (Quadruplet) of sTGC detectors / NSW sector

Figure 1.7: A NSW sector broken down into its four sub-layers. It is comprised of two inner MM layers each with four gas gaps of MM detectors sandwiched in between two sTGC outer layers each with four gas gaps of sTGC detectors [15].

Micromegas (MM) detectors. This is further illustrated in Figure 1.8, which shows the eight large sectors, eight small sectors, and the division of each into the three sTGC subsections on the outer layer. The small sectors and large sectors are keystone shaped and cover an angular range of $17^{\circ}$ and $28^{\circ}$, respectively, resulting in full $2 \pi$ coverage in $\phi$. In between the sTGC outer layers, the two MM layers are each comprised of four gaps of MM detector chambers. The MM is a gaseous particle detector that provides the precision tracking in the NSW for $1.3<|\eta|<2.7$ [14]. The outer layer is built with a quadruplet of sTGC detector chambers. Each sTGC quadruplet is an assembly of four gas gaps and is built in six different sizes. Referring to Figure 1.8, the outer layer is divided up into three subsections, labelling each sector from smallest to largest these are: QS1, QS2, QS3, QL1, QL2, and QL3. Where the label Q represents the quadruplet, S and L correspond to small and large sector, respectively, and 1, 2, and 3 define the sections increasing in size. The sTGC increase in size radially to allow full $2 \pi$ coverage over the NSW. The sTGC is a MWPC gaseous detector used to provide triggering and tracking on muons originating from the IP with $1.3<|\eta|<2.5$. The 


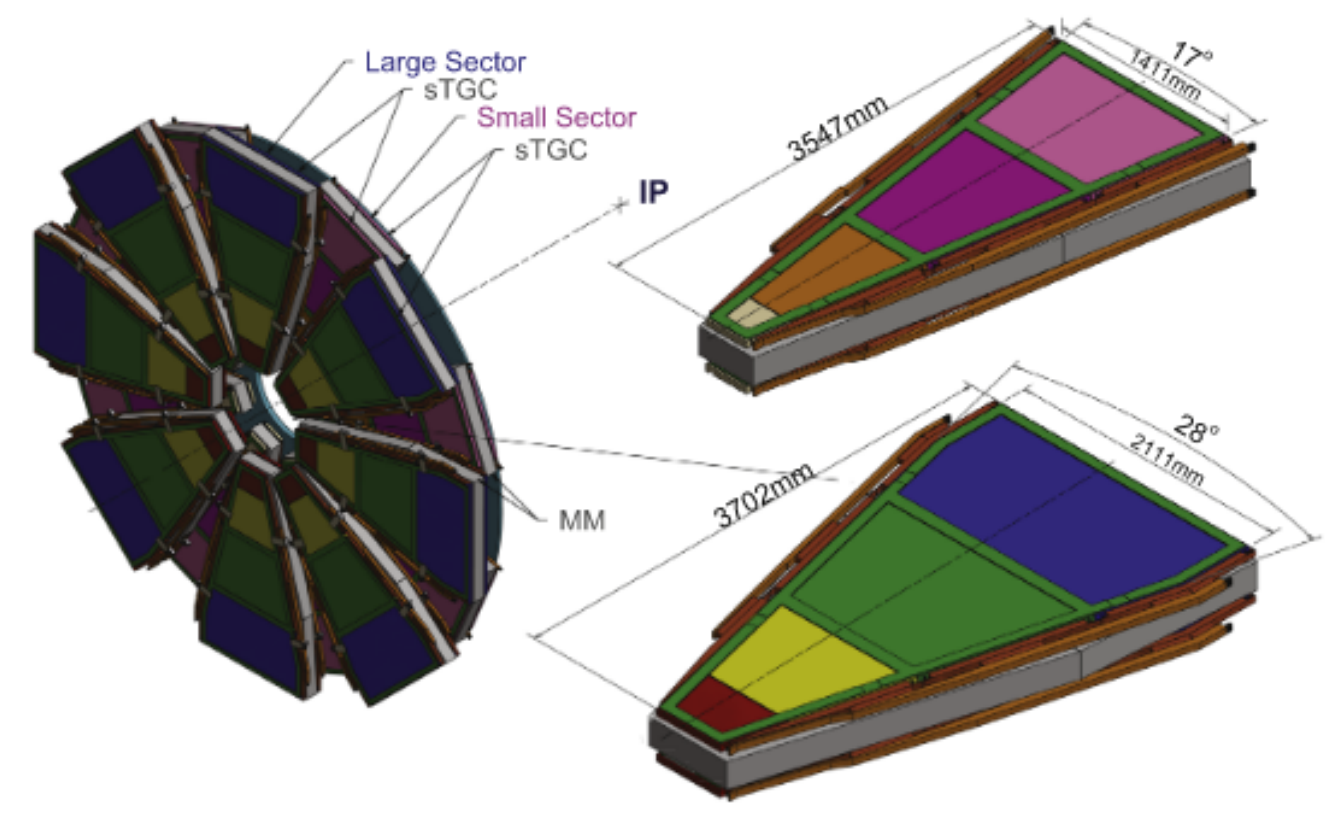

Figure 1.8: NSW Small and Large Sectors. The three subsections of each sector signify the three sTGC systems. For the small sector, the orange/beige is QS1, magenta is QS2, and pink is QS3. For the large sector, the red/yellow is QL1, the green is QL2, and the blue is QL3.

beige and red tips of the QS1 and QL1, respectively, in Figure 1.8 highlight the section of the quadruplet's where the signals from the wires are not read out due to events occurring at too great of a rate to be recorded. A further description of the sTGC detection, triggering, and tracking techniques will be provided in Chapter 4 .

The ATLAS team at Carleton University are currently working on the assembly and testing of the QS3 and QL2 sTGC quadruplets which will be part of the upgrade to the ATLAS muon spectrometer. The work presented in this thesis will focus on the simulation of a single sTGC gas gap.

\subsection{Motivation}

With the upcoming upgrade to the ATLAS NSW a method to successfully simulate the sTGC detector becomes increasingly important for the deployment of the newly built detectors. The sTGC is a resistive segmented cathode MWPC with read- 
out electronics that monitor for signal above a set threshold. As a charged particle passes through the detection chamber it ionizes the gas inside and the drifting of this ionized electron and ion will induce charge on the segmented electrodes. This thesis aims to show that by considering the factors that lead to signal formation, gaseous detectors can be simulated using Ramo's theorem. Moving ahead, this idea plans to be built upon to determine a method to improve hit clustering and two-track separation resolution for two particles passing through the sTGC. As highlighted above, a Higgs boson can result in two muon/anti-muon pairs but if these pairs are too close the detector will not be able to resolve them.

\subsection{Thesis Layout}

In this thesis, data from the October 2017 sTGC test beam at CERN is compared to simulation results. The sTGC detector is simulated and the effects on signal formation are carried out. The results from 1000 simulations involving a single muon track were analyzed and averaged to compare to the test beam data.

The thesis proceeds as follows:

1. Chapter 2 begins with the derivation of the reciprocity theorem to illustrate the relationship between two separate systems of conductors. This is followed by explaining how charge is induced on segmented electrodes due to a nearby stationary point charge using Ramo's theorem. Next, this concept is expanded to the case of the total induced charge due to a moving point charge in a system of segmented electrodes.

2. Chapter 3 highlights the fundamental physics of a resistive cathode MWPC. A description of the processes of ionization and charge cluster transport in a gaseous charged particle detector will be given. It will then go on to explain 
how charge disperses over a resistive layer with symmetric ground, leading into charge dispersion on a layer grounded on one side.

3. Chapter 4 starts with an overview of the sTGC detector. This leads into the method of simulating the signals produced in the sTGC. An explanation of how signal is produced on the wires as well the determination of the time of arrival of electron clusters to the wire is given. Continuing, the detector response to the readout electronics is described as well as the procedure to account for it in the simulation. The chapter concludes with an illustration of the signal production on the segmented cathode strips with a resistive layer, finalizing with the steps to model it in the signal processing.

4. Chapter 5 leads off with the logic behind the decision to appropriately fit the charge clusters to reconstruct the $y$-coordinate of the simulated muon track. This is followed by the an explanation of the two techniques that can be used to determine the centre of a charge cluster. Finally, the results of the track reconstruction will be presented.

5. Chapter 6 begins by outlining the procedure to compare the simulated results to test beam data. From this a method for determining the appropriate $R C$ time constant to be used in the simulations is given. To conclude, the results of the analysis are presented to give a value for the time constant.

Overall, the work summarized here is meant to tune the simulation of gas detectors using Ramo's theorem in order to further develop the ability to design and construct the next generation of instrumentation for future experiments in high energy physics. The simulation framework presented in this thesis was originally developed for the foreseen Time Projection Chamber (TPC) for the International Linear Collider (ILC), and it is applied here to the ATLAS sTGC. 


\section{Chapter 2}

\section{Charge Induction and Ramo's}

\section{Theorem}

\section{$2.1 \quad$ Reciprocity Theorem}

The reciprocity theorem relates two separate systems and can be used to determine the charge or potential of one system provided information is known about the other.

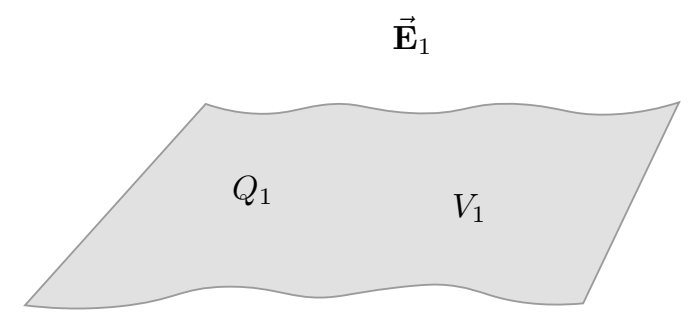

(a)

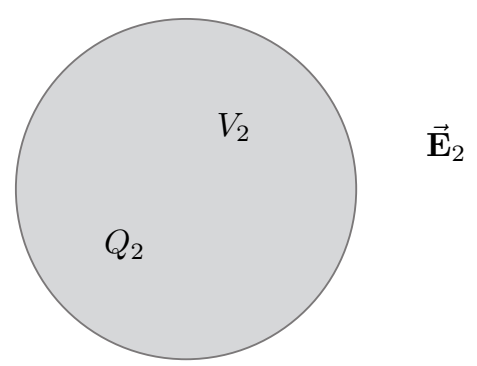

(b)

Figure 2.1: (a) A conducting surface carrying charge $Q_{1}$ with potential $V_{1}$ producing an electric field $\overrightarrow{\mathbf{E}}_{1}$ in the space surrounding it. (b) A separate circular conducting surface carrying charge $Q_{2}$ with potential $V_{2}$ that produces a different electric field $\overrightarrow{\mathbf{E}}_{2}$ in its surrounding area. 
Consider the two independent conducting surfaces illustrated in Figure 2.1a and 2.1b, each carries a charge $Q_{i}$ with the surface at some potential $V_{i}$ to produce an electric field $\overrightarrow{\mathbf{E}}_{i}$. The two systems can be related using the reciprocity theorem by first solving

$$
\int_{\text {all space }} \overrightarrow{\mathbf{E}}_{1} \cdot \overrightarrow{\mathbf{E}}_{2} d \tau
$$

Using the fact any electric field is related to its potential by $\overrightarrow{\mathbf{E}}=-\vec{\nabla} V$, this is subbed into Equation 2.1 giving

$$
\int_{\text {all space }} \overrightarrow{\mathbf{E}}_{1} \cdot \overrightarrow{\mathbf{E}}_{2} d \tau=\int_{\text {all space }} \overrightarrow{\mathbf{E}}_{1} \cdot\left(-\vec{\nabla} V_{2}\right) d \tau
$$

The right-hand side of Equation 2.2 is rearranged using the vector identity

$$
\vec{\nabla} \cdot(f \overrightarrow{\mathbf{A}})=f(\vec{\nabla} \cdot \overrightarrow{\mathbf{A}})+\overrightarrow{\mathbf{A}} \cdot \vec{\nabla} f
$$

where $f$ is some scalar and $\overrightarrow{\mathbf{A}}$ is any vector, which results in

$$
\int_{\text {all space }} \overrightarrow{\mathbf{E}}_{1} \cdot \overrightarrow{\mathbf{E}}_{2} d \tau=\int_{\text {all space }}\left[V_{2}\left(\vec{\nabla} \cdot \overrightarrow{\mathbf{E}}_{1}\right)-\vec{\nabla} \cdot\left(V_{2} \overrightarrow{\mathbf{E}}_{1}\right)\right] d \tau
$$

The first term on the right side of Equation 2.4 is rewritten using the Maxwell relation $\vec{\nabla} \cdot \overrightarrow{\mathbf{E}}_{1}=\rho_{1} / \epsilon_{0}$ and the second term using the relation of the electric field and the potential stated above

$$
\int_{\text {all space }} \overrightarrow{\mathbf{E}}_{1} \cdot \overrightarrow{\mathbf{E}}_{2} d \tau=\int_{\text {all space }}\left[\frac{\rho_{1} V_{2}}{\epsilon_{0}}-\vec{\nabla} \cdot\left(V_{2} \vec{\nabla} V_{1}\right)\right] d \tau .
$$

The second term in Equation 2.5 was again simplified using another vector identity

$$
\int(\vec{\nabla} \cdot \overrightarrow{\mathbf{A}}) d \tau=\oint \overrightarrow{\mathbf{A}} \cdot \overrightarrow{\mathbf{d a}}
$$


This substitution to Equation 2.5 results in

$$
\int_{\text {all space }} \overrightarrow{\mathbf{E}}_{1} \cdot \overrightarrow{\mathbf{E}}_{2} d \tau=\int_{\text {all space }} \frac{\rho_{1} V_{2}}{\epsilon_{0}} d \tau-\oint_{\text {all space }}\left(V_{2} \vec{\nabla} V_{1}\right) \cdot \overrightarrow{\mathbf{d a}} .
$$

The scalar potentials of the two surfaces in Figure 2.1 are chosen such that they are zero infinitely far away. This results in the second integral in Equation 2.7 to be zero giving the final solution to be

$$
\int_{\text {all space }} \overrightarrow{\mathbf{E}}_{1} \cdot \overrightarrow{\mathbf{E}}_{2} d \tau=\int_{\text {all space }} \frac{\rho_{1} V_{2}}{\epsilon_{0}} d \tau .
$$

Choosing to sub in for $\overrightarrow{\mathbf{E}}_{2}$ in Equation 2.2 is completely arbitrary and $\overrightarrow{\mathbf{E}}_{1}$ can just as easily be subbed into. Subbing in for $\overrightarrow{\mathbf{E}}_{1}$ following the procedure above yields

$$
\int_{\text {all space }} \overrightarrow{\mathbf{E}}_{1} \cdot \overrightarrow{\mathbf{E}}_{2} d \tau=\int_{\text {all space }} \frac{\rho_{2} V_{1}}{\epsilon_{0}} d \tau .
$$

Equating Equation 2.8 and Equation 2.9 produces

$$
\int_{\text {all space }} \frac{\rho_{1} V_{2}}{\epsilon_{0}} d \tau=\int_{\text {all space }} \frac{\rho_{2} V_{1}}{\epsilon_{0}} d \tau,
$$

from which both integrals can be evaluated to give

$$
Q_{1} V_{2}=Q_{2} V_{1}
$$

The final result shown Equation 2.11 is the reciprocity theorem and it illustrates how two independent systems can be related to determine the charge or potential of one surface provided information about the other is known.

The reciprocity theorem can be extended to a system that contains $n$ electrodes. For a given configuration of $n$ conductors each with potential $V_{i}$ and charge $Q_{i}$, the dependence of the potential on charge density allows the potential of the $i^{\text {th }}$ conductor 
to be written as $[17]$

$$
V_{i}=\sum_{j=1}^{n} p_{i j} Q_{j},
$$

where $p_{i j}$ depends on the geometry of the conductors. The potential and charge of each conductor are combined into respective vectors

$$
\vec{V}=\left[\begin{array}{c}
V_{1} \\
V_{2} \\
\vdots \\
V_{n}
\end{array}\right], \quad \vec{Q}=\left[\begin{array}{c}
Q_{1} \\
Q_{2} \\
\vdots \\
Q_{n}
\end{array}\right],
$$

allowing Equation 2.12 to be re-written as

$$
\vec{V}=P \vec{Q},
$$

where $P$ is the matrix containing the $p_{i j}$ coefficients. Equation 2.13 can be inverted to give

$$
\vec{Q}=P^{-1} \vec{V},
$$

where $P^{-1}=C$ is the capacitance matrix of the system. The matrix elements of $C$, $c_{i i}$ and $c_{i j}$, are the capacitances and coefficients of induction, respectively [17]. The coefficients of induction have the property that $c_{i j}=c_{j i}$ since the charge induced on electrode $i$ by $j$ will be equivalent to the charge induced on $j$ by $i$. Equation 2.14 is re-written as

$$
\vec{Q}=C \vec{V},
$$

to relate the charge and potentials of $n$ electrodes within a system. Now consider a system with identical geometry to the system described by Equation 2.15 but with 
charges $\bar{Q}_{i}$ and potentials $\bar{V}_{i}$ to give

$$
\overrightarrow{\bar{Q}}=C^{\prime} \vec{V} .
$$

Since the geometry of the systems described by Equation 2.15 and Equation 2.16 are identical, it can be argued that $C^{\prime}=C$ because $C=P^{-1}$ where $P$ depends on the geometry of the conductors. The dot product of the charge vector from the system represented in Equation 2.15 with the vector of potentials from the system in Equation 2.16 produces

$$
\begin{gathered}
\vec{Q} \cdot \vec{V}=\vec{Q}^{T} \vec{V} \\
\vec{Q} \cdot \overrightarrow{\vec{V}}=(C \vec{V})^{T} \vec{V}, \\
\vec{Q} \cdot \overrightarrow{\vec{V}}=\vec{V}^{T} C^{T} \vec{V}
\end{gathered}
$$

It was previously presented that $c_{i j}=c_{j i}$, therefore inferring that $C^{T}=C$ in Equation 2.17 which gives

$$
\begin{gathered}
\vec{Q} \cdot \vec{V}=\vec{V}^{T}(C \overrightarrow{\bar{V}}), \\
\vec{Q} \cdot \vec{V}=\vec{V}^{T} \vec{Q}, \\
\vec{Q} \cdot \vec{V}=\vec{V}^{T} \vec{Q} \\
\vec{Q} \cdot \vec{V}=\vec{V} \cdot \overrightarrow{\bar{Q}} .
\end{gathered}
$$

By evaluating the dot product on both sides of Equation 2.18 results in the sum of the products of each element

$$
\sum_{i=1}^{n} Q_{i} \bar{V}_{i}=\sum_{i=1}^{n} V_{i} \bar{Q}_{i} .
$$

The result presented in Equation 2.19 is analogous to the solution shown in Equation 2.11 and is the reciprocity theorem for a system of $n$ electrodes. As will be explained in the subsequent section, this version of the reciprocity theorem is the 
basis of charge induction using Ramo's theorem and allows for the determination of charge induced on electrodes due to a nearby charge.

\subsection{Stationary Charge}

The determination of the charge induced on a single grounded conductor by a nearby point charge is a trivial problem, however, in the situation where the single conductor is replaced with several segmented electrodes, solving the charge induced on an individual surface is no longer a simple task. Ramo's theorem provides an intuitive method to determine the charge induced on an individual conductor by the nearby point charge. The basis of Ramo's theorem relates two separate systems using the reciprocity theorem as explained previously. Consider the system illustrated in Figure $2.2 \mathrm{a}$, a stationary point charge is in the area near four grounded conductors. To determine the charge induced on each electrode using the reciprocity theorem, the point charge is assumed to rest on an infinitesimal electrode with a potential $V_{0}$ due to the point charge. Figure $2.2 \mathrm{~b}$ depicts an identical system to $2.2 \mathrm{a}$ but the charge has been removed and electrode 1 is raised to a potential $V_{w}$. Setting the electrode to this potential produces a weighted potential in the surrounding area, with $\psi_{1}(x)$ representing the value of the weighted potential at the location where the point charge is in the original system. The two systems in Figure 2.2 are related using Equation 2.19 yielding

$$
Q_{0} \bar{V}_{0}+Q_{1} \bar{V}_{1}+Q_{2} \bar{V}_{2}+Q_{3} \bar{V}_{3}+Q_{4} \bar{V}_{4}=V_{0} \bar{Q}_{0}+V_{1} \bar{Q}_{1}+V_{2} \bar{Q}_{2}+V_{3} \bar{Q}_{3}+V_{4} \bar{Q}_{4}
$$

The respective values of the charge and potential are subbed in

$$
q\left(\psi_{1}(x)\right)+Q_{1}\left(V_{w}\right)+Q_{2}(0)+Q_{3}(0)+Q_{4}(0)=V_{0}(0)+(0) \bar{Q}_{1}+(0) \bar{Q}_{2}+(0) \bar{Q}_{3}+(0) \bar{Q}_{4},
$$



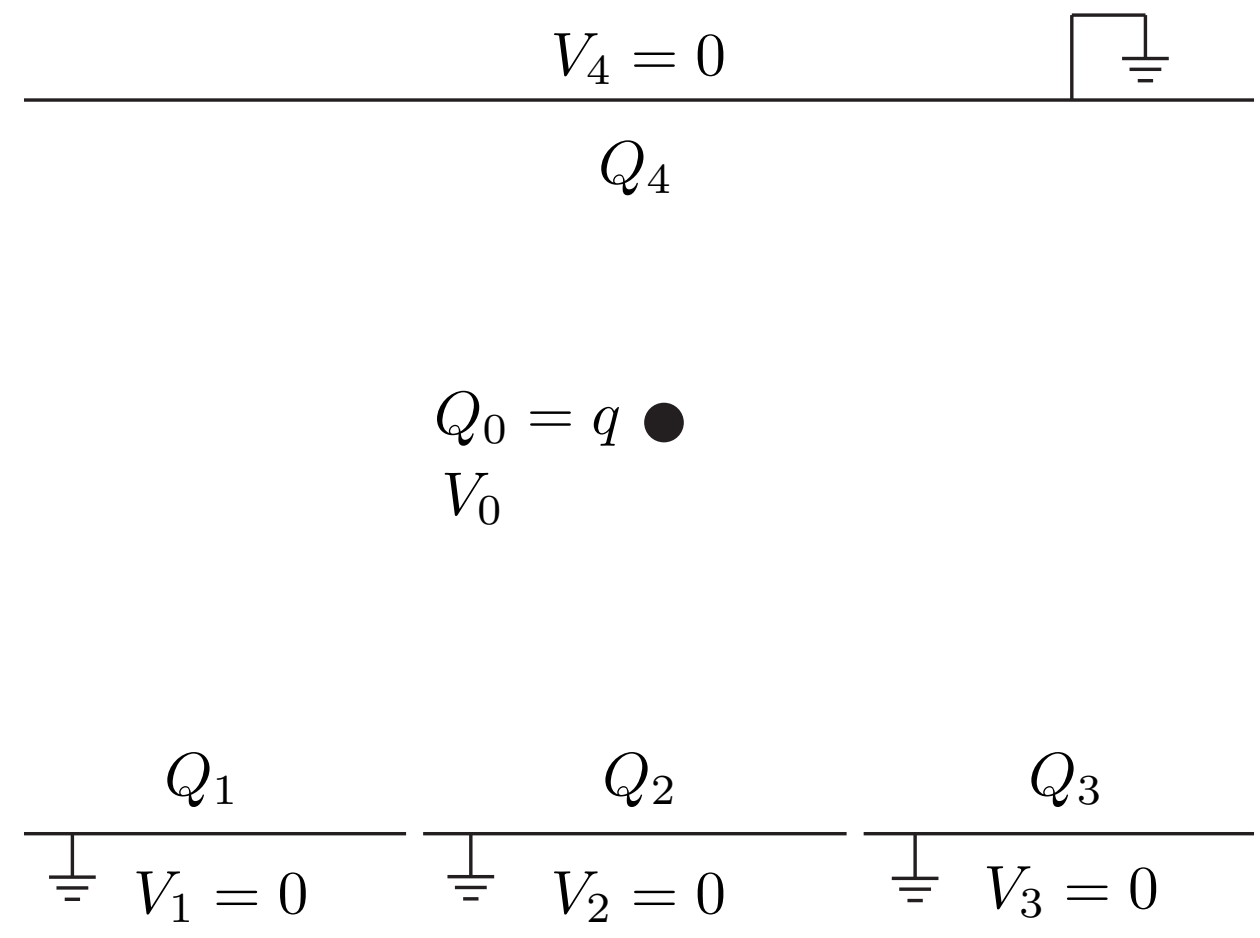

(a)

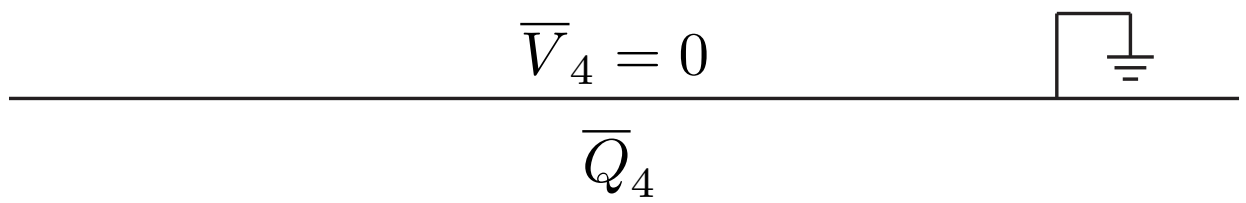

$$
\begin{aligned}
& \bar{Q}_{0}=0 \\
& \bar{V}_{0}=\psi_{1}(x)
\end{aligned}
$$

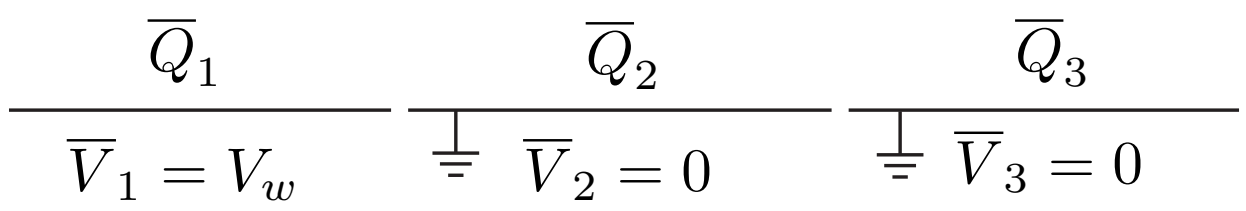

(b)

Figure 2.2: (a) A point charge in the area of four grounded conductors. Each electrode has some induced charge $Q_{i}$ due to the point charge $q$. (b) An identical system to (a) with the point charge removed and electrode 1 raised to potential $V_{w}$. 


$$
\begin{gathered}
q \psi_{1}(x)+Q_{1} V_{w}=0, \\
Q_{1} V_{w}=-q \psi_{1}(x), \\
Q_{1}=-q \frac{\psi_{1}(x)}{V_{w}} .
\end{gathered}
$$

Equation 2.21 is the total charge, $Q_{1}$, that is inducted on electrode 1 due to the point charge $q$. The total charge is proportional to the ratio of the weighted field, $\psi_{1}(x)$, at the location of the point charge with the potential, $V_{w}$, electrode 1 is set to in the alternate system.

To solve for charges induced on electrodes 2, 3, and 4 the alternate systems shown in Figure 2.3a, b, and c, respectively, are used. By using the conditions outlined in Figure 2.3a, Equation 2.20 is rewritten as

$$
\begin{gathered}
q\left(\psi_{2}(x)\right)+Q_{1}(0)+Q_{2}\left(V_{w}\right)+Q_{3}(0)+Q_{4}(0)=V_{0}(0)+(0) \bar{Q}_{1}+(0) \bar{Q}_{2}+(0) \bar{Q}_{3}+(0) \bar{Q}_{4} \\
q \psi_{2}(x)+Q_{2} V_{w}=0 \\
Q_{2} V_{w}=-q \psi_{2}(x) \\
Q_{2}=-q \frac{\psi_{2}(x)}{V_{w}}
\end{gathered}
$$

where $\psi_{2}(x)$ is the weighted potential produced by setting electrode 2 to $V_{w}$ in the alternate system. Similarly, Equation 2.20 is modified according to Figure 2.3b to give

$$
\begin{gathered}
q\left(\psi_{3}(x)\right)+Q_{1}(0)+Q_{2}(0)+Q_{3}\left(V_{w}\right)+Q_{4}(0)=V_{0}(0)+(0) \bar{Q}_{1}+(0) \bar{Q}_{2}+(0) \bar{Q}_{3}+(0) \bar{Q}_{4}, \\
q \psi_{3}(x)+Q_{3} V_{w}=0 \\
Q_{3} V_{w}=-q \psi_{3}(x)
\end{gathered}
$$




\begin{tabular}{|c|c|c|}
\hline & $\bar{V}_{4}=0$ & $\stackrel{1}{\equiv}$ \\
\hline \multicolumn{3}{|c|}{$\bar{Q}_{4}$} \\
\hline \multicolumn{3}{|c|}{$\begin{array}{l}\bar{Q}_{0}=0 \\
\bar{V}_{0}=\psi_{2}(x)\end{array}$} \\
\hline $\bar{Q}_{1}$ & $\bar{Q}_{2}$ & $\bar{Q}_{3}$ \\
\hline$\stackrel{\perp}{=} \bar{V}_{1}=0$ & $\bar{V}_{2}=V_{w}$ & $\stackrel{\perp}{=} \bar{V}_{3}=0$ \\
\hline
\end{tabular}

(a)

\begin{tabular}{c|c}
$\bar{V}_{4}=0$ & $\square$ \\
\hline $\bar{Q}_{4}$ &
\end{tabular}

$$
\begin{aligned}
& \bar{Q}_{0}=0 \\
& \bar{V}_{0}=\psi_{3}(x)
\end{aligned}
$$

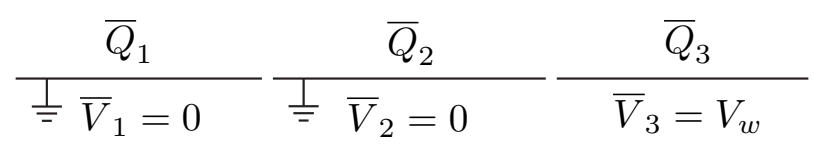

(b)

$\frac{\bar{V}_{4}=V_{w}}{\bar{Q}_{4}}$

$$
\begin{aligned}
& \bar{Q}_{0}=0 \\
& \bar{V}_{0}=\psi_{4}(x)
\end{aligned}
$$

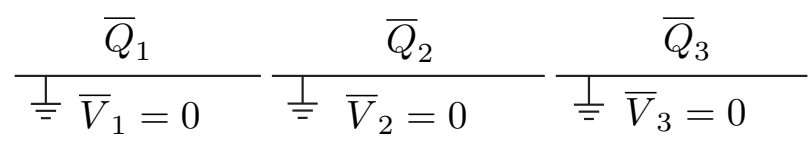

(c)

Figure 2.3: (a) The alternate system to determine charge induced on electrode 2 setting it to $V_{w}$. (b) The alternate system used in determining charge induced on electrode 3. (c) The system used to determine the charge induced on electrode 4. 


$$
Q_{3}=-q \frac{\psi_{3}(x)}{V_{w}},
$$

with $\psi_{3}(x)$ being the weighted field produced by electrode 3. Finally, Equation 2.20 is evaluated using the conditions from Figure 2.2a and Figure 2.3d to determine the charge induced on electrode 4 . The result of this gives

$$
\begin{gathered}
q\left(\psi_{4}(x)\right)+Q_{1}(0)+Q_{2}(0)+Q_{3}(0)+Q_{4}\left(V_{w}\right)=V_{0}(0)+(0) \bar{Q}_{1}+(0) \bar{Q}_{2}+(0) \bar{Q}_{3}+(0) \bar{Q}_{4}, \\
q \psi_{4}(x)+Q_{4} V_{w}=0 \\
Q_{4} V_{w}=-q \psi_{4}(x) \\
Q_{4}=-q \frac{\psi_{4}(x)}{V_{w}}
\end{gathered}
$$

where $\psi_{4}(x)$ is the weighted potential due to electrode 4 .

From Equations 2.21, 2.22, 2.23, and 2.24 the charge induced on all conductors in the original system are able to be determined by modelling a similar system where the point charge is removed. This outcome can be generalized to [18]

$$
Q_{i}^{\text {ind }}=-q \frac{\psi_{i}(x)}{V_{w}},
$$

with $Q_{i}^{\text {ind }}$ being the charge induced on electrode $i$ due to the point charge $q$. By considering the alternate system, $V_{w}$ is the potential electrode $i$ is set to which produces a weighted potential $\psi_{i}(x)$ at the location the point charge is removed from. The generalized conclusion of Equation 2.25 is Ramo's theorem for a stationary point charge which states that the total induced charge on an electrode is equivalent to the negative of the point charge scaled by the ratio of the weighted potential with the potential the electrode is set to in the alternate system. 


\subsection{Moving Charge}

The case of a stationary point charge is great to introduce the fundamentals of charge induction using Ramo's theorem, but it does not provide a proper representation of the physics that occurs in a gaseous detector. This is better described by studying the charge that is induced on a grounded electrode due to a moving point charge. In Figure $2.4 \mathrm{a}$ a point charge moves with some velocity $\vec{v}$ from $x_{0}$ to $x_{1}$ in between two grounded conducting planes. The motion of the point charge induces an electrical current to flow from the electrode to ground. By considering the system in Figure $2.4 \mathrm{~b}$, the current that flows through electrode 2 is determined by the weighted potential produced by raising the electrode to the potential $V_{w}$. Since current is the change in charge with respect to time, the current that is induced through electrode 2 is determined by taking the time derivative of Equation 2.22

$$
\begin{gathered}
-\frac{d}{d t}\left(Q_{2}\right)=-\frac{d}{d t}\left(-q \frac{\psi_{2}(x)}{V_{w}}\right), \\
I_{2}^{i n d}=\frac{q}{V_{w}} \frac{d\left(\psi_{2}(x)\right)}{d t}, \\
I_{2}^{i n d}=\frac{q}{V_{w}} \nabla \psi_{2}(x) \cdot \frac{d \vec{x}}{d t} \\
I_{2}^{i n d}=-\frac{q}{V_{w}} \vec{E}_{2}[x(t)] \cdot \vec{v}(t) .
\end{gathered}
$$

The definition of $I_{2}=-d Q_{2} / d t$ indicates that current that flows away from the electrode to ground is taken as positive. In Equation 2.26, $\vec{v}(t)$ is the velocity the charged particle traverses the region with and $\vec{E}_{2}[x(t)]$ is the electric field produced in Figure 2.4b when electrode 2 is raised to $V_{w}$. This electric field is referred to as the weighted field produced by electrode 2 since it is related to the weighted potential by 


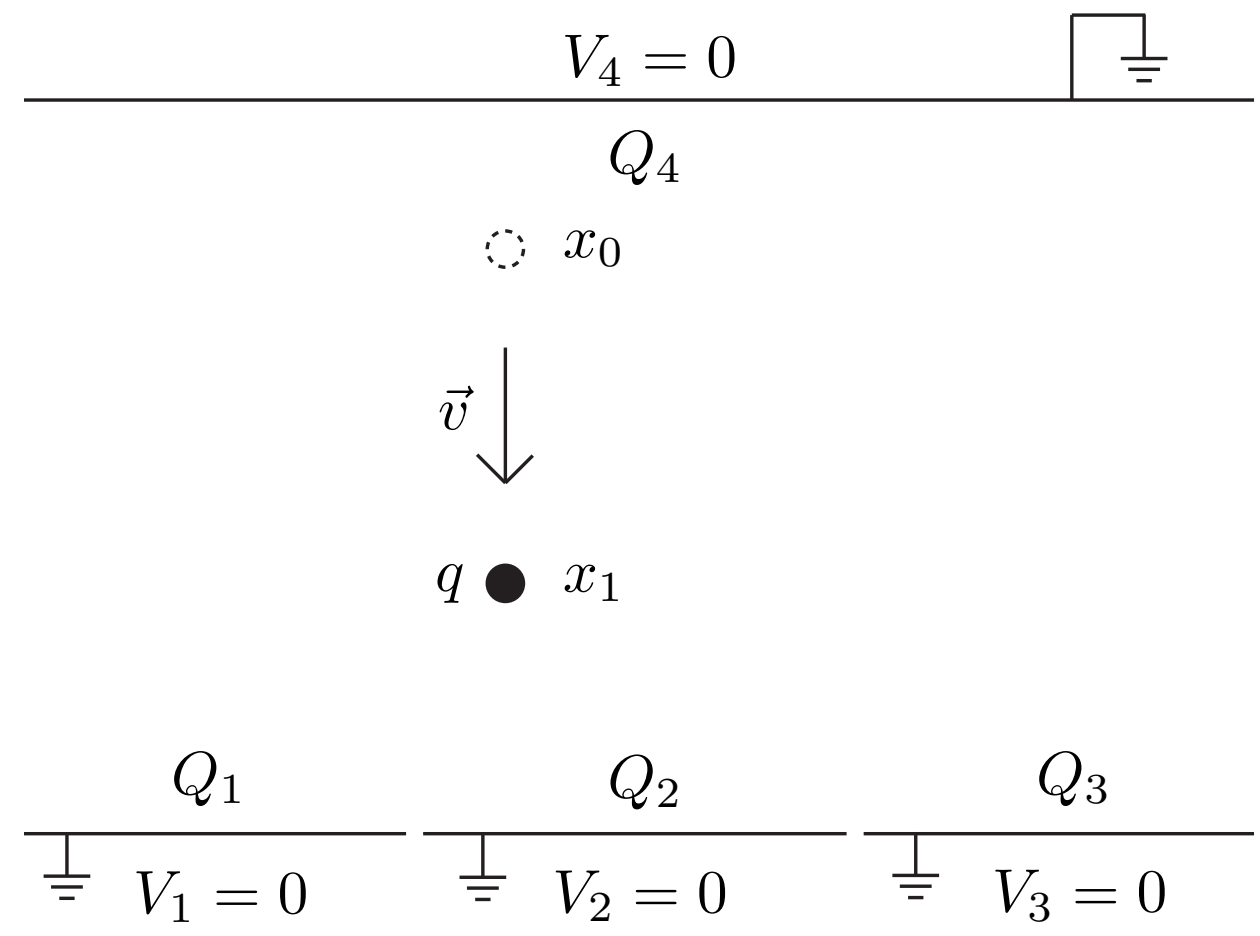

(a)

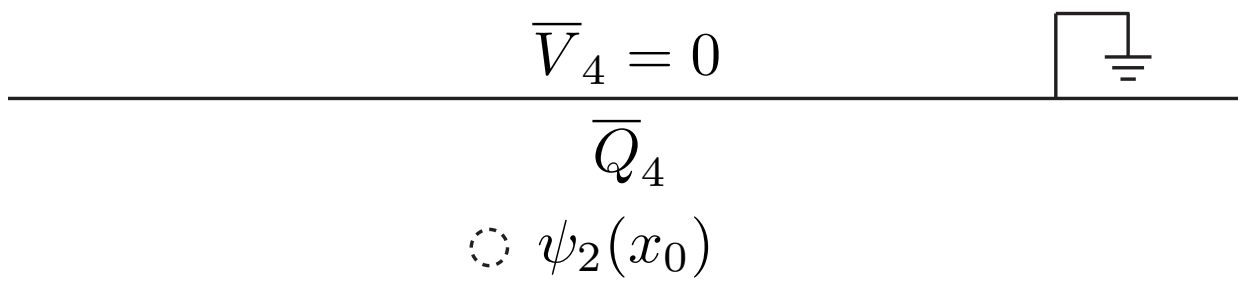

$$
\therefore \psi_{2}\left(x_{1}\right)
$$

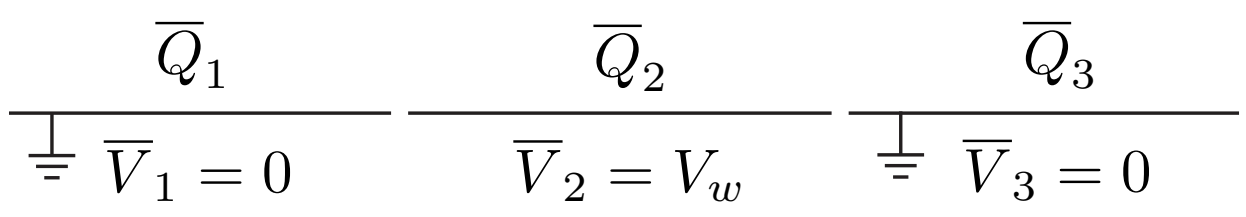

(b)

Figure 2.4: (a) A charged particle $q$ moves from $x_{0}$ to $x_{1}$ with velocity $\vec{v}$ in between 2 electrode planes. (b) The alternate system where the point charge has been removed. Electrode 2 is set to $V_{w}$ producing a weighted potential given by $\psi_{2}(x)$. The initial and final locations of the point charge are highlighted in the weighted potential. 
$\vec{E}_{2}=-\nabla \psi_{2}(x)$. The weighted field produced when $\bar{V}_{2}$ is set to $V_{w}=1 \mathrm{~V}$ is illustrated in Figure 2.5 which highlights the equipotential lines of the field. The sign of the current depends both on if the moving point charge is positive or negative as well as the direction the velocity vector makes with the weighted field that is produced in the alternate configuration [18].

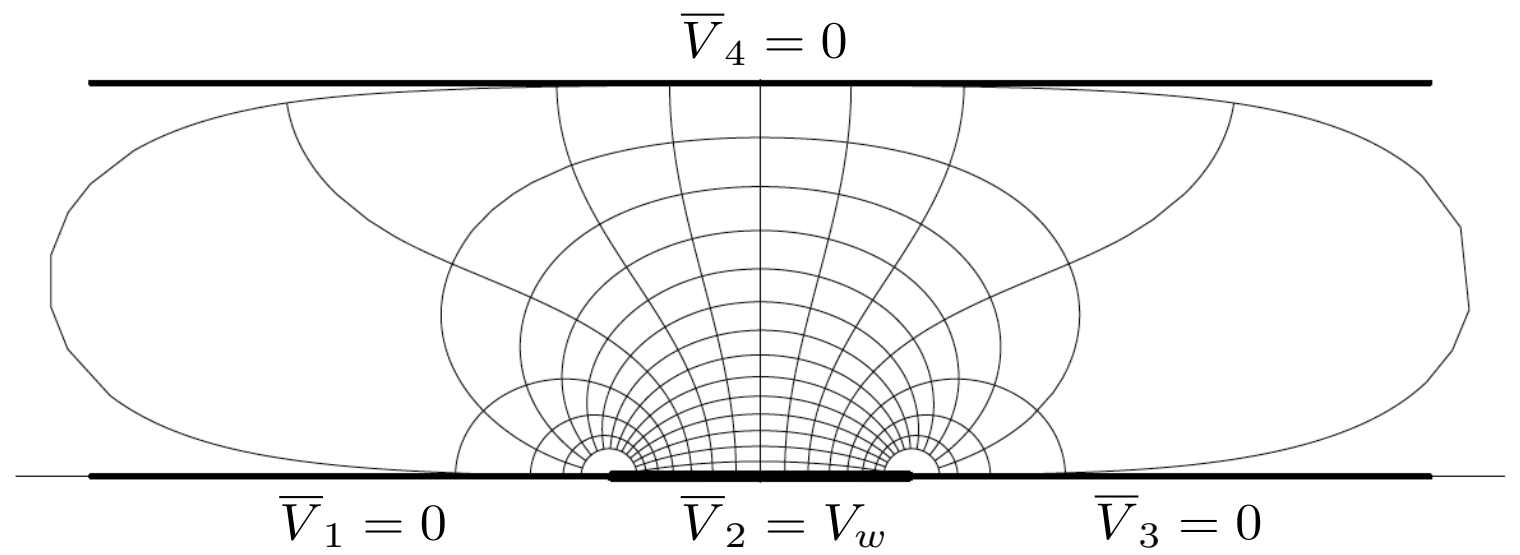

Figure 2.5: Illustration showing the equipotential lines of the weighted field produced when electrode 2 is raised to potential $V_{w}=1 \mathrm{~V}$ with the rest of the conductors grounded [19].

The total induced charge that flows through electrode 2 due to the moving point charge was determined by integrating Equation 2.26. By defining that the time for the point charge to move from $x_{0}$ to $x_{1}$ occurs during the interval $t=\left[t_{0}, t_{1}\right]$ gives the integral as

$$
\begin{gathered}
Q_{2}^{i n d}=\int_{t_{0}}^{t_{1}} I_{2}^{i n d} d t \\
Q_{2}^{i n d}=-\frac{q}{V_{w}} \int_{t_{0}}^{t_{1}} \vec{E}_{2}[x(t)] \cdot \vec{v}(t) d t, \\
Q_{2}^{i n d}=-\frac{q}{V_{w}} \int_{t_{0}}^{t_{1}}\left[-\nabla \psi_{2}(x)\right] \cdot \frac{d \vec{x}}{d t} d t,
\end{gathered}
$$




$$
\begin{gathered}
Q_{2}^{i n d}=\frac{q}{V_{w}} \int_{x_{0}}^{x_{1}}\left[\nabla \psi_{2}(x)\right] \cdot d \vec{x}, \\
Q_{2}^{i n d}=\frac{q}{V_{w}}\left[\psi_{2}\left(x_{1}\right)-\psi_{2}\left(x_{0}\right)\right] .
\end{gathered}
$$

The result in Equation 2.27 shows that the total induced charge that flows through electrode 2 is independent of the path the point charge follows but only dependent on the two end points of the trajectory.

In the previous example only the induced current and charge for electrode 2 is considered, however as shown in the case for the stationary point charge, an alternate system can always be considered so that any conductor $i$ produces the weighted field. Analogous to the case presented in Section 2.2, Equation 2.26 can be generalized to $[20]$

$$
I_{i}^{i n d}=-\frac{q}{V_{w}} \vec{E}_{i}[x(t)] \cdot \vec{v}(t),
$$

where $I_{i}^{\text {ind }}$ is the induced current that flows through conductor $i$ and $\vec{E}_{i}[x(x)]$ is the weighted field produced by conductor $i$ in the system where the charge has been removed. Similarly, the total charge that flows through conductor $i$ due to the motion of the charged particle is generalized as [18]

$$
Q_{i}^{i n d}=\frac{q}{V_{w}}\left[\psi_{i}\left(x_{1}\right)-\psi_{i}\left(x_{0}\right)\right] .
$$

The findings presented in Equations 2.28 and 2.29 are both variations of Ramo's theorem for the case of a moving point charge. The theorem states that the induced current that flows through a grounded conductor due to a passing point charge is proportional to the weighted field produced by that electrode in a system where the charge has been removed. Furthermore, it states that the total charge that flows 
through that conductor is directly proportional to the end points of the point charge's trajectory and independent of the path taken. Ramo's theorem is utilized in the first step of simulating the sTGC's to determine the initial charge induced on all electrodes due to drifting charged clusters within the detector. 


\section{Chapter 3}

\section{Physics of a MWPC}

\subsection{Fundamentals of a MWPC}

A Multiwire Proportional Chamber (MWPC) is a gaseous particle detector used to reconstruct charged particles that pass through its gas volume. The basic structure of a MWPC is shown in Figure 3.1, where anode wires are suspended between two cathode planes with the entire volume being filled with gas. The cathode-anode separation as well as the anode wire spacing are both on the order of millimetres and the diameter of the wires are typically on the micrometre scale. The wires are held at a high voltage producing an electric field in the space between the gap. By setting

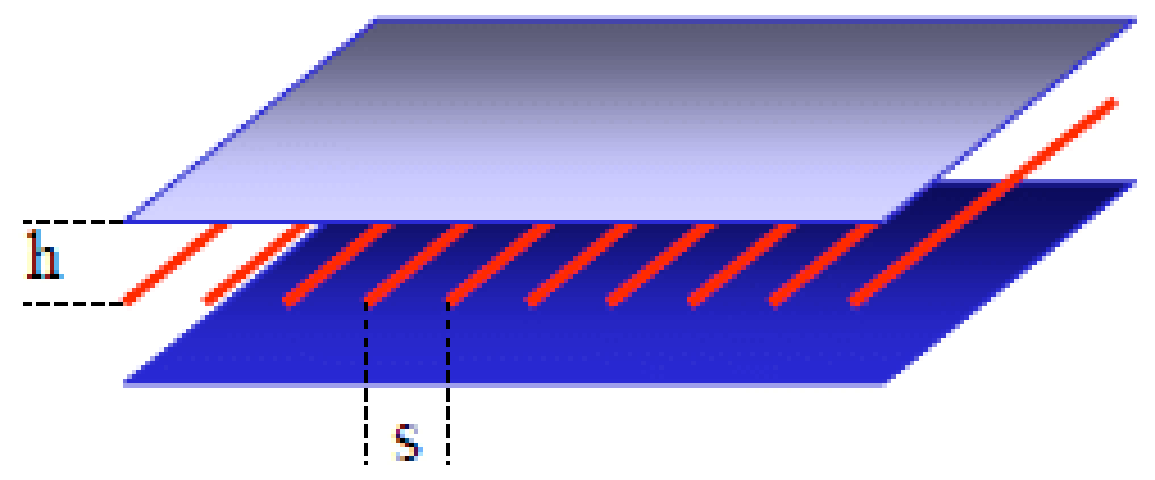

Cathode

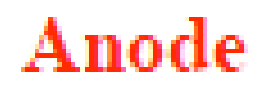

Cathode

Figure 3.1: Basic design of a MWPC. Here anode wires are equally spaced between two cathode planes. The wire to plane spacing is given by $h$ and the centre-tocentre spacing of the wires is denoted by $s[21]$. 
the cathode planes to ground the electric field that is produced by the wires inside the MWPC is illustrated in Figure 3.2. As a charged particle passes through the chamber it will produce ionization clusters within the gas and these newly liberated electrons will begin to drift towards the wires following the field lines illustrated in Figure 3.2, while the ions will drift towards the cathode planes. In the presence of the electric

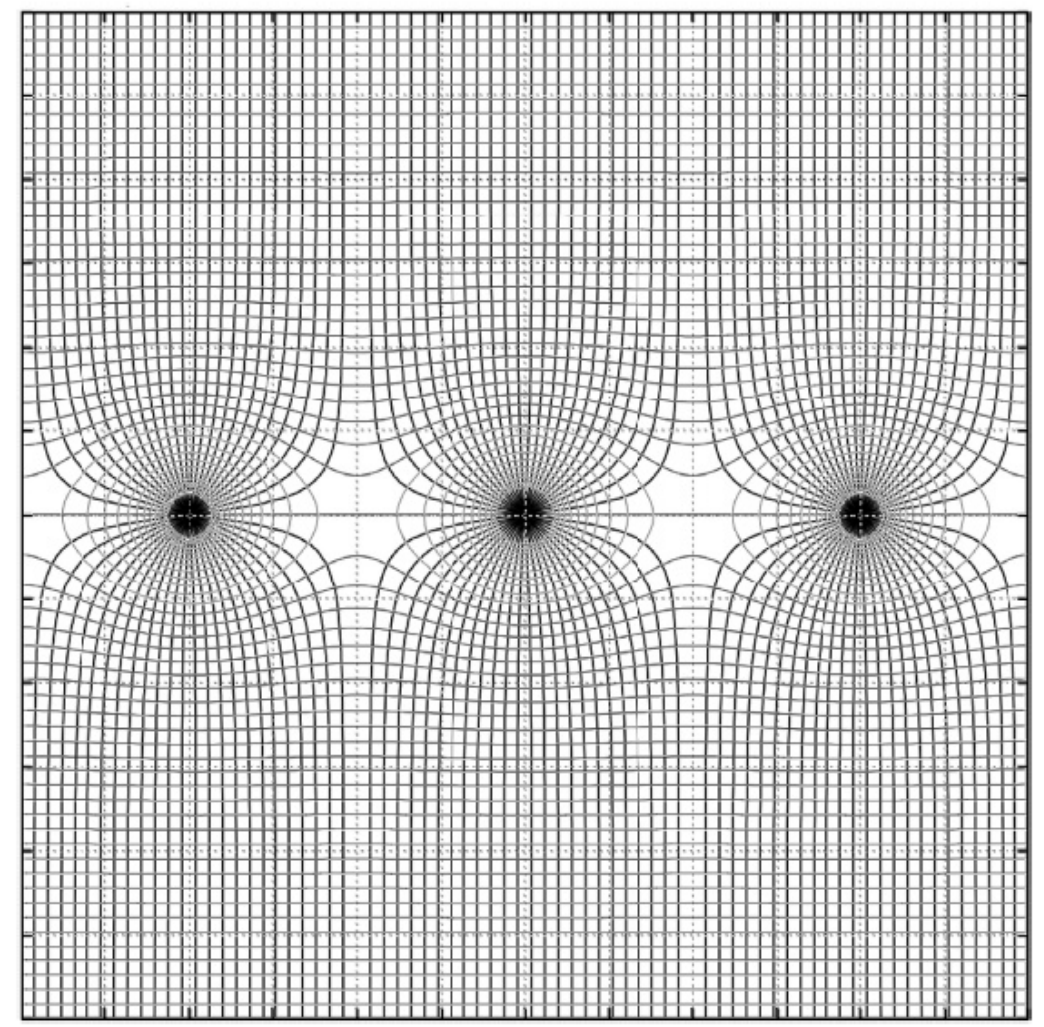

Figure 3.2: Electric field and equipotential lines within a MWPC. Near a wire the field, as expected, is characteristic to the electric field produced by a wire with a $1 / r$ dependence. Moving away from the wire, the field becomes uniform as if the wire plane is continuous and held at a constant potential. A distortion in the field can also be seen when a wire is slightly displaced [22]

field the ionized electrons will begin to gain energy accelerating towards the wire. As they approach within very close proximity of the wire the electrons will have gained enough energy to further ionize the gas producing an electron avalanche at the wire. In accordance with Ramo's theorem, these drifting electron/ion pairs will induce charge on neighbouring electrodes. The wire that collects the drifting electrons will readout 
a negative amplitude signal. The other wires will acquire a positively induced charge on their surface resulting in a positive amplitude signal. This difference in polarity of the signals allows for a reference point of where the avalanche and ionization occur within the detector. Since the electrons have a greater drift velocity than their ion counterparts the signals produced on the cathodes will have a quick spike due to the electrons followed by a long tail due to the ions.

A single anode layer MWPC can only provide a single coordinate with respect to where the ionization location occurs. The second coordinate can be obtained by adding another wire layer perpendicular to the initial layer. Another method, which is implemented in the sTGC, is using segmented cathode strips perpendicular to the wires along one cathode. This technique will be explained more in Chapter 4. To complete full track reconstruction, many MWPC's are stacked together to allow for measurement of several ionization locations along the particles trajectories.

\subsection{Ionization and Transport Properties}

\subsubsection{Ionization}

As outlined above, the principle mechanism for the detection of charged particles will be the measurement of ionization produced in the gas volume. As the charged particle passes through the detector it will interact electromagnetically with the gas molecules to produce ionization clusters within the gas. The number of clusters that will be produced and their size are both statistical in nature. The charged particle will deposit energy via collisions with the gas molecules producing primary ionization electrons. The frequency of primary ionization events through the detection region will follow a Poisson distribution. The primary electrons can further ionize neighbouring gas molecules if the initial energy deposition is large enough. The amount of secondary ion pairs that will be produced describes the size of the cluster 
and will follow a Landau distribution [23].

The amount of energy that will be deposited in the gas volume by a passing charged particle is a result of several factors. The average energy loss per unit length, $d E / d x$, will be given by the empirical Bethe-Bloch equation [23]

$$
\frac{d E}{d x}=\frac{4 \pi n_{e} e^{4}}{m c^{2}}\left(\frac{\mathcal{Z}}{\beta}\right)^{2}\left[\ln \frac{2 m c^{2} \beta^{2} \gamma^{2}}{I}-\beta^{2}-\frac{\delta(\gamma)}{2}\right],
$$

where $n_{e}$ is the electron number density of the gas, $\mathcal{Z}$ is the charge of the incident particle, $c$ is the speed of light, $e$ is the elementary charge constant, and the $m$ is the electron mass. $\beta$ and $\gamma$ are the speed of the incident particle in terms of $c$ and the

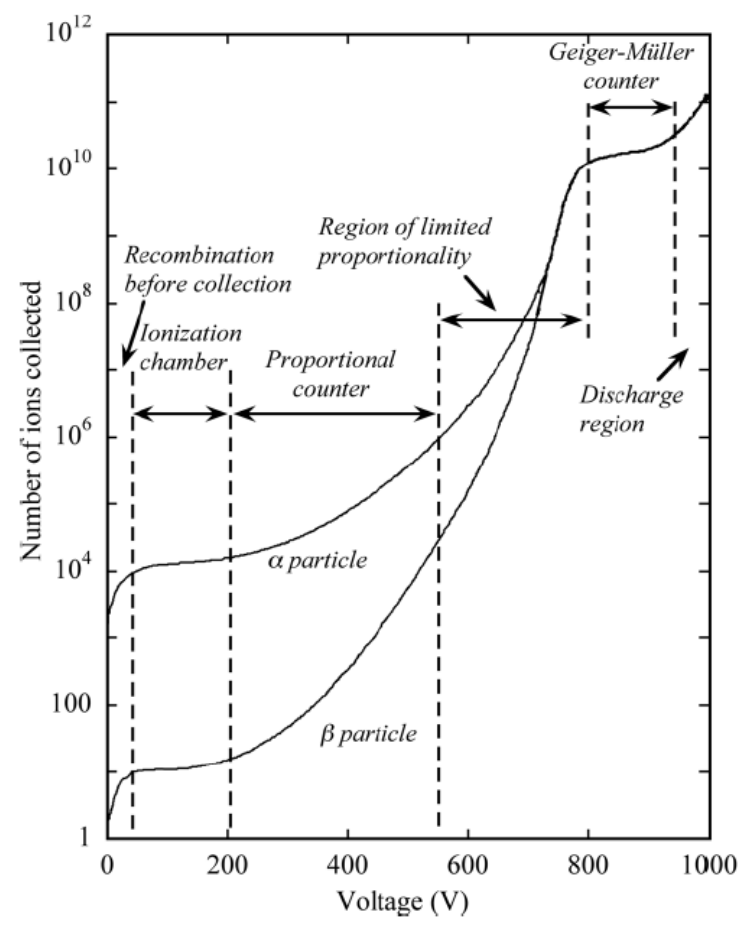

Figure 3.3: Collection of electron/ion pairs at electrodes as a function of voltage in a proportional counter. Highlighted are the voltage regions for specific operational modes [22].

Lorentz factor, respectively. The average energy required to ionize a gas molecule is given by the mean ionization potential $I . \delta(\gamma)$ is a positive correction term dependent on $\gamma$ to account for higher density mediums with small spacing between molecules. 
From Equation 3.1 it is seen that the amount of energy loss will increase with $\gamma$, the correction term will cause the energy loss to plateau for faster moving charged particles with a large $\gamma$ value.

In a MWPC the potential of the wires must be set such that the electron/ion pairs will gain enough energy to avoid recombination allowing a signal to be detected. Illustrated in Figure 3.3, the number of electrons that will be collected at the wire (or ions at the cathode) is a function of the applied voltage. If the voltage is set too low the pairs will not have enough energy to accelerate away from each other and will recombine. In the next region the pairs will drift toward their respective electrodes, however the primary electrons will not have the energy required to produce secondary ionization resulting in a small signal. In the proportional counter region, the ionized electrons will gain enough energy accelerating towards the wire to produce a gain of $10^{5}$ secondary electrons within a few wire radii of the wire [22]. This will result in a much larger signal than the ionization chamber that is proportional to the amount of initial ionization. In the Geiger-Müller region the secondary ionization that will be produced is amplified due to the large electric field however the signal will lose all proportionality to the energy deposited by the charged particle that traversed the MWPC. Once the voltage is high enough, the electric field inside the chamber will be so great that ionization will occur independently from outside influence [23]. The key operating conditions of a MWPC will be in the proportional counter region to allow for amplified ionization detection for track reconstruction.

\subsubsection{Drift}

Once the gas molecules are ionized the electron/ion pairs will begin to drift and diffuse in the gas volume. In the absence of an electric field both the electrons and ions will only have a thermal component to their velocity. From the Maxwell-Boltzmann 
distribution the average thermal velocity of the electron/ion is given by [22]

$$
\bar{v}_{\text {thermal }}=\sqrt{\frac{8 k T}{\pi m}}
$$

where $k$ is the Boltzmann constant, $T$ is the temperature, and $m$ is the mass. As can be inferred from Equation 3.2, the electron will have a greater thermal velocity than the ion. Furthermore, the ionization cluster will diffuse longitudinally following a Gaussian distribution with a standard deviation along the drift direction of [22]

$$
\sigma_{x}=\sqrt{2 D t}
$$

In Equation 3.3, $t$ is the drift time and $D$ is the diffusion coefficient. The diffusion coefficient is an intrinsic property that will vary with different gases or gas mixtures.

In the presence of an electric field the electron and ions will accelerate along the field lines to their respective electrodes. The average drift speed the ions will attain is given by $[22]$

$$
v_{d r i f t}^{\text {ion }}=\mu E,
$$

where $E$ is the electric field strength and $\mu$ is the ion mobility. The mobility will depend on the specific ion moving through a given gas and is proportional to the diffusion coefficient. Classically, the mobility is related to the diffusion coefficient by $[24]$

$$
\mu=\frac{D e}{k T}
$$

The distribution of the drifting ions will be Gaussian with a standard deviation of [22]

$$
\sigma_{x}=\sqrt{\frac{2 k T}{e} \frac{x}{E}}
$$


where $x$ is the distance the ion cluster will travel in a given time interval and the rest are as above. Due to their size, the drift velocity of the electrons will be much greater than the ions since they attain more energy between collisions. Electrons will achieve a drift velocity of $[22]$

$$
v_{\text {drift }}^{\text {electron }}=k \frac{e E}{m} \tau .
$$

In Equation $3.7 m$ is the electron mass, $k$ is a constant between between 0.75 and 1 that depends on assumptions of the energy distribution of the electrons [22], and $\tau$ is the mean time between collisions. Electron clusters will be Gaussian and linearly diffuse following Equation 3.6. These descriptions of the transport properties of ionization in a gaseous detector are based on classical assumptions, in reality there is no analytical solution to describe the drift velocity or longitudinal and transverse diffusion properties of ionization clusters in a given pure gas or gas mixture. The behaviour of the ionization clusters is based purely on empirical evidence from experiments to study these properties for various gases. The effects of the electric field on electron drift velocity and longitudinal diffusion are illustrated in Figure 3.4.

The choice of fill gas is important in the design of a MWPC depending on its purpose. To reconstruct one coordinate of the initial ionization location the arrival time of the electrons to the wires is used. However, as the electron approaches, it moves through regions of increasing electric field. Therefore the behaviour of the electron cluster in the gas needs to be understood for accurate timing measurements. For example, in Figure 3.4a, in a gas mixture of 90:10 $\mathrm{Ar}: \mathrm{CO}_{2}$ the drift velocity rapidly increases in the region of low electric field and then remains relatively constant up to several $\mathrm{kV} / \mathrm{cm}$. Conversely, the drift velocity in a mixture of 10:90 Ar: $\mathrm{CO}_{2}$ continually increases resulting in the electron constantly accelerating towards the wire. Furthermore, as depicted in Figure 3.4b, the longitudinal diffusion of the electron cluster depends on the electric field. A method, to be explained in Chapter 4, used to 


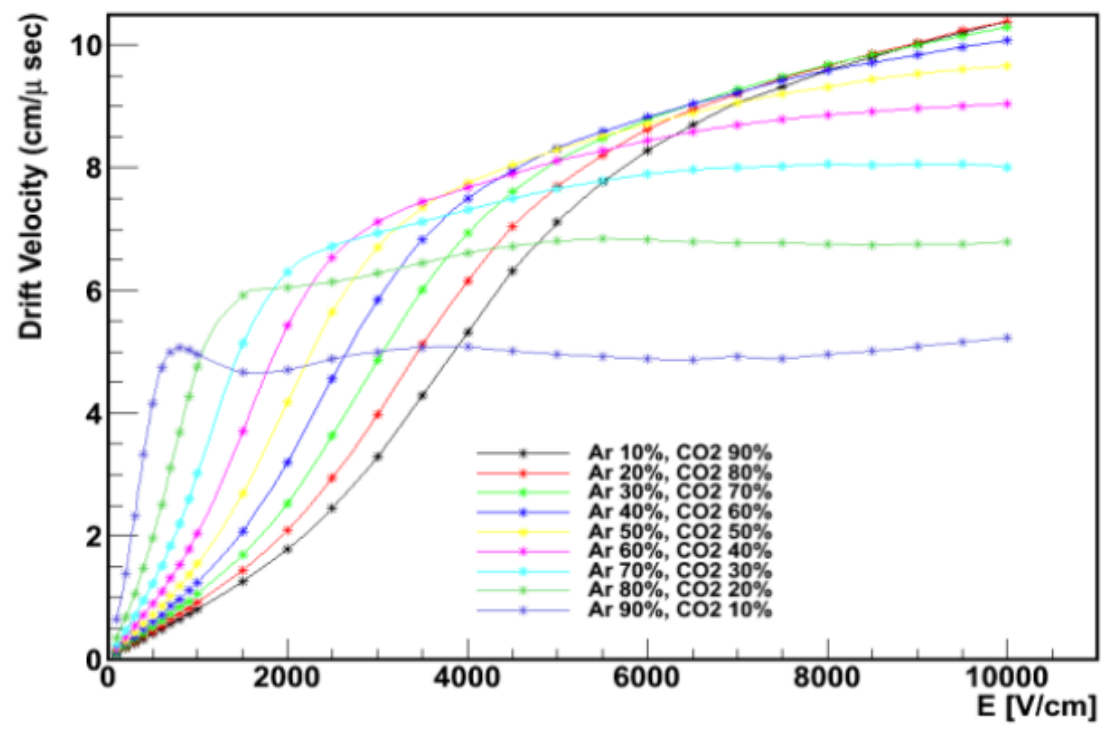

(a)

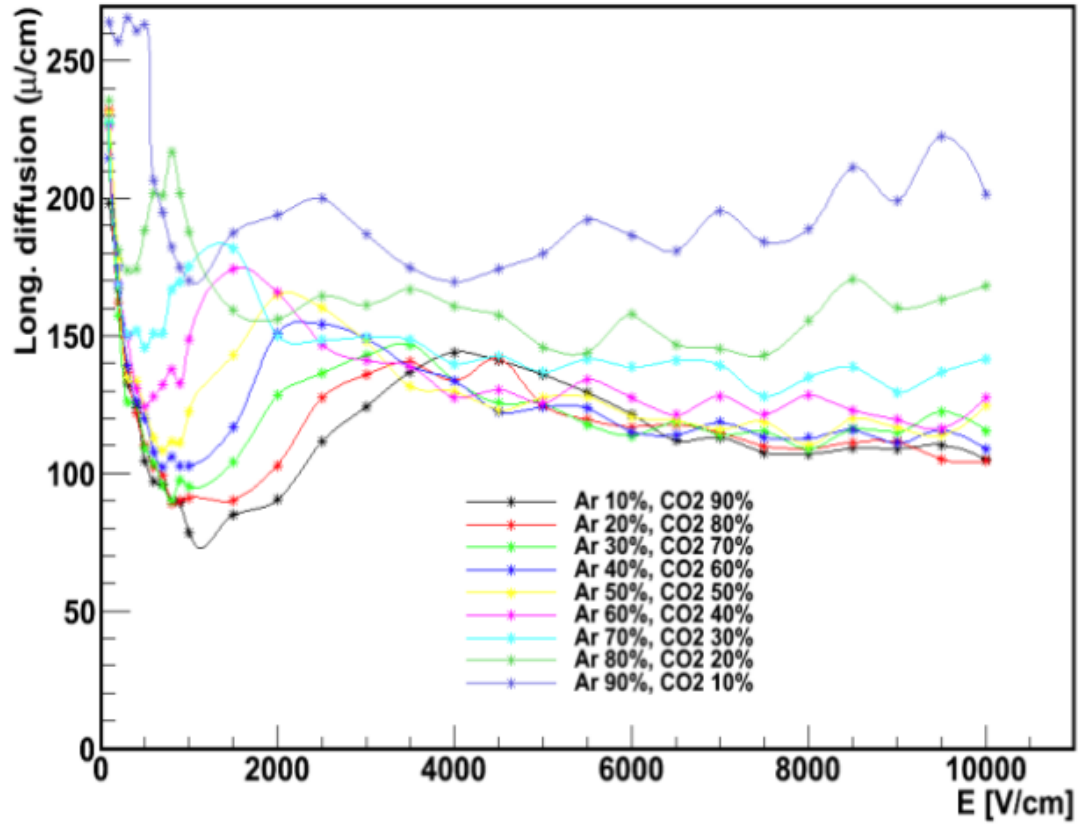

(b)

Figure 3.4: (a) Electron drift velocity in mixtures of Argon and $\mathrm{CO}_{2}$ as a function of electric field. (b) Electron longitudinal diffusion in mixtures of Argon and $\mathrm{CO}_{2}$ as a function of electric field [25]. 
determine the second coordinate involves the induction of charge on segmented cathode strips. If the electron cluster disperses significantly it will greatly reduce the measurement resolution of this second coordinate. Referring to Figure 3.4b the mixture of 90:10 Ar: $\mathrm{CO}_{2}$ maintains relatively large diffusion compared to the mixture of 10:90 Ar: $\mathrm{CO}_{2}$. Balancing these two effects is important in the design of a MWPC.

\subsubsection{Wire Amplification}

While the electrons drift toward the wire they begin to accelerate and gain energy. Once electrons exceed the energy of the ionization potential of the gas they begin to produce secondary ionization. This process occurs within a few wire radii of the wire and is referred to as an electron avalanche. In Figure 3.5, the progress of the avalanche
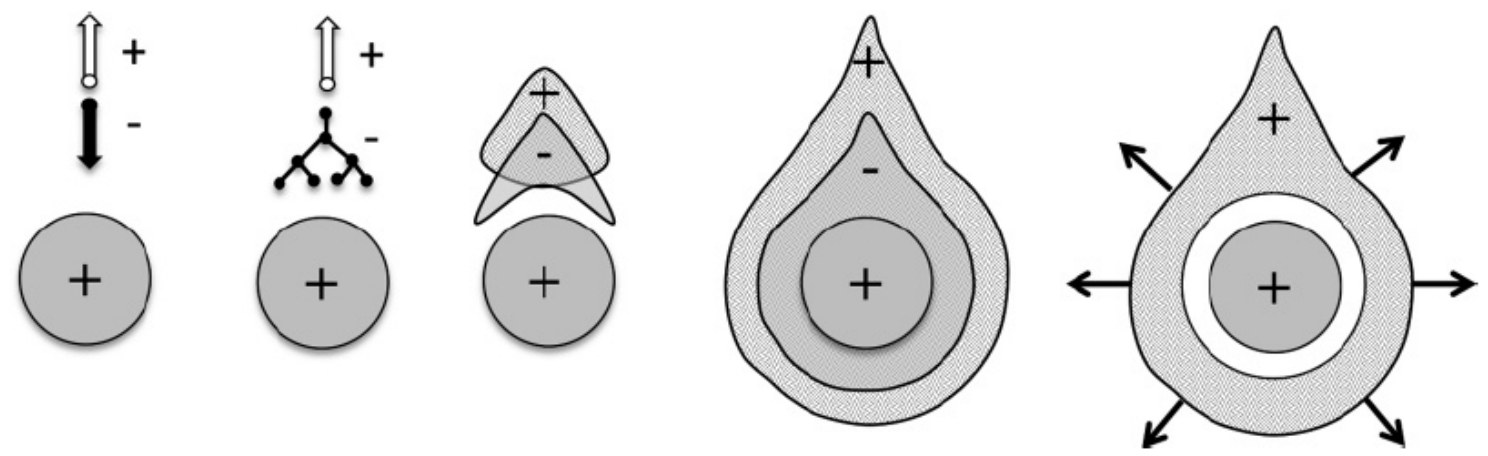

Figure 3.5: Once primary ionization occurs the electron drifts towards the wire gaining energy. Secondary ionization occurs within close proximity to the wire producing an electron avalanche. Due to the small radius of the wire the ionized electrons typically engulf the wire [22].

from initial ion production is depicted. Since the electrons have much larger drift velocities than their ion counterparts the avalanche cloud forms into a "liquid drop" around the wire. The electrons are then collected by the wire and the ions drift towards the cathode.

The probability that an electron produces ionization over a distance $x$ is given by the first Townsend coefficient $\alpha$. This coefficient is the inverse of the mean free path 
between ionizing collisions, $\lambda$, and is proportional to the electric field in a MWPC for a given gas. As a cluster of $n^{\prime}$ electrons drift a distance $d x^{\prime}$ in a uniform field the number of new electrons produced is [24]

$$
d n^{\prime}=n^{\prime} \alpha d x^{\prime} .
$$

Rearranging Equation 3.8 and integrating both sides

$$
\begin{gathered}
\int_{n_{0}}^{n} \frac{d n^{\prime}}{n^{\prime}}=\int_{x^{\prime}=0}^{x^{\prime}=x} \alpha d x^{\prime} \\
\ln \frac{n}{n_{0}}=\alpha x \\
\frac{n}{n_{0}}=e^{\alpha x},
\end{gathered}
$$

where $n$ is the total number of secondary electrons produced after $n_{0}$ initial electrons travel a distance $x$. The ratio $n / n_{0}$ in Equation 3.9 is the multiplication factor, $M$, also referred to as the gas gain and is evidently equivalent to $e^{\alpha x}$. In the situation more akin to a MWPC, the Townsend coefficient is a function of $x$ in a non uniform field. The gas gain then becomes [24]

$$
M=\exp \left[\int_{r_{1}}^{r_{2}} \alpha(x) d x\right],
$$

where $r_{1}$ and $r_{2}$ are the initial and final positions of the initial electron drift, respectively. Shown in Figure 3.6 are the first Townsend coefficients at different electric field values in specific gases and mixtures. Along with the drift velocity and diffusion of the electron cluster, the required amplification of the fill gas must also be considered in the design of the MWPC. In Equation 3.9 the gas gain appears to increase without limit, however as the abundance of charge increases within the gap, a deformation 
in the electric field occurs resulting in spark breakdown within the detector. The multiplicative limit is set by the Raether condition of $M \leq 10^{8}$ before breakdown occurs with an operation limit of $M \leq 10^{6}[26]$.

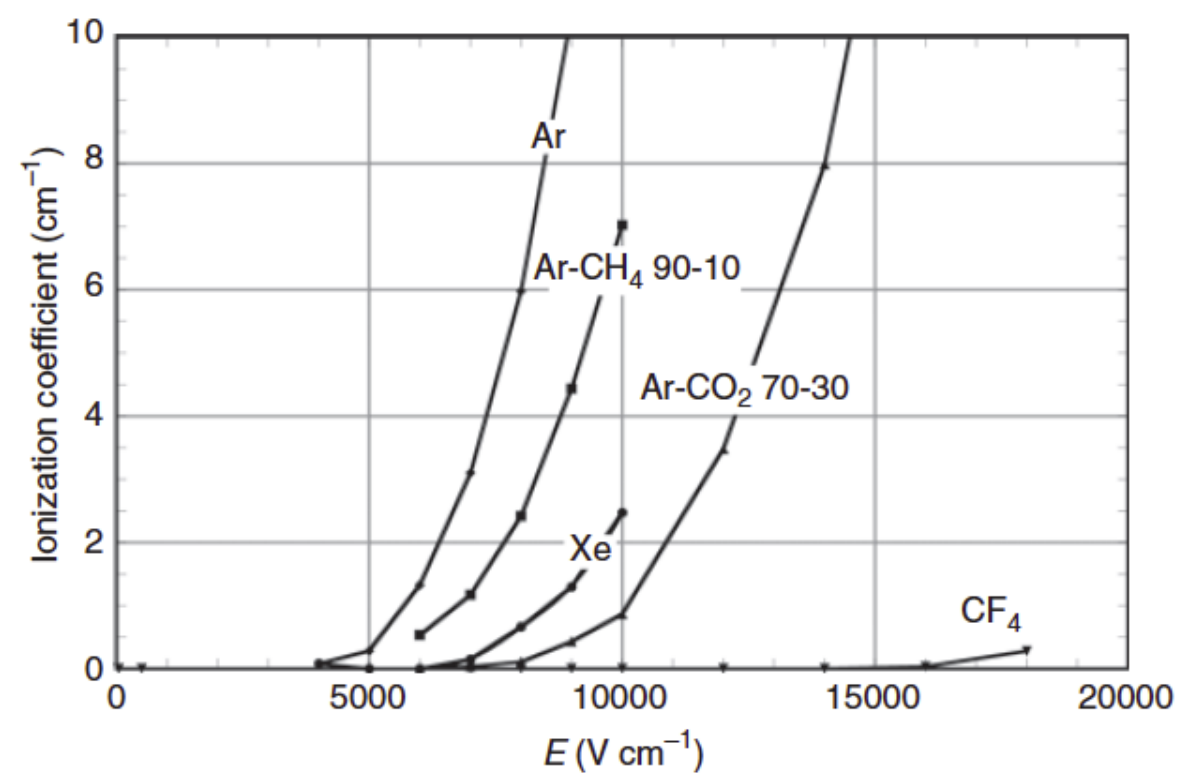

Figure 3.6: Townsend coefficients of various gases and gas mixtures as a function of electric field [22].

\subsection{Charge Dispersion on Resistive Layer}

\subsubsection{Symmetric Ground}

The rapid production of electrons on the wire due to the avalanche will induct charge on the surface of the cathode in accordance with Ramo's theorem. In specific applications, the cathode planes are covered in a resistive layer to protect the electronics from discharge in the high electric field environment. A model was developed by the ILC group at Carleton University to explain how charge disperses on the resistive layer of the Micro Pattern Gas Detector (MPGD) to be used in a TPC for the International Linear Detector (ILD) at the ILC [27][28]. This technique is analogous to MWPC's as both detector technologies implement signal readout electronics of 
the cathodes that measure the charge inducted on the resistive surface. The readout signal is used to position where the ionization occurred within the detector. Upon induction the charge is not fixed to its initial location but flows over the cathode and will diffuse following the two dimensional diffusion equation

$$
\frac{\partial \rho}{\partial t}=\frac{1}{R C}\left(\frac{\partial^{2} \rho}{\partial x^{2}}+\frac{\partial^{2} \rho}{\partial y^{2}}\right),
$$

where $\rho$ is the charge density as a function of position and time, $R$ is the resistance of the layer, and $C$ is the capacitance per unit area between the layer and the cathode. The resistive layer sits on a glue or other protective layer that covers the cathode board between the two surfaces and this gap creates a capacitive coupling between the layers. The $R C$ time constant of this coupling controls how the charge disperses over the cathode.

In a system where the cathode is symmetrically grounded on all sides the induced charge will disperse isotropically over the resistive layer, as illustrated in Figure 3.7.

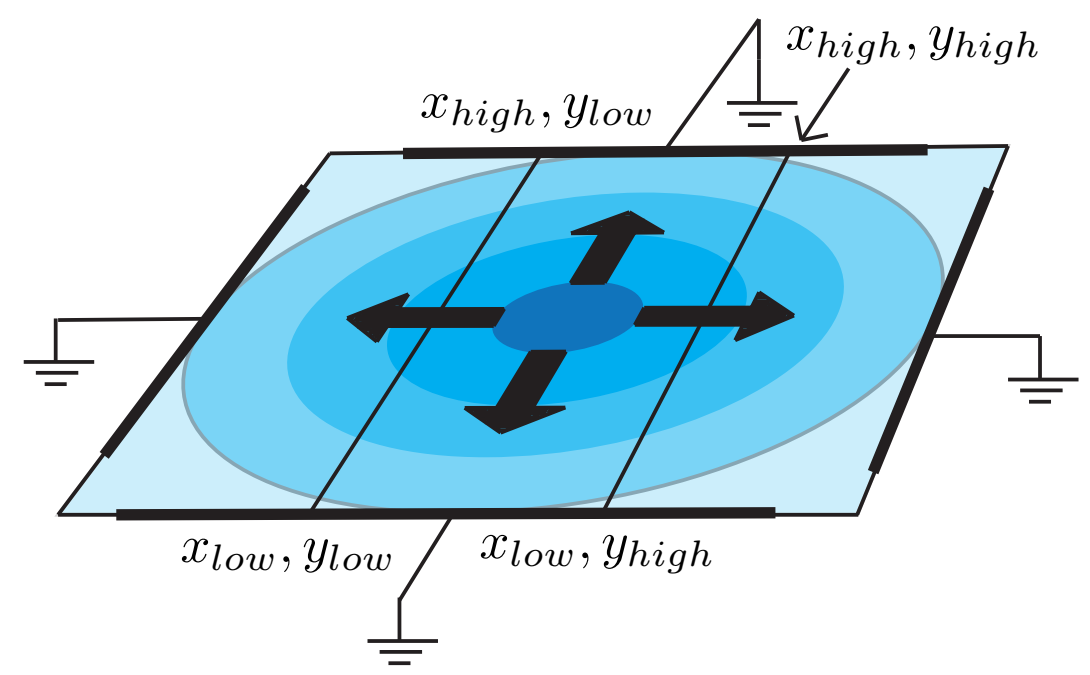

Figure 3.7: Isotropic charge dispersion over a resistive layer with symmetric ground. The darker blue specifies the region with higher charge density. The charge $Q(t)$ on a given cathode strip can be determined by integrating the charge density on the surface over the boundaries of the specific strip. 
Assuming charge is induced as a delta function spike at the coordinates $x=y=0$ at $t=0$ and the cathode edges are at infinity, a solution to Equation 3.11 is given by $[29]$

$$
\rho_{\delta}(x, y, t)=\left(\frac{1}{4 \pi h t}\right) \exp \left[-\frac{\left(x^{2}+y^{2}\right)}{4 h t}\right] .
$$

In Equation 3.12, $h$ is the inverse time constant, $1 / R C$, of the coupling. In reality the induced charge is not localized to an infinitesimal area but follows a Gaussian distribution over the surface with a width proportional to the diffusion of the cluster. A better model of the charge density dispersion is obtained by convoluting Equation 3.12 with a two dimensional Gaussian with width $w$, that is

$$
\rho(x, y, t)=\rho_{\delta}(x, y, t) \otimes G(x, y),
$$

where $G(x, y)$ is given by

$$
G(x, y)=\left(\frac{1}{2 \pi w^{2}}\right) \exp \left[-\frac{\left(x^{2}+y^{2}\right)}{2 w^{2}}\right] .
$$

Solving Equation 3.13 for a charge cluster with charge $N e$,

$$
\begin{gathered}
\rho(x, y, t)=\int_{-\infty}^{\infty} \int_{-\infty}^{\infty}\left(\frac{N e}{4 \pi h t}\right) \exp \left[-\frac{\left[\left(x-x^{\prime}\right)^{2}+\left(y-y^{\prime}\right)^{2}\right]}{4 h t}\right]\left(\frac{1}{2 \pi w^{2}}\right) \exp \left[-\frac{\left(x^{\prime 2}+y^{\prime 2}\right)}{2 w^{2}}\right] d x^{\prime} d y^{\prime} \\
\rho(x, y, t)=\frac{N e}{2 \pi\left(2 h t+w^{2}\right)} \exp \left[-\frac{\left(x^{2}+y^{2}\right)}{2\left(2 h t+w^{2}\right)}\right]
\end{gathered}
$$

where $N$ is the number of electrons in the induction cluster and $e$ is the electron charge. Equation 3.15 satisfies Equation 3.11 with 


$$
\begin{gathered}
\frac{\partial \rho}{\partial t}=\frac{N e h}{2 \pi\left(2 h t+w^{2}\right)^{3}} \exp \left[-\frac{\left(x^{2}+y^{2}\right)}{2\left(2 h t+w^{2}\right)}\right]\left(x^{2}+y^{2}-2\left(2 h t+w^{2}\right)\right) \\
\frac{\partial^{2} \rho}{\partial x^{2}}=\frac{N e}{2 \pi\left(2 h t+w^{2}\right)^{3}} \exp \left[-\frac{\left(x^{2}+y^{2}\right)}{2\left(2 h t+w^{2}\right)}\right]\left(x^{2}-\left(2 h t+w^{2}\right)\right) \\
\frac{\partial^{2} \rho}{\partial y^{2}}=\frac{N e}{2 \pi\left(2 h t+w^{2}\right)^{3}} \exp \left[-\frac{\left(x^{2}+y^{2}\right)}{2\left(2 h t+w^{2}\right)}\right]\left(y^{2}-\left(2 h t+w^{2}\right)\right)
\end{gathered}
$$

If the cathode is segmented into strips, Equation 3.15 can be integrated over the area to give the total charge on a strip as a function of time as [29]

$$
\begin{aligned}
Q(t)= & \frac{N e}{4}\left[\operatorname{erf}\left(\frac{x_{\text {high }}}{\sqrt{2\left(2 h t+w^{2}\right)}}\right)-\operatorname{erf}\left(\frac{x_{\text {low }}}{\sqrt{2\left(2 h t+w^{2}\right)}}\right)\right] \times \\
& {\left[\operatorname{erf}\left(\frac{y_{\text {high }}}{\sqrt{2\left(2 h t+w^{2}\right)}}\right)-\operatorname{erf}\left(\frac{y_{\text {low }}}{\sqrt{2\left(2 h t+w^{2}\right)}}\right)\right], }
\end{aligned}
$$

where erf is the error function and $x_{\text {high }}, x_{\text {low }}, y_{\text {high }}$, and $y_{\text {low }}$ define the boundaries of the strip.

This model was used to accurately simulate the charge dispersion on the resistive layer in a MPGD prototype for the ILD TPC [27][28]. Highlighted in Figure 3.8 is the comparison of a simulated signal to the signal produced due to an x-ray photon in the MPGD of the Carleton TPC prototype for the ILD [29]. By considering how the charge is induced on the resistive surface and then disperses, the signal is able to be accurately reproduced. 


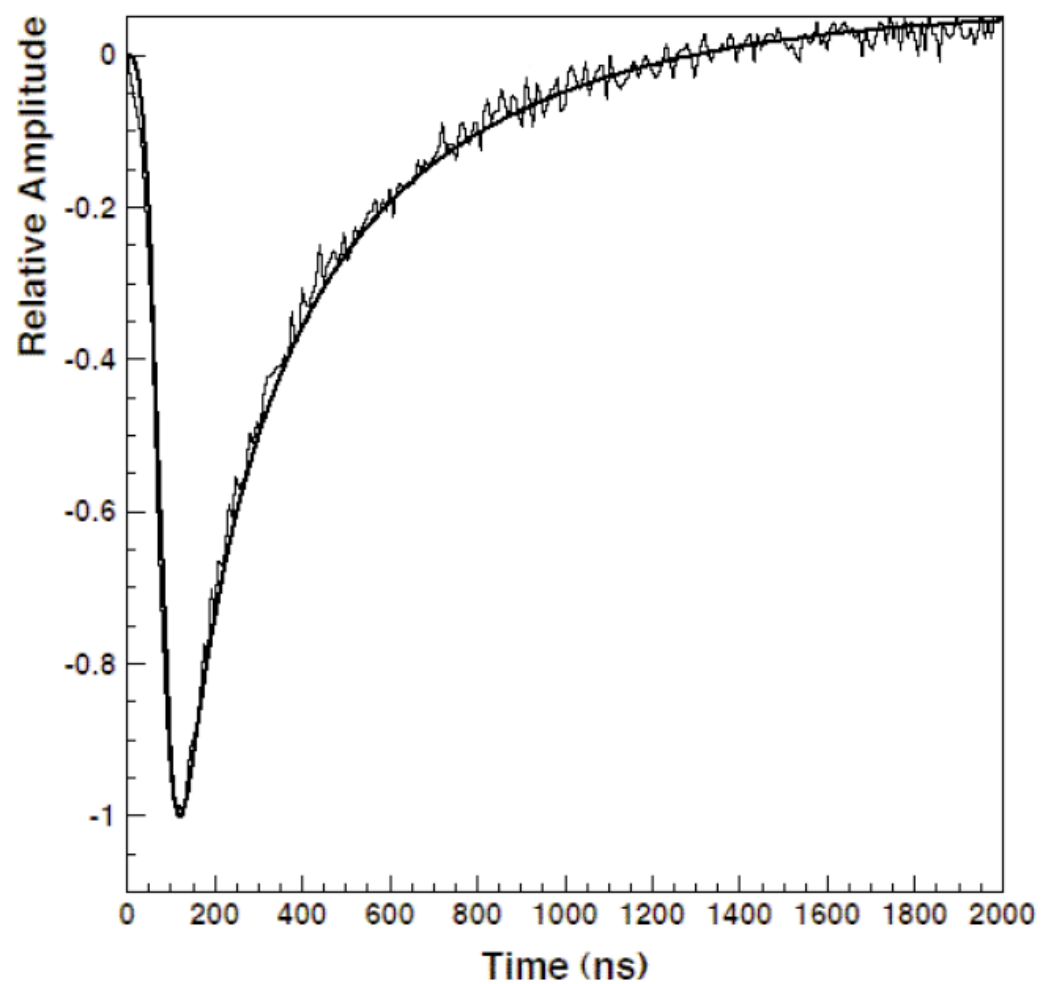

Figure 3.8: Signal simulated using the isotropic charge dispersion model in a MPGD. The thicker line represents the simulated signal and the thin line experimental data [29].

\subsubsection{Ground on One Side}

In the case where a cathode is grounded on one side, the charge dispersion on its surface can be modelled in the following way. When charge is induced on the surface the electric field on it can be determined using the method of images. Assuming in the plane of the induced charge it sees the ground as an infinite sheet perpendicular to its surface. So as not to confuse, the original induced charge will be referred to as "the charge" over the upcoming explanation. Referring to the system shown in Figure $3.9 \mathrm{a}$, the potential due to the charge in the region in front of the sheet is determined by replacing the sheet with a charge $-q$ that is equal distance away from the origin in the opposite direction as illustrated in Figure 3.9b. This gives the potential in the 
area in the front of the sheet as the sum of the two potentials produced by the point charges

$$
V(x, y, z)=\frac{1}{4 \pi \epsilon_{0}}\left[\frac{q}{\sqrt{x^{2}+y^{2}+(z-d)^{2}}}-\frac{q}{\sqrt{x^{2}+y^{2}+(z+d)^{2}}}\right] .
$$

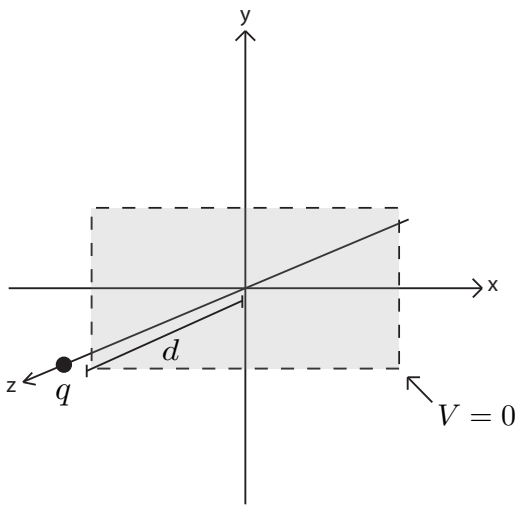

(a)

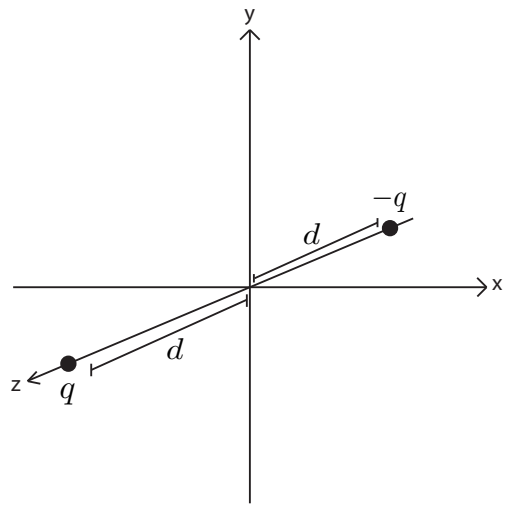

(b)

Figure 3.9: (a) A point charge $q$ a distance $z=d$ in front of an infinite grounded sheet in the $x y$-plane. (b) Infinite sheet replaced with an image charge $-q$ a distance $d$ along the negative $z$ axis.

In Equation $3.20 d$ is the distance from the grounded sheet shown in Figure 3.9a. The electric field on the surface produced by this potential is given by the gradient of Equation 3.20, that is at $z=0$

$$
\begin{gathered}
\vec{E}=-\left.\vec{\nabla} V\right|_{z=0} \\
E_{z}=-\frac{q d}{2 \pi \epsilon_{0}\left(x^{2}+y^{2}+d^{2}\right)^{3 / 2}} .
\end{gathered}
$$

The electric field in Equation 3.21 will only have a $z$-component since that is the only direction normal to the surface. The field lines in this system are shown in 
Figure 3.10. From Equation 3.21 the surface charge distribution is determined using the fact that $\sigma=\epsilon_{0} E$ for an infinite plane results in

$$
\sigma(x, y)=-\frac{q d}{2 \pi\left(x^{2}+y^{2}+d^{2}\right)^{3 / 2}} .
$$

When charge is induced on the cathode it diffuses following Equation 3.22. Since the cathode is covered with a resistive layer its diffusion will be proportional to $h$ defined previously. The charge distribution depends on the distance from ground and this distance is determined by $d=\sqrt{2 h t}$, where $h$ is the inverse time constant and $t$ is the time. This results in the final charge density as a function of time given by

$$
\sigma(x, y, t)=\frac{N e \sqrt{2 h t}}{2 \pi\left(x^{2}+y^{2}+2 h t\right)^{3 / 2}},
$$

where $-q$ was replaced with $N e, N$ being the total number of electrons and $e$ being the electron charge.

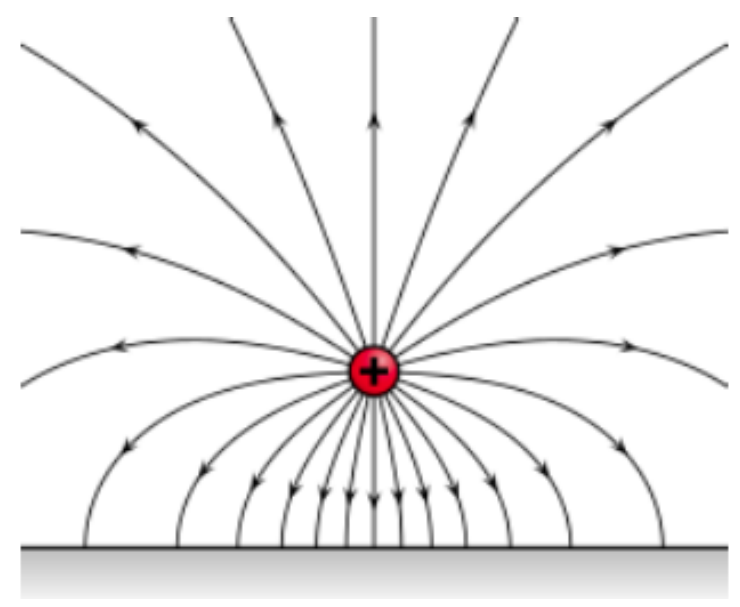

Figure 3.10: Electric field lines when a point charge is in the vicinity of an infinite grounded electrode

For a cathode segmented into strips Equation 3.23 can be integrated to determine the total charge on an individual strip as a function of time. In this case, the strips are taken to be infinite in $x$ and finite in $y$ with their dimensions in $y$ given as $y_{h i g h}$ 
and $y_{\text {low }}$ as illustrated in Figure 3.11 [18]

$$
\begin{gathered}
Q(t)=\int_{y_{\text {low }}}^{y_{\text {high }}} \int_{-\infty}^{\infty} \sigma(x, y, t) d x d y \\
Q(t)=\int_{y_{\text {low }}}^{y_{\text {high }}} \int_{-\infty}^{\infty} \frac{N e \sqrt{2 h t}}{2 \pi\left(x^{2}+y^{2}+2 h t\right)^{3 / 2}} d x d y, \\
Q(t)=\int_{y_{\text {low }}}^{y_{\text {high }}} \frac{N e \sqrt{2 h t}}{\pi\left(y^{2}+2 h t\right)} d y, \\
Q(t)=\frac{N e}{\pi}\left[\tan ^{-1}\left(\frac{y_{\text {high }}}{2 \sqrt{h t}}\right)-\tan ^{-1}\left(\frac{y_{\text {low }}}{2 \sqrt{h t}}\right)\right] .
\end{gathered}
$$

Since the cathodes of the sTGC are segmented into strips and grounded on one side, this model of charge dispersion is used to account for how charge disperses in the simulations.

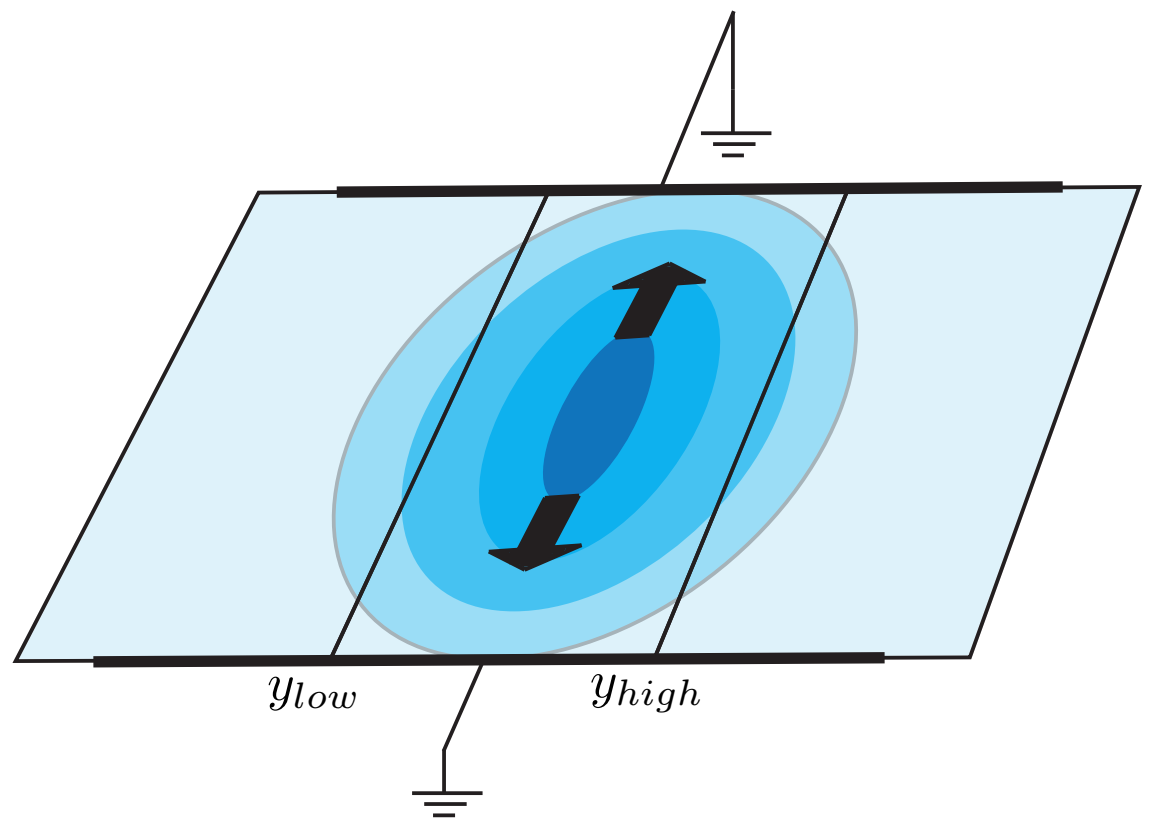

Figure 3.11: Charge dispersion over strips grounded on one side. The darker blue specifies the region with higher charge density. The charge $Q(t)$ on a given cathode strip can be determined by integrating the charge density on the surface over its width and taking its length to infinity. 


\section{Chapter 4}

\section{Small-Strip Thin Gap Chamber}

\subsection{Overview of the sTGC}

The sTGC is a MWPC detector designed for reconstruction in the ATLAS inner end-cap region for muons originating from the IP. As discussed in Section 1.3, the

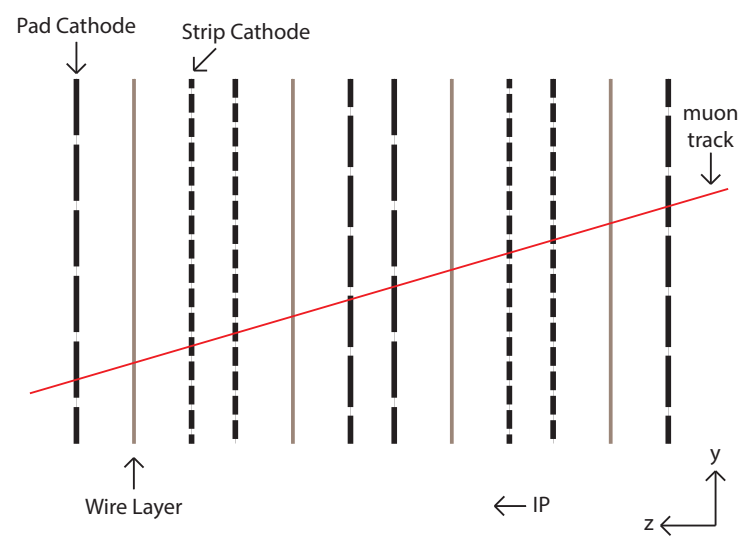

(a)

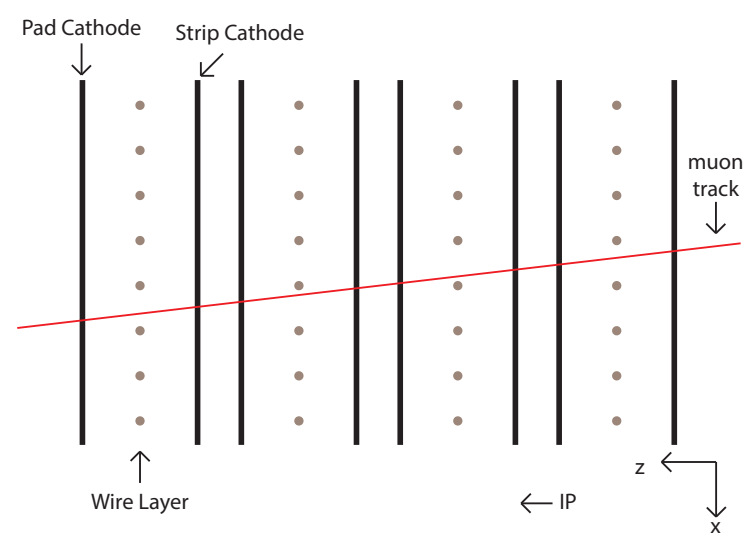

(b)

Figure 4.1: (a) Side view through an sTGC quadruplet. Shown is a muon passing through the four layers, from left to right. In reality cathodes are continuous with pad/strip segmentation occurring in the cathode board, segmentation in picture is for illustrative purpose only and is not to scale. (b) Top-down view of sTGC quadruplet with muon originating from IP. Shown are the individual wires that make up the wire layer, not to scale. 
sTGC quadruplet is an assembly of four individual MWPC gaps and Figure 4.1 highlights the sequence in which a muon will pass through the four layers from left to right. Illustrated in Figure 4.2 is the cross-section of a single sTGC gap. The $50 \mu \mathrm{m}$ gold plated tungsten anode wires have a pitch spacing of $1.8 \mathrm{~mm}$ with an anodecathode separation distance of $1.4 \mathrm{~mm}$ and are held at $2.9 \mathrm{kV}$. The volume between the cathodes is filled with a mixture of 45:55 n-pentane: $\mathrm{CO}_{2}$. Along one cathode $2.7 \mathrm{~mm}$ wide copper strips run perpendicular to the wires with a centre-to-centre spacing of $3.2 \mathrm{~mm}$ while large copper pads, approximately $2-3 \mathrm{~cm}$ wide depending on the quadruplet type, cover the opposite cathode. The design parameters of the sTGC are summarized in Table 4.1.

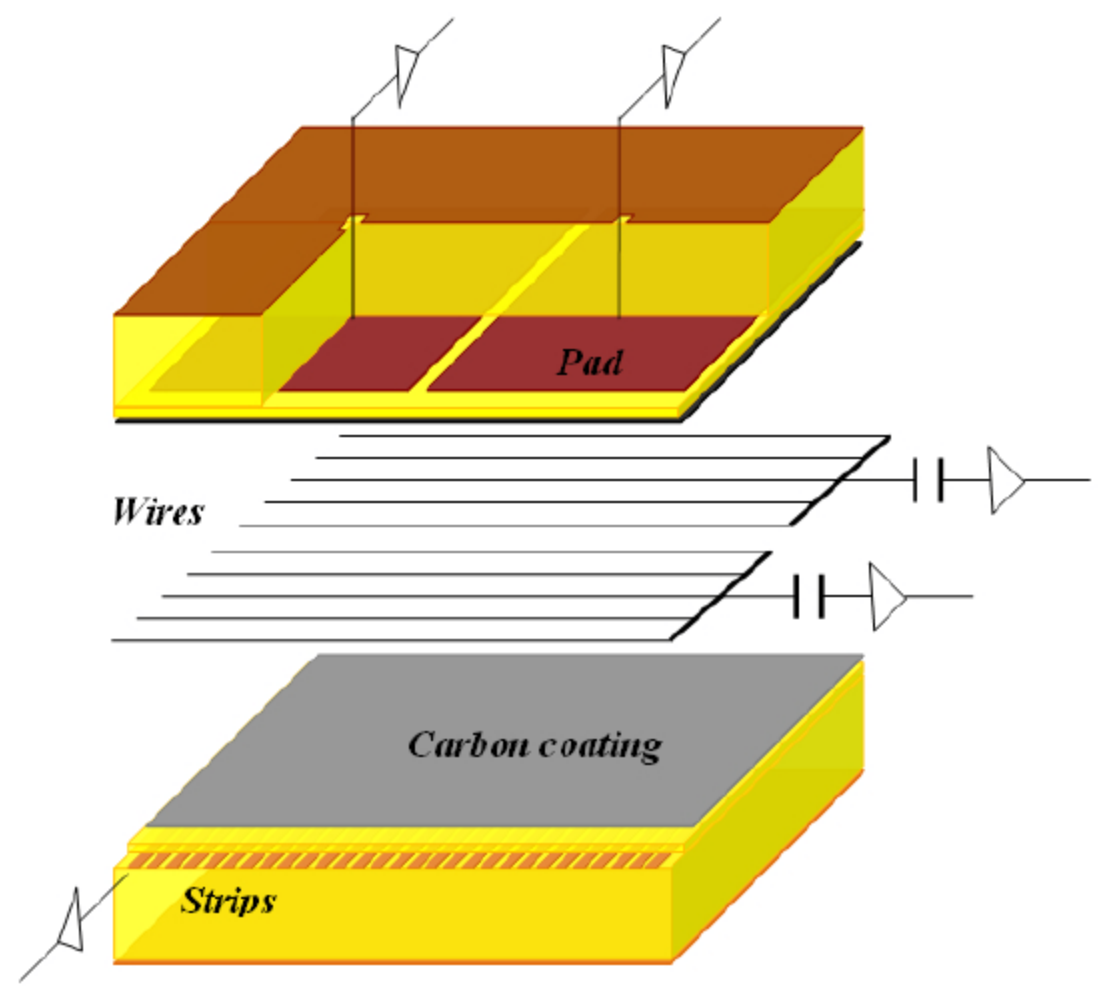

Figure 4.2: Basic schematic cross-section of the sTGC detector. Shown are the strips and pads along the two cathodes and the wires suspended between them. Each cathode is covered with a resistive carbon layer and the volume is filled with a mixture of 45:55 n-pentane: $\mathrm{CO}_{2}$. Figure is not to scale, the defining characteristic of the sTGC is that the wire spacing is on the same scale as the anode-cathode separation distance. The spacing is $1.8 \mathrm{~mm}$ and the separation is $1.4 \mathrm{~mm}$ [30]. 


\begin{tabular}{|c|c|}
\hline Thickness of gas gap & $2.8 \mathrm{~mm}$ \\
\hline Wire pitch & $1.8 \mathrm{~mm}$ \\
\hline Wire diameter & $50 \mu \mathrm{m}$ \\
\hline Wire/cathode distance & $1.4 \mathrm{~mm}$ \\
\hline Copper strip width & $2.7 \mathrm{~mm}$ \\
\hline Strip pitch & $3.2 \mathrm{~mm}$ \\
\hline Wire operating voltage & $2.9 \mathrm{kV}$ \\
\hline
\end{tabular}

Table 4.1: Summary of the design parameters for the sTGC.

The strips and pads serve as readout electrodes for use in reconstruction and triggering, respectively. To ensure muons originate from the IP, a 3-out-of-4 coincidence is required for pad hits in a single quadruplet. The pads serve as the Level-1 trigger in the NSW, when they achieve the 3-out-of-4 hit coincidence they enable groups of strips and wires for readout [14]. The staggered pads overlap in $\phi$ from layer to layer to reduce any discrepancies of a track that passes between two pads in a single layer. The design specifications require a single sTGC layer to have a spatial resolution of $100 \mu \mathrm{m}$ for offline track reconstruction and an angular resolution of $1 \mathrm{mrad}$ for online triggering [31]. The copper strips provide a measurement of the $y(\eta)$ coordinate, the pads provide the $x(\phi)$ coordinate, and the wire that collects the charge resulting from the gas ionization provides refinements to the pad's measurement. As illustrated in Figure 4.3, the cathode boards are covered with a prepreg layer that protects the strips and pads from damage, upon which a resistive carbon coating is sprayed. The prepreg is a layer of uncured FR4, that is glued and pressed to the etched copper surfaces that form the cathodes. This resistive coating has a surface resistance of $100-200 \mathrm{k} \Omega / \square$ with the lower bound corresponding to detectors in the high pseudorapidity region. Beyond protecting the cathode from electric discharge at 


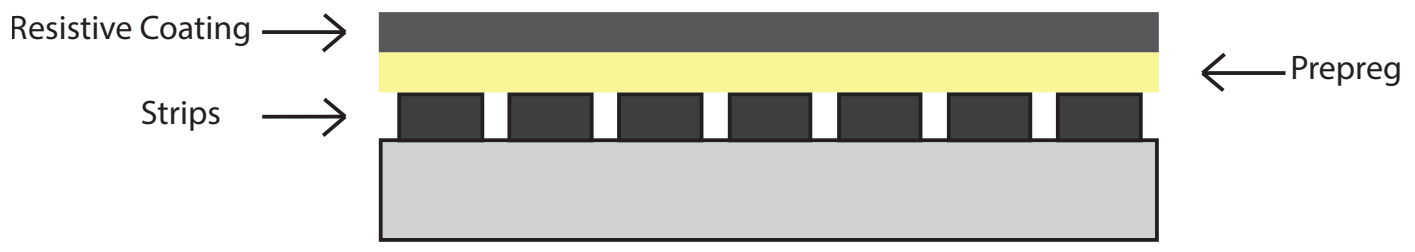

Figure 4.3: Resistive coating is sprayed on to the prepreg layer covering the cathode strips.

high particle rate, the resistive layer improves the spatial resolution of the strips [32]. The gap produced between the strips and the resistive layer due to the prepreg creates a capacitive coupling between the two surfaces. The charge induced on the surface of the resistive layer will follow a bell-shaped distribution and the $R C$ time constant of this coupling will control the spread of the charge density, as mentioned previously in Section 3.3. The $R C$ time constant is one of the tunable parameters in the construction of the sTGC. Typically, the resolution of the cathode strips would be the strip width divided by $\sqrt{12}$ giving a resolution on the order of millimetre's. To achieve the $100 \mu \mathrm{m}$ resolution design requirements, the resistive layer controls the spread of the charge distribution with the peak value corresponding to the location of the avalanche within the detector.

At the LHC, the protons beams cross one another every $25 \mathrm{~ns}$ at the IP. The use of n-pentane- $\mathrm{CO}_{2}$ as the fill gas ensures the ionized electrons drift towards the wire within this time frame. The drift velocity as a function of electric field in this mixture is given in Figure 4.4a. Figure 4.4b is the distribution of the arrival time of the electron clusters to the anode wires in the sTGC after several events. The majority of the distribution shows that the clusters arrive within $25 \mathrm{~ns}$. This ensures that any events that occur within the sTGC are accurately measured before another event occurs. In the NSW the residual magnetic field produced by the toroid and solenoid magnets is rather small (less than $0.4 \mathrm{~T}$ ) and the variation of the drift velocity due to a Lorentz force is negligible. 


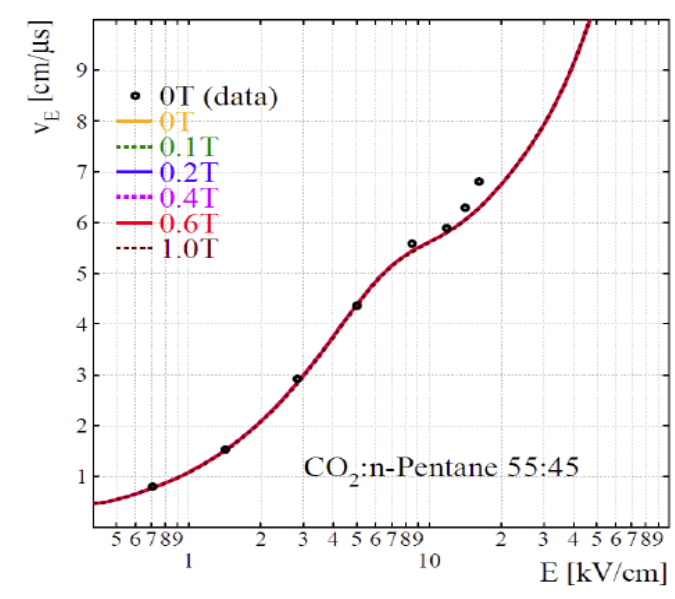

(a)

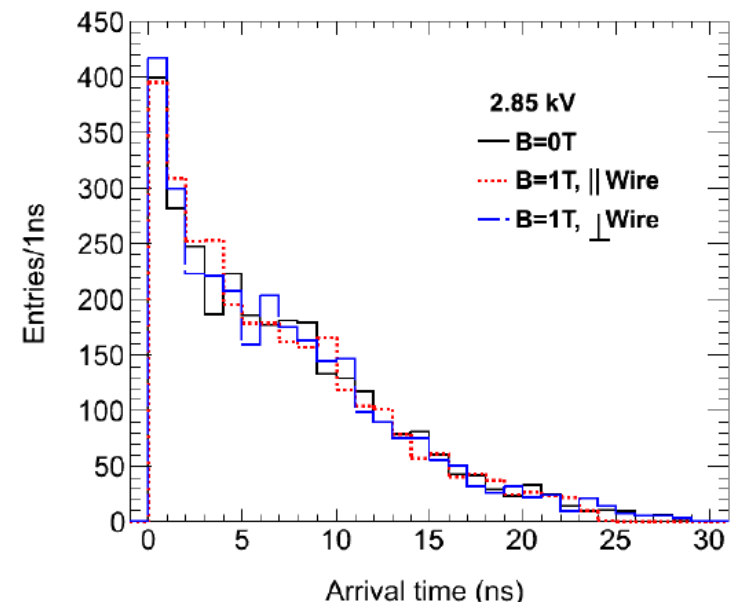

(b)

Figure 4.4: (a) Simulation of drift velocity as a function of electric field in the sTGC at various magnetic fields. (b) Simulation of the time of arrival of electron clusters to the sTGC anode wires held at $2.85 \mathrm{kV}$ in n-pentane- $\mathrm{CO}_{2}$ in the presence of various magnetic fields [14].

All wires, strips and pads are connected to an Application-Specific Integrated Circuit (ASIC) chip named the VMM that monitors the current through the electrodes. The VMM uses a time-over-threshold to determine when a signal should be read out. To ensure background noise is not recorded, the VMM has an adjustable threshold set above the noise within the sTGC. The threshold of the VMM is set to $180 \mathrm{mV}$. Used along side the pad grouping logic, once a signal is over the threshold it is readout using a 10-bit flash Analogue-to-Digital Converter (ADC) [14]. The VMM ADC outputs a value in terms of Peak Detector Output (PDO) which is proportional to the total charge collected on the electrodes. The signal is read out from all detector elements for use in the offline track reconstruction except for the wires in the high pseudorapidity region of the NSW as mentioned previously. 


\subsection{Simulation}

The detailed simulation of the sTGC is performed using the Garfield gas detector simulation software which utilizes the Heed and Magboltz packages to simulate the ionization and transport properties of the clusters in n-pentane- $\mathrm{CO}_{2}$ [33]. The software uses the nearly exact Boundary Element Method (BEM) to determine the electric field produced by the wires in the gap between the cathodes [34]. The BEM is also used to determine the weighted potential of each readout electrode. When a charged particle passes through the detector, Garfield records the signal produced by the motion of the ionization clusters using Ramo's theorem.

A small section of the detector comprised of five wires, seven strips, and one pad is defined to make up the geometry of the sTGC within Garfield and is presented in Figure 4.5. The wires are $50 \mu \mathrm{m}$ in diameter and $22.4 \mathrm{~mm}$ long, the dimensions of the strips are $10.8 \times 3.2 \mathrm{~mm}^{2}$, and the pad is $10.8 \times 22.4 \mathrm{~mm}^{2}$. The potential of the wires is set to $2.85 \mathrm{kV}$ and they are located $1.4 \mathrm{~mm}$ from either cathode with a centre-tocentre spacing of $1.8 \mathrm{~mm}$. The volume between the cathode planes is filled with the 45:55 mixture of n-pentane: $\mathrm{CO}_{2}$. To measure the detector response in the simulation, single muon tracks with energy $4.0 \mathrm{GeV}$ are simulated passing through the detector from $z=-0.28 \mathrm{~cm}$ to $z=0.0 \mathrm{~cm}$. Multiple simulations are carried out with tracks randomly positioned between $0.46 \mathrm{~cm}<x<0.62 \mathrm{~cm}$ and $0.64 \mathrm{~cm}<y<1.60 \mathrm{~cm}$. In the $x z$-plane, this is the region around the centre wire within half the wire spacing away from it on the $x$-axis. In the $y z$-plane, this is the region spanned from the bottom of strip 3 to the top of strip 5 on the $y$-axis. All muon tracks enter the simulated sTGC at normal incidence because the test beam data is produced the same way.

Once the simulation is completed Garfield stores the information of the induced signals on each electrode individually. Using Ramo's theorem Garfield determines the current as a function of time that flows through the electrodes, however, Garfield 


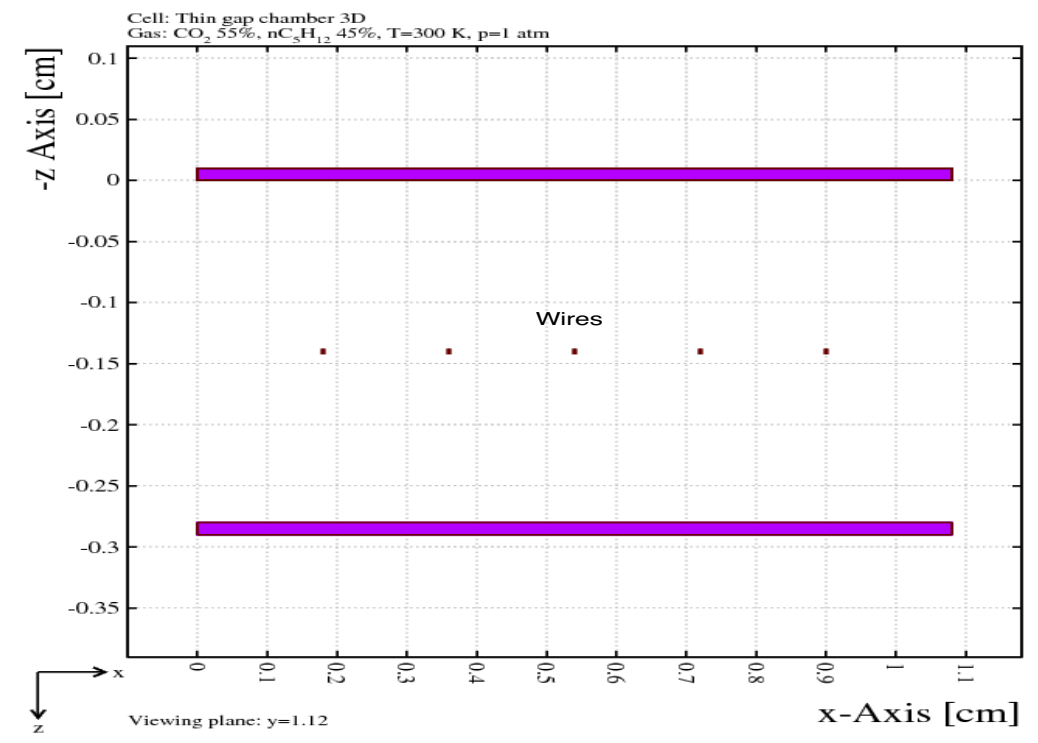

(a)

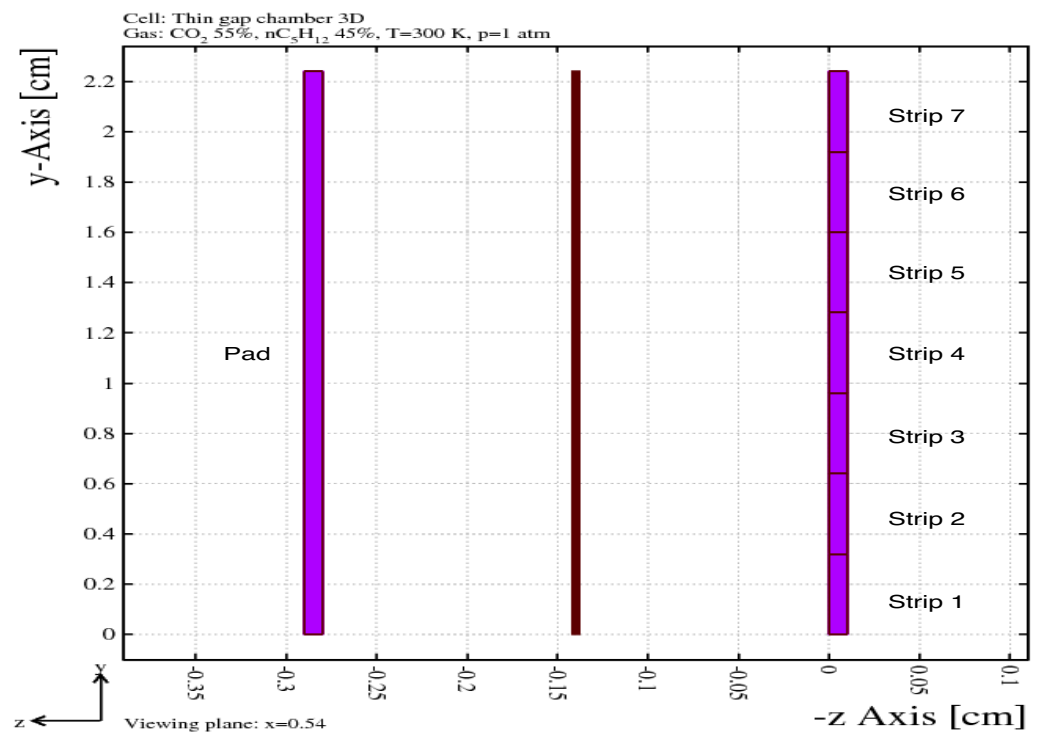

(b)

Figure 4.5: (a) Cross-sectional view in the $x z$-plane showing the wires between the cathodes. Wires are into and out of the page. (b) Cross-sectional view in the $y z$-plane showing the individual strips on one cathode and the pad opposite it. Again, strips/pads are into and out of the page.

cannot account for the response of the resistive layer. The result produced represents the current that flows on the electrode when there is no resistive layer. The general response of a gas detector is thus modelled through consecutive convolution of the 
original signal. Figure 4.6a highlights the process to obtain the final signal on the wires starting from the initial ionization within the detector. Since the avalanche on the wire is not affected by the resistive layer, the only convolution to be considered is the raw wire signal with the shaping function of the electronics. Furthermore, the procedure to simulate the signal from the strips is outlined in Figure 4.6b. Since the

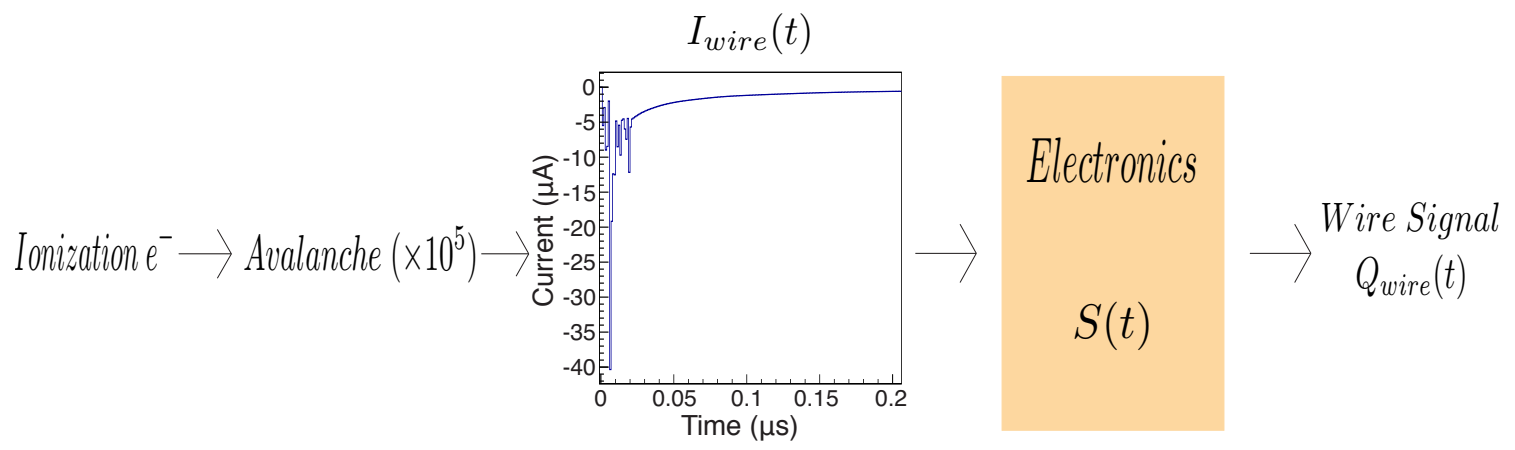

(a)

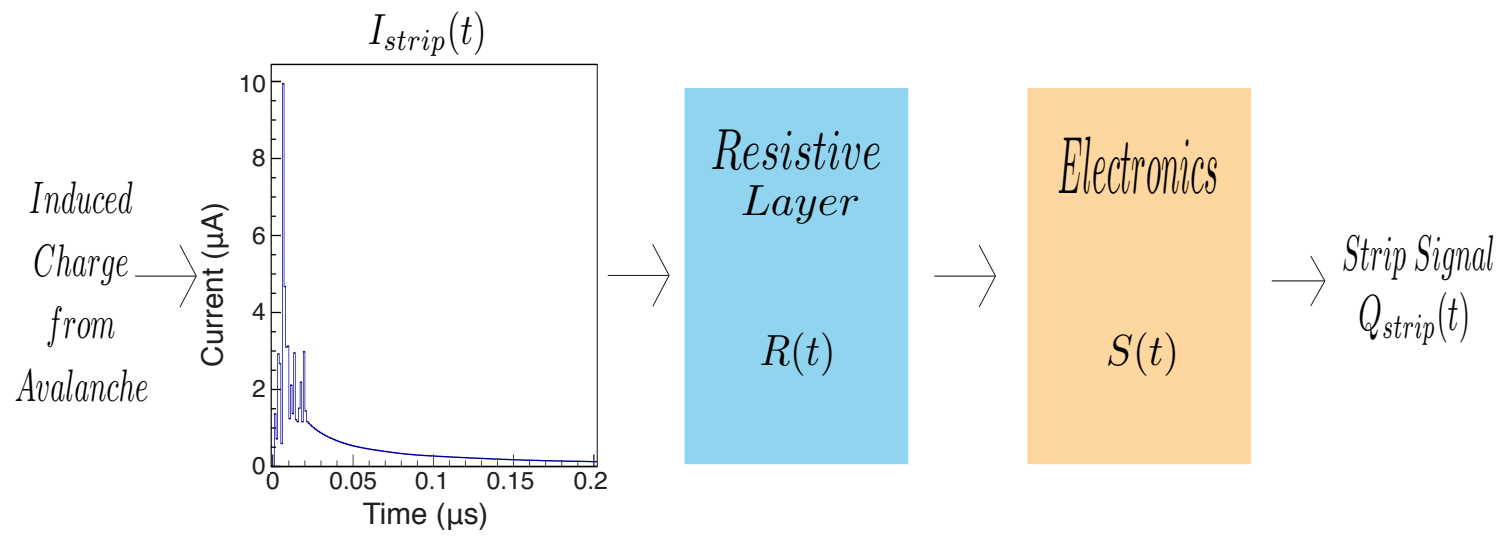

(b)

Figure 4.6: (a) The steps to simulate the signal on the wires in the sTGC. $I_{\text {wire }}(t)$ corresponds to the raw current signal from the wire, $S(t)$ is the shaping function of the electronics, and $Q_{\text {wire }}(t)$ is the final shaped pulse from the wire. (b) The steps to simulate the signal on the strips in the sTGC. $I_{\text {strip }}(t)$ corresponds to the raw current signal from the strip where its amplitude is driven by Ramo's theorem, $R(t)$ is the response function of the resistive layer, $S(t)$ is the shaping function of the electronics, and $Q_{\text {strip }}(t)$ is the final shaped pulse from the strip. 
cathodes are covered with the resistive coating a convolution is first conducted to account for the response of the detector to charge that flows on the resistive layer. Once the resistive layer is considered the response to the electronics can then be considered similarly to the wire. The following sections will show how the response of the readout electronics and resistive layer are taken into account in the signal formation on the sTGC electrodes.

\subsection{Anode Signal}

\subsubsection{Time of Arrival of the Electron Clusters}

When ionization occurs in the sTGC the electrons drift toward the anode wires following the electric field lines. The wire nearest the ionization cluster will collect the drifting electrons on its surface and the motion of the electrons induces charge on the neighbouring electrodes in accordance with Ramo's theorem. The time of arrival of the electron clusters to the wire is determined from the signal corresponding to the one that collects the electrons. It can be used with the drift velocity of the gas to determine where the original ionization event occurred. Figure 4.7 shows the raw signals produced in Garfield when a single muon passes through the sTGC (a) $150 \mu \mathrm{m}$ and (b) $850 \mu \mathrm{m}$ away from an anode wire. It is evident that the location of an ionization event within the detector can be observed from the timing of the wire signal.

The arrival time of the first electron clusters in the simulation are measured to ensure the sTGC is modelled correctly. The measurement involves using the raw signal from the wires produced by Garfield. For each event the wire that collects the electrons is determined from the negative polarity of its signal, from this the histogram of the signal is analyzed to find the first non-zero bin which corresponds to when the first electrons are collected. Figure 4.8 shows a zoomed in version of the 


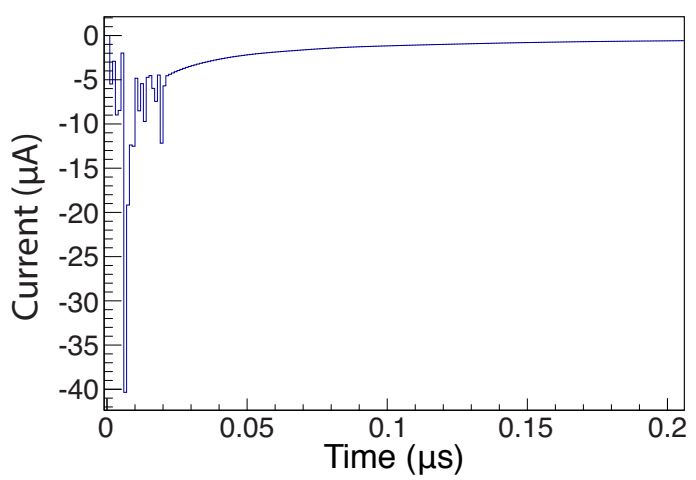

(a)

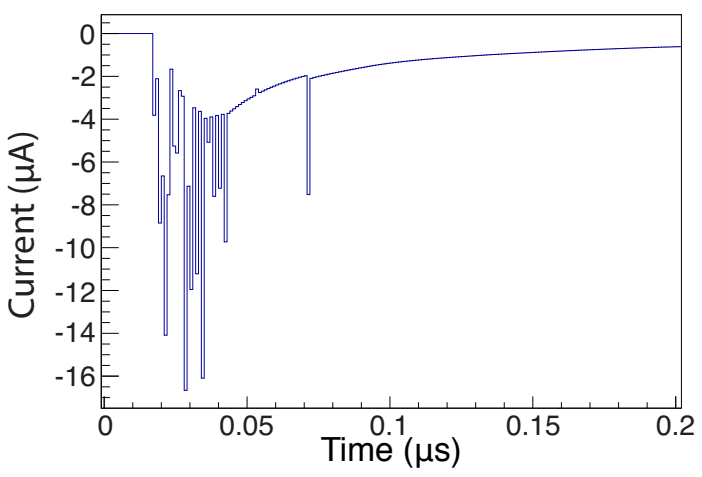

(b)

Figure 4.7: (a) Simulation of the raw signal produced on the wire that collects the electron clusters for a single muon track passing $150 \mu \mathrm{m}$ away from the wire. (b) Simulation of the signal produced on the wire that collects the electron clusters for a single muon track passing $850 \mathrm{\mu m}$ from wire. Both are raw signals before any signal processing is done. One can see individual electron clusters arriving at the anode wires.

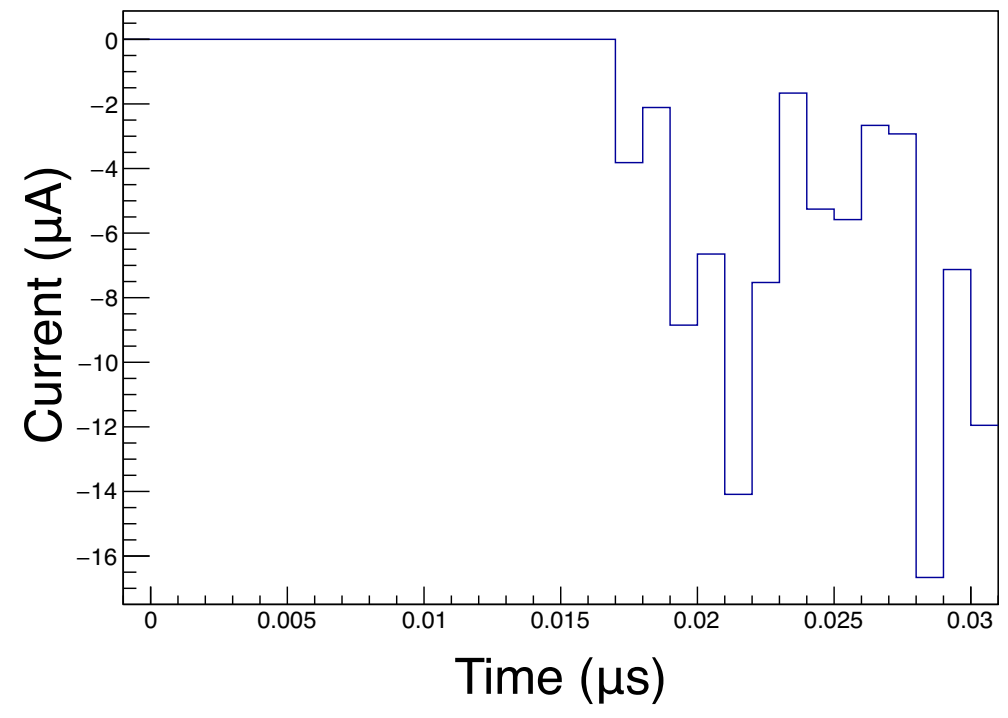

Figure 4.8: Zoomed in example of the raw signal for the simulated single muon passing through the sTGC $150 \mu \mathrm{m}$ away from the wire. The cluster appears to arrive at the wire in approximately $16 \mathrm{~ns}$.

simulated signal in Figure 4.7a where a single muon track passed $150 \mu \mathrm{m}$ away from the wire. The first non-zero bin in this example is found to be at $17 \mathrm{~ns}$; therefore, 
the first electron cluster arrived to the wire $16 \mathrm{~ns}$ after the passing of the muon. The arrival time of the cluster to the wire is determined by the position of the bin before the first non-zero bin. The bin number would correspond to how many nanoseconds elapsed before the electron cluster arrived.

This procedure is repeated to determine the time of arrival for each simulated event in the sTGC to obtain an overall distribution for the simulations. Highlighted in Figure 4.9a is the resulting distribution for the time of arrival of electron clusters to the wire. In the simulations the clusters from almost all events arrive within the $25 \mathrm{~ns}$ time frame with the majority arriving in $5 \mathrm{~ns}$ or less. The distribution in Figure 4.9a is in agreement with the known distribution of the NSW Technical Design Report for n-pentane- $\mathrm{CO}_{2}$ presented in Figure 4.9b. This result confirms and validates that the ionization and transport properties of the electron clusters are accurately represented in the simulation of the sTGC presented here.

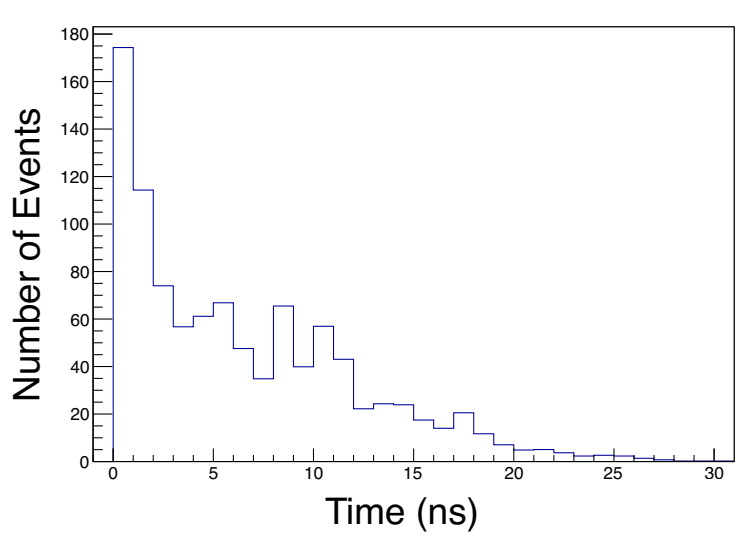

(a)

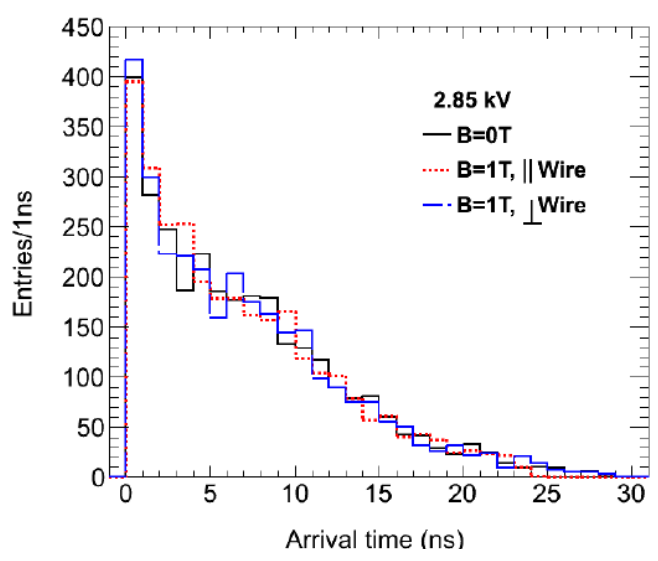

(b)

Figure 4.9: (a) Distribution of the time of arrival for electron clusters to the wire for all simulated single muon track events performed during this work. (b) The published results for the known time of arrival distribution of the sTGC [14]. 


\subsubsection{Anode Electronics Response}

All sTGC electrodes (pads, strips, and wires) are connected to the VMM ASIC chip, however, only the signals produced by the wires can solely consider the detector response to the electronics as explained in Section 4.2. The VMM uses a timeover-threshold of the signal to provide an approximate measure of the charge on the electrodes. Utilizing the time-over-threshold slows the original signal and shifts its peak in accordance with the peaking time of the VMM shaping function. This shaping function is specific to the device itself and is of order 3 with one real pole and two complex-conjugate poles, it is given by

$$
S(t)=\alpha^{3}\left|p_{0}\right|\left|p_{1}\right|^{2}\left[K_{0} e^{-t p_{0}}+2\left|K_{1}\right| e^{-t \operatorname{Re}\left(p_{1}\right)} \cos \left(-t \operatorname{Im}\left(p_{1}\right)+\angle K_{1}\right)\right] .
$$

In Equation $4.1 \alpha$ is proportional to the peaking time, $t_{\text {peak }}$, of the VMM by

$$
\alpha=1.5 t_{\text {peak }},
$$

$p_{0}$ is the real pole

$$
p_{0}=\frac{1.263}{\alpha}
$$

$p_{1}$ gives the two complex-conjugate poles as

$$
p_{1}=\frac{1.149-0.786 i}{\alpha}
$$

and the constants $K_{0}=1.584$ and $K_{1}=-0.792-0.115 i$.

The output of the sTGC is proportional to the charge on the specific electrode. To model the response of the VMM in the simulations, the signal current before readout is convoluted with the VMM shaping function. That is, the total charge on the wire during a single event is given by

$$
Q_{\text {wire }}(t)=\int I_{\text {wire }}(t) \otimes S(t) d t
$$


where $Q_{\text {wire }}(t)$ is the total charge on the wire as a function of time, $I_{\text {wire }}(t)$ is the raw current signal from the wire, and $S(t)$ is the VMM shaping function. The convolution of the wire signal from Garfield and the VMM shaping function with a peaking time of $50 \mathrm{~ns}$ is performed and depicted in Figure 4.10. The peaking time of $50 \mathrm{~ns}$ is used since the simulations are to be compared with test beam data taken with that value. The convolution of the two histograms is performed as a discrete sum of the product of the bin values weighted by the bin width. The result gives the final charge as a function of time for that respective wire.
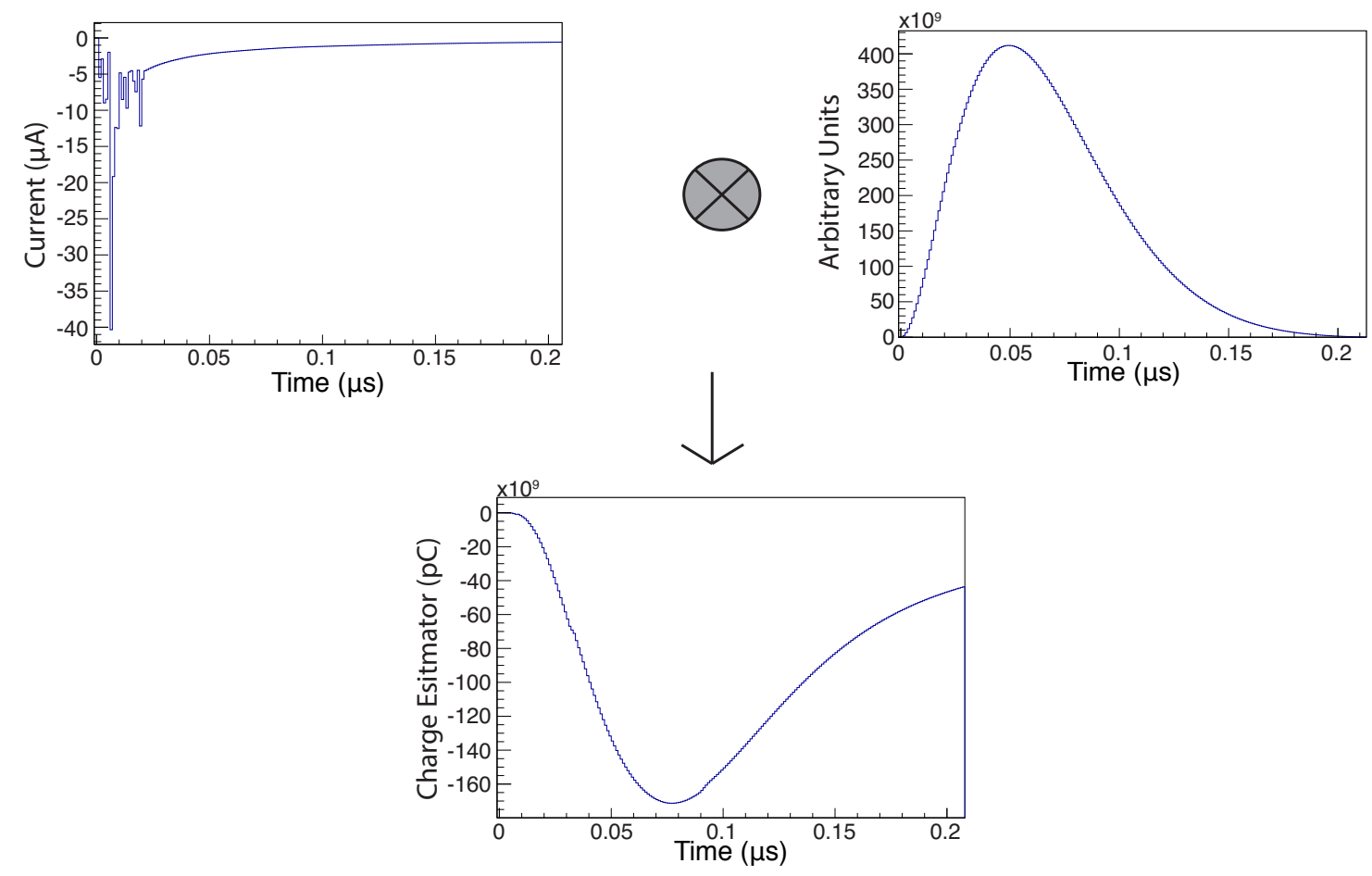

Figure 4.10: The convolution of the raw wire signal with the VMM shaping function with a peaking time of $50 \mathrm{~ns}$. The result gives the total charge on the wire as a function of time. 


\subsection{Cathode Signal}

\subsubsection{Signal Production on Strips}

As a muon traverses a sTGC chamber it deposits energy in the n-pentane- $\mathrm{CO}_{2}$ in accordance with Equation 3.1 and this deposition produces ionization clusters in the gas. The process that a single electron/ion pair will undergo after ionization is depicted in Figure 4.11. In reality several pairs will be produced in a single cluster, however, for simplicity the single pair case is considered to explain the creation of the signal on the cathodes. In the presence of the electric field produced by the wires, the electrons and ions drift towards their respective electrodes following the field lines. Due to their smaller size, the electrons have a much greater drift velocity in the gas and can attain a higher energy. In the high electric field region of the wires, within a few wire radii, the electrons have gained enough energy to further liberate electrons in the gas molecules producing secondary ionization. This is shown in Figure 4.11b. The gas gain of n-pentane- $\mathrm{CO}_{2}$ produces about $10^{5}$ electrons in the avalanche at the wire. The rapid production of electrons drifting towards the wire induces charge in accordance with Ramo's theorem on the surface of the cathode, illustrated in Figure 4.11c. The slower moving ions contribute to the signal produced at the cathodes, however, the resistive layer and VMM mitigate this slow contribution to the final signal read out. The final VMM signal read out from the strips will give a value proportional to the total charge that is collected on its surface.

\subsubsection{Signal Processing}

To simulate the signals that are produced by the cathodes strips, first the response of the resistive layer is considered. The charge dispersion over the resistive layer is simulated using a response function as presented in Chapter 3 for a surface with a 


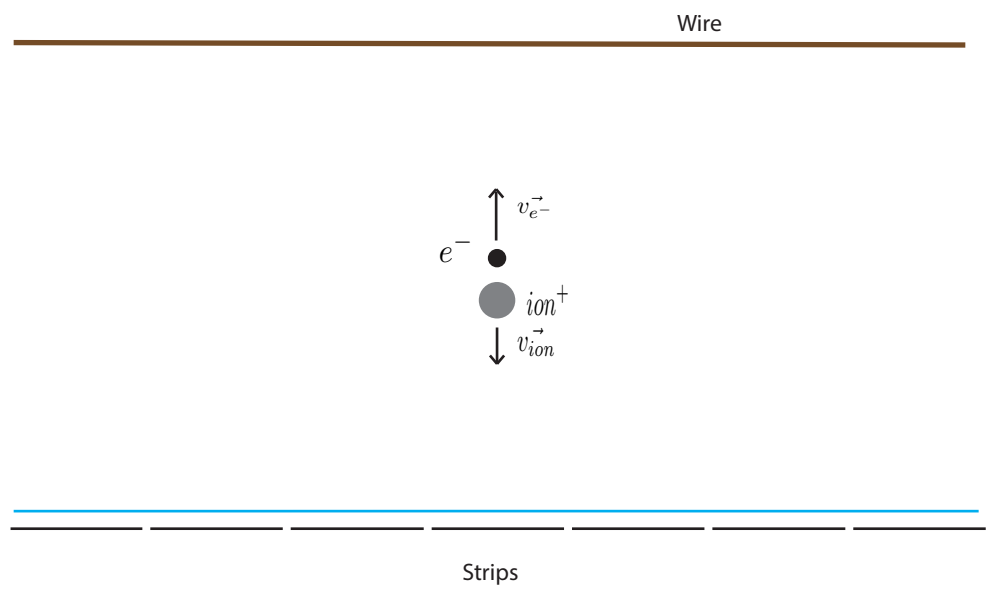

(a)
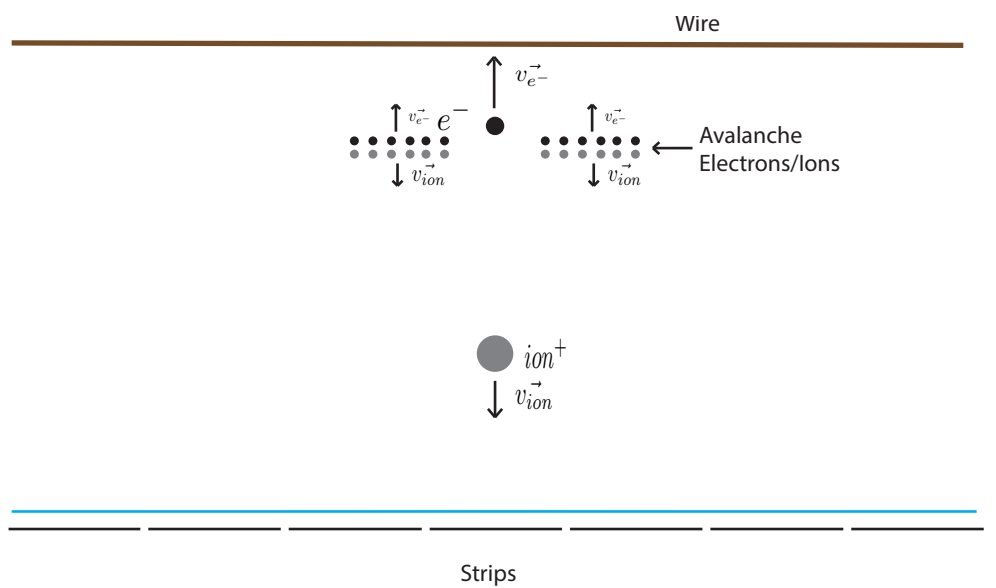

(b)

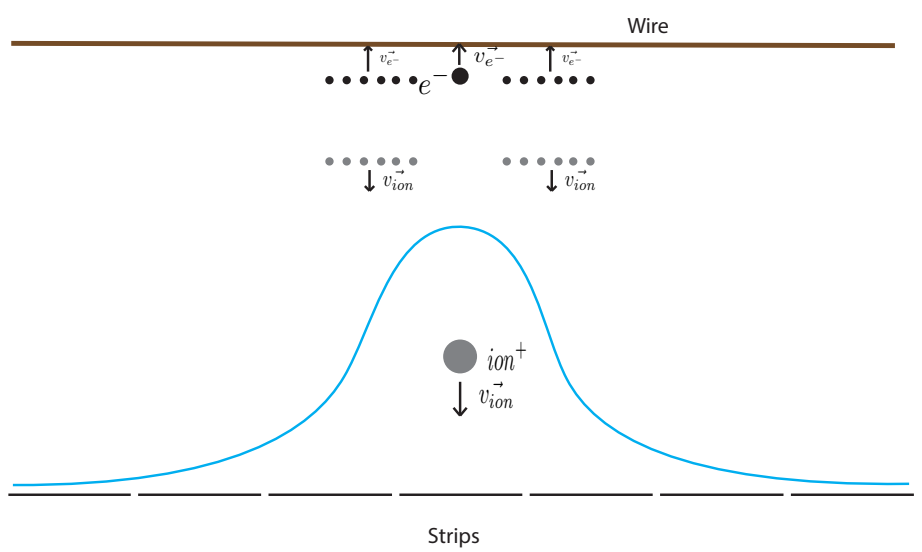

(c)

Figure 4.11: (a) Single ionization event, electrons begin to drift towards their respective electrodes. (b) Once electrons are within a few wire radii of the wire they have enough energy to produce secondary ionization electrons in an electron avalanche.(c) The rapid production of electrons at the wire induces charge on the strips. The charge density will follow a Gaussian-like distribution over the strips. The blue line depicts the charge distribution when the relative amplitudes of the strip signals are plotted. 
ground on one side. The response function is given by

$$
R(t)=\frac{1}{\pi}\left[\tan ^{-1}\left(\frac{y_{h i g h}}{2 \sqrt{h t}}\right)-\tan ^{-1}\left(\frac{y_{\text {low }}}{2 \sqrt{h t}}\right)\right],
$$

where $y_{\text {high }}$ and $y_{\text {low }}$ define the two edges of the strip the charge flows to with respect to the origin $(x, y)=(0,0)$ of where the point-charge is produced (or induced). To determine the charge that flows from strip $i$ to strip $j$ the raw signal of strip $i$ is convoluted with the response function corresponding to strip $j$ 's position from strip $i$. Garfield produces a signal in terms of current, therefore, the convolution with the response function gives an integral with respect to time resulting in the charge
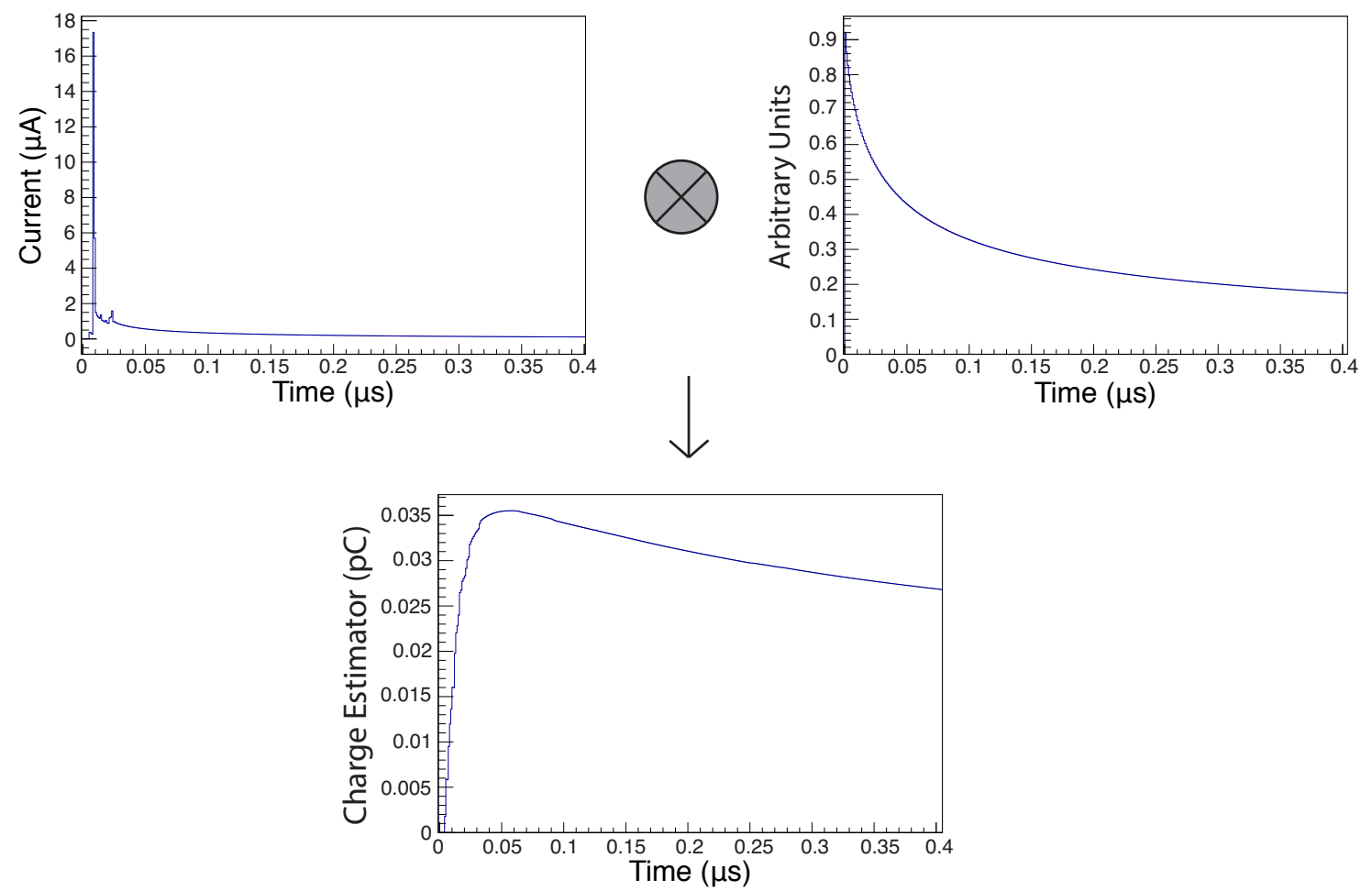

Figure 4.12: Raw signal from strip 4 (top-left) convoluted with $R_{0}$ (top-right) resulting in the charge that flowed across itself as a function of time (bottom). 
which is shown in Equation 4.7.

$$
Q_{i j}(t)=\int I_{i}(t) \otimes R_{k}(t) d t
$$

where $Q_{i j}$ is the total charge as a function of time that flows from strip $i$ to strip $j$, $I_{i}(t)$ is the raw current signal on strip $i$ produced by Garfield as a function of time, and $R_{k}(t)$ is the response function corresponding to the $k^{t h}$ neighbour of strip $i$ as a function of time where $j=i \pm k$. More simply, strip $j$ will be $k$ strips away from strip $i$. In Appendix A the histograms of the seven response functions corresponding to the seven strips are presented. In Figure 4.12 the raw signal from strip 4 is convoluted with the histogram corresponding to

$$
R_{0}(t)=\frac{1}{\pi}\left[\tan ^{-1}\left(\frac{w / 2}{2 \sqrt{h t}}\right)-\tan ^{-1}\left(\frac{-w / 2}{2 \sqrt{h t}}\right)\right],
$$

where $w$ is the strip width of $3.2 \mathrm{~mm}$. The result gives the charge that remains on the strip from the original induced charge on its surface. Equation 4.8 evaluates the charge over the boundaries $-w / 2$ to $w / 2$, this corresponds to the edges of the individual strip itself. To continue. Figure 4.13 highlights the convolution of strip 4 with the histogram corresponding to

$$
R_{2}(t)=\frac{1}{\pi}\left[\tan ^{-1}\left(\frac{5 w / 2}{2 \sqrt{h t}}\right)-\tan ^{-1}\left(\frac{3 w / 2}{2 \sqrt{h t}}\right)\right] .
$$

The boundaries defined in Equation 4.9 correspond to the second neighbour away from the strip. The result gives the charge that flows from strip 4 to strip 6 , but due to the symmetry of the dispersion this is also equivalent to the charge that flows from 4 to 2 . This procedure was repeated for all strips for each possible neighbour to obtain the total charge contributions of each strip to all the others. 

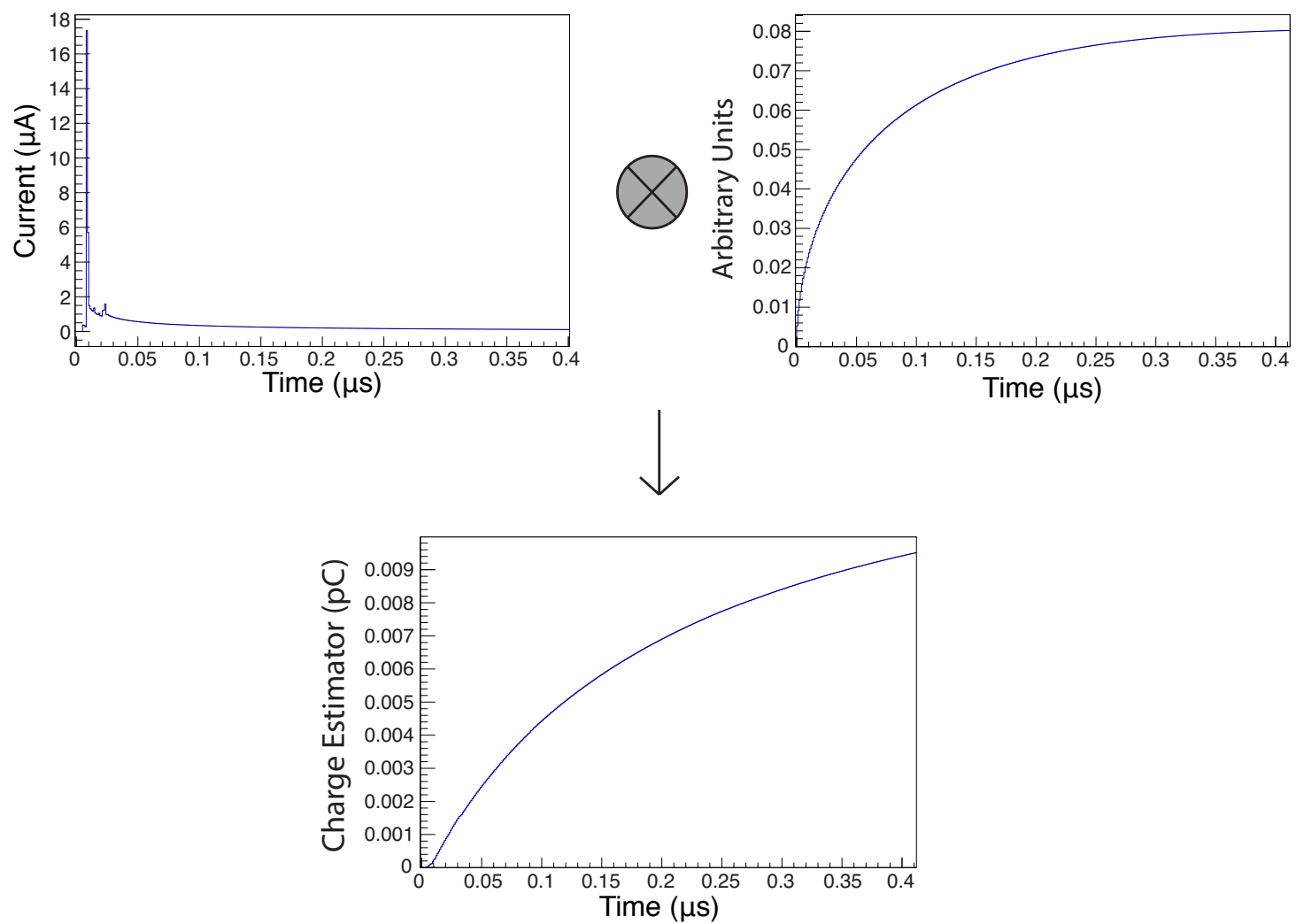

Figure 4.13: Raw signal from strip 4 (top-left) convoluted with $R_{2}$ (top-right) resulting in the charge that flowed from it to its second closest neighbour as a function of time (bottom).

The determination of the total charge as a function of time on an individual strip is performed by summing the contributions of each strip. This is given by

$$
Q_{j}(t)=\sum_{i=1}^{7} Q_{i j}(t),
$$

where $Q_{j}(t)$ is the total charge on strip $j$ as a function of time.

Once the response of the resistive layer is simulated for the strip signals, the effects of the VMM can be modelled similarly to the procedure outlined for the wire. Since the sTGC outputs a signal proportional to the charge on the strips, the derivative of 


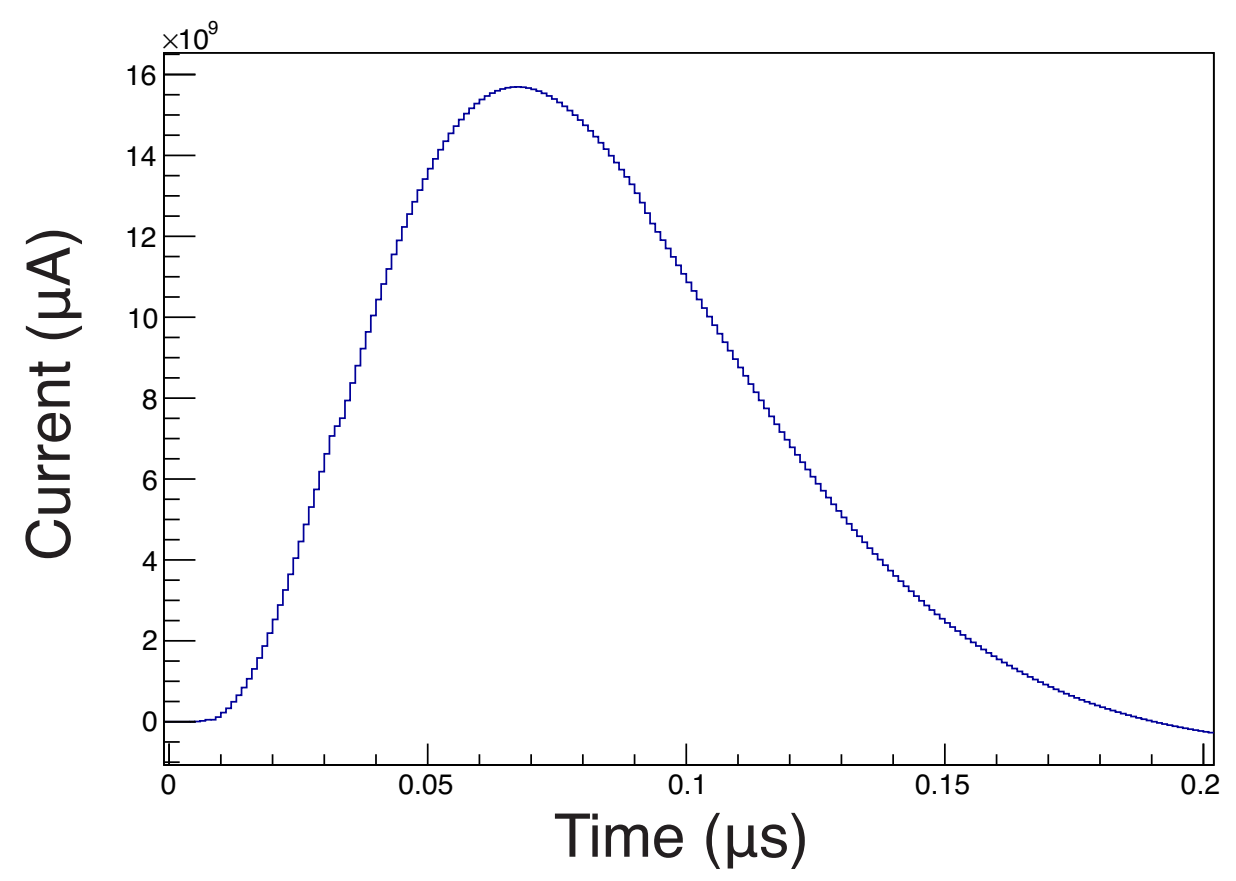

(a)

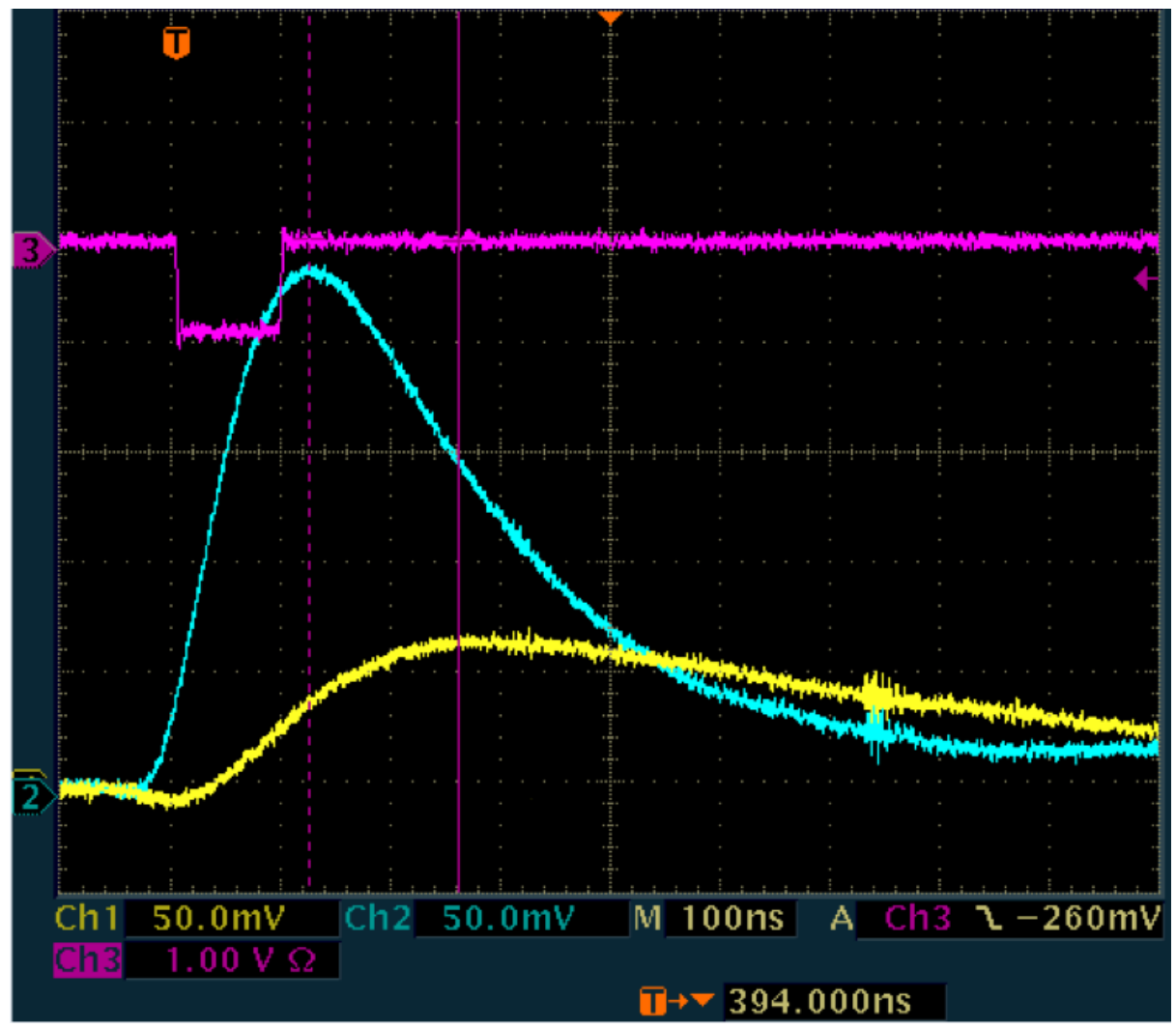

(b)

Figure 4.14: (a) The final signal from strip 4 which corresponds to the central strip for this event. (b) An oscilloscope reading of a muon event during an sTGC test beam at Fermi National Laboratory. The central strip corresponds to the high peaked signal. 
the total summed charge on strip $j$ above is taken yielding

$$
I_{j}^{\prime}(t)=\frac{d}{d t} Q_{j}(t),
$$

where $I_{j}^{\prime}(t)$ is the total current flowing through strip $j$ after considering the resistive layer. The derivative is obtained from the binned histogram of $Q_{j}(t)$ with a $1 \mathrm{~ns}$ bin width. Now the identical procedure to simulating the wire signal is followed by convoluting the total current through the strip with the shaping function of the VMM

$$
Q_{j}^{\prime}(t)=\int I_{j}^{\prime}(t) \otimes S(t) d t .
$$

In Equation $4.12 Q_{j}^{\prime}(t)$ corresponds to the total charge as a function of time on strip $j$ once all detector response is considered. From this the charge on each strip can be obtained to determine the total charge distribution over the strips for a single muon event. Figure 4.14a shows the final result of the signal on strip 4 after considering all detector response. Comparing it to the central strip signal obtained from an sTGC test beam at Fermi National Laboratory (FNAL) that used a 50 ns peaking time its observed that both signals have their peaks shifted by $100 \mathrm{~ns}$ and both have a similar width. These points support that the sTGC strip signals are being accurately simulated. 


\section{Chapter 5}

\section{Track Reconstruction}

\subsection{Cluster Fitting}

The data generated by the sTGC simulations is analyzed to see how well the $y$-coordinate of a muon track can be reconstructed from the charge distribution over the strips. For each muon track, ionization is deposited in the simulated sTGC gap that generates signals on all the readout elements. The signals are thus processed such that the charge estimators (in Coulombs) correspond to the values of the peak of the signals. Figure 5.1 depicts the simulated signals on all the strips, while Figure 5.2 shows the corresponding charge cluster. The simulated data is processed like real data such that the charge estimator can be directly compared to the VMM Peak Detector Output (PDO).

On an event-by-event basis the charge distribution, or cluster, is plotted such that each bin corresponds to the width and position of the strips within the simulated sTGC. The charge cluster follows a bell-shaped distribution, however, there is no analytical solution to model the distribution of the charge. Due to this, the determination of the location where the avalanche occurs in the detector can be done by fitting the charge cluster with a Gaussian as a best approximation of the distribution. 

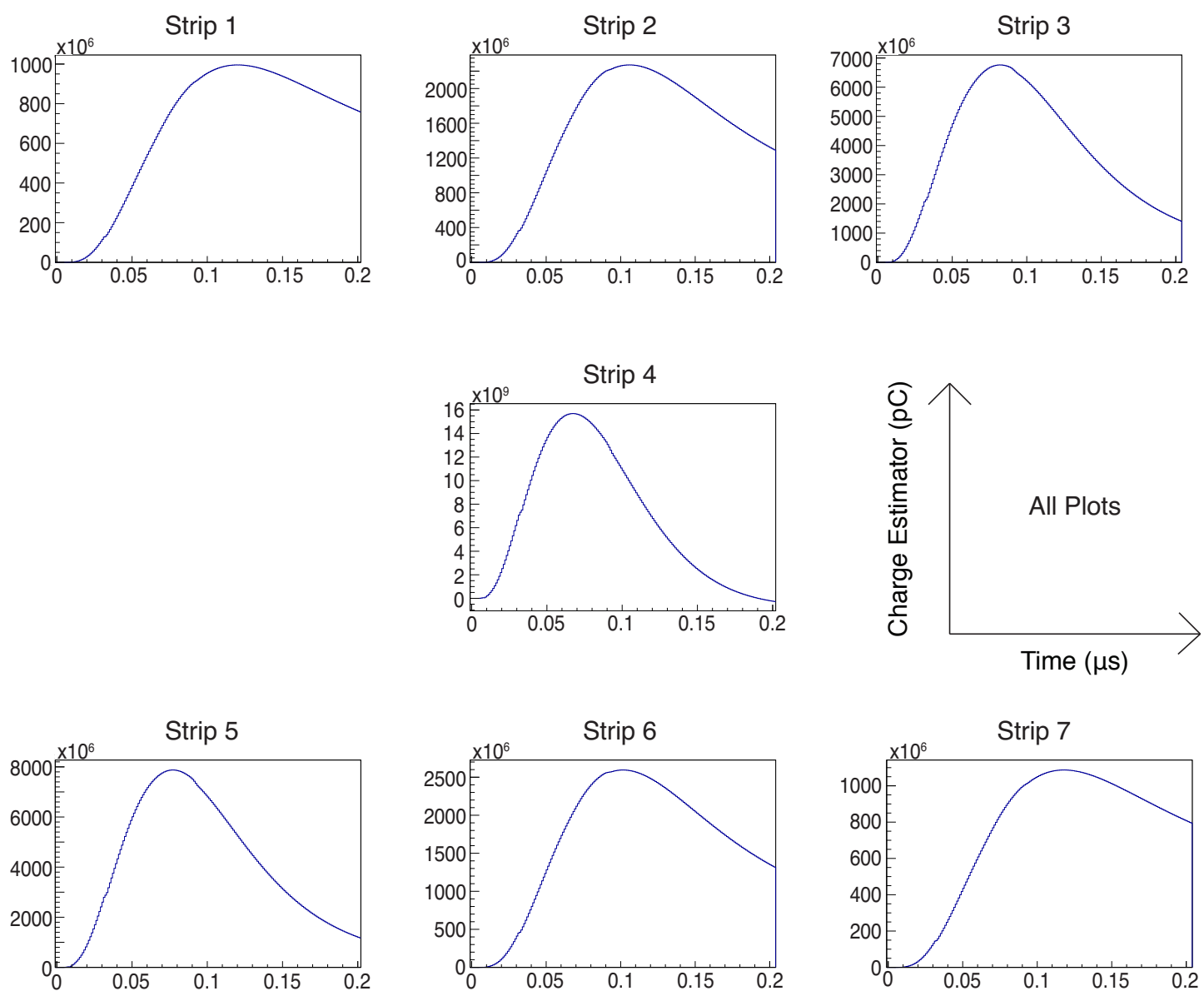

Figure 5.1: The simulated signal produced for each of the seven cathode strips after all detector response is applied. From the signal, the peak value is obtained for an estimator of the maximum charge on each strip to determine the distribution of the charge cluster over the strips.

For the cluster fitting to determine the $y$-coordinate, the fit is conducted over the three central bins of the charge cluster. Figure 5.3 highlights a charge distribution of a single muon event that passes through the region centered over strip 4 . The blue line corresponds to the fit using a Gaussian ranging from the outer edge of strip 3 to the outer edge of strip 5 and the red corresponds to the fit using all seven bins. Qualitatively, the blue line appears to better represent the spread of the distribution for the three central bins. Furthermore, the testing of the sTGC Modulezero prototype at FNAL suggests that a narrow Gaussian is a better description 


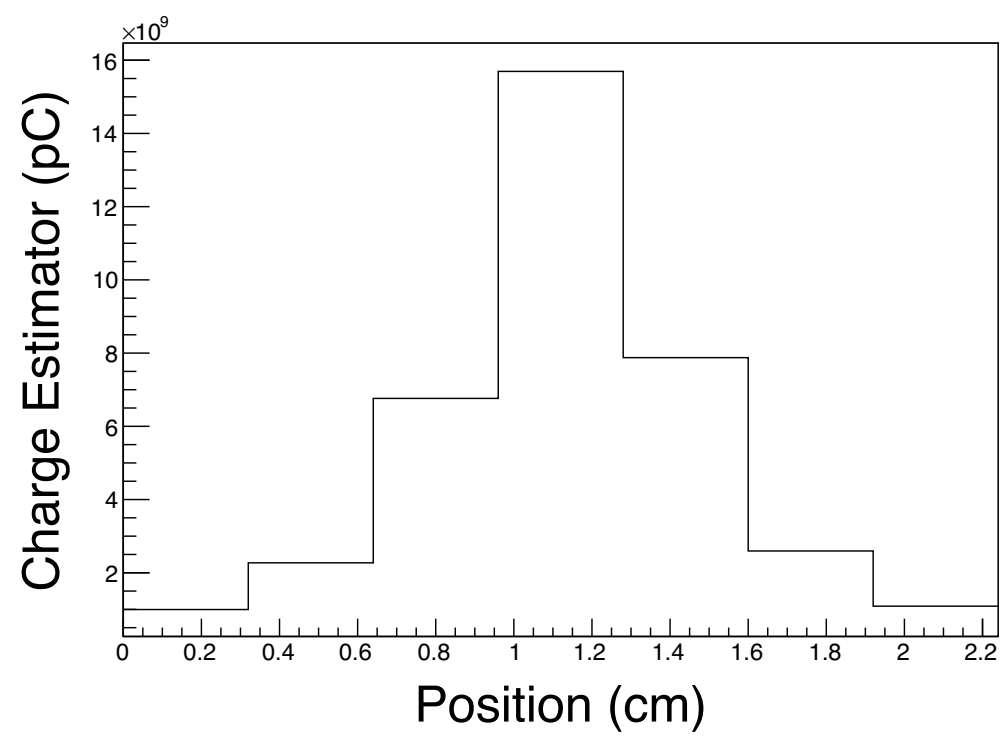

Figure 5.2: The peak values are plotted over their respective strips to show the distribution of the charge cluster.

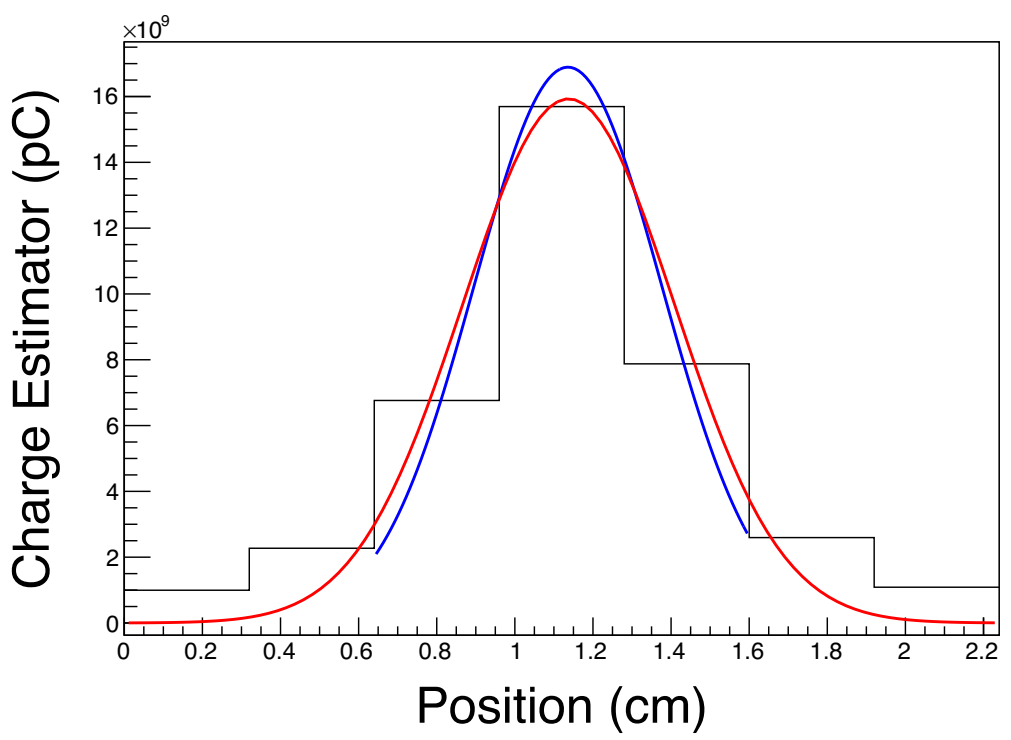

Figure 5.3: Fitting the charge cluster with two separate Gaussian's. The blue line corresponds to the fit over the three central bins, while the red line represents a fit over all seven bins.

of the cluster charge distribution over segmented strips [31]. To quantitatively support this claim, the two fits are tested to compare their shape with the simulated charge 


\begin{tabular}{|l|c|}
\hline & Result (Rel. Units) \\
\hline $\begin{array}{l}\text { Integral of Histogram Over } \\
\text { Three Central Bins }\end{array}$ & $9.706 \times 10^{9}$ \\
\hline $\begin{array}{l}\text { Integral of Fit Over Three } \\
\text { Central Bins }\end{array}$ & $9.706 \times 10^{9}$ \\
\hline $\begin{array}{l}\text { Integral of Histogram Over } \\
\text { All Seven Bins }\end{array}$ & $1.193 \times 10^{10}$ \\
\hline $\begin{array}{l}\text { Integral of Fit Over All } \\
\text { Seven Bins }\end{array}$ & $1.086 \times 10^{10}$ \\
\hline
\end{tabular}

Table 5.1: The results of the shape test comparing the area under the charge distribution for three and seven bins to the area under the respective Gaussian fit lines. The test supports the fit of the three central bins.

cluster. The shape test involves comparing the area under the histogram over the range of both the three central bins and all seven bins with the area under the curve representing the respective fits. In Table 5.1 is a summary of the results of the shape test. As can be seen from the Table, by fitting all seven bins the the fitting function underestimates the area under the distribution by approximately $10 \%$. Conversely, by fitting only the three central bins of the cluster the total area under the Gaussian fit better matches the area under the histogram. These results support the decision to only fit the three central bins to characterize the charge distribution over the strips.

Two different methods are carried out to determine the mean of the charge distribution. The first is a basic approach which involves calculating the weighted mean of the distribution using [22]

$$
\bar{y}=\frac{\sum_{i=1}^{N_{\text {bins }}} y_{i} Y_{i}}{\sum_{i=1}^{N_{\text {bins }}} Y_{i}},
$$

where $y_{i}$ and $Y_{i}$ were the central position and charge of strip $i$, respectively. The 
sum in Equation 5.1 is over the three central bins. As mentioned above, the charge distribution is Gaussian-like so a more sophisticated approach is to fit the three central bins with a Gaussian fit function given by

$$
G(y)=\frac{1}{\sqrt{2 \pi \sigma^{2}}} e^{-\frac{(y-\mu)^{2}}{2 \sigma^{2}}} .
$$

In Equation 5.2, $\mu$ and $\sigma$ are the mean and standard deviation of the Gaussian, respectively, and the two free variables of the fit. From this the mean is obtained and recorded to approximate the location of the avalanche. The means resulting from these two methods are then compared to the true simulated $y$-coordinate of the muon track.

The difference between the true value and the measured value is compared to determine how well the detector can reconstruct the $y$-coordinate. This difference, referred to as the residual, is given by

$$
\text { Residual }=y_{\text {true }}-y_{s T G C}
$$

where $y_{s T G C}$ corresponds to the estimator of the mean measured from the charge cluster, and $y_{\text {true }}$ is the actual $y$-coordinate of the passing muon track.

For each simulated muon event, the true $y$-coordinate of the track is recorded. From this, separate residuals are calculated for each method used to determine $y_{s T G C}$ as explained above. The residuals from the simulations are plotted against both $y_{s T G C}$ and $y_{\text {true }}$ to observe the dependency on the variables. Furthermore, $y_{s T G C}$ is plotted against $y_{\text {true }}$. The simulations are conducted with muon tracks passing through the detector between strip 3 and strip 5, and represent the reconstruction of tracks passing between the middle of strip 3 to the middle of strip 5 . 


\section{$5.2 \quad$ Y-Coordinate Measurement}

Following the analysis outlined above, the $y$-coordinate's of the simulated muon tracks are reconstructed using two different methods to determine the mean of the charge distributions. The plots that are presented in Figure 5.4 give the results obtained using the weighted mean. In Figure 5.4a the residual is plotted as a function of the true $y$-coordinate, $y_{\text {true }}$, of the muon track that is recorded for each event. The range of the residual varies between $0.16 \mathrm{~cm}$ and $-0.16 \mathrm{~cm}$ depending on the track's true position. From the plot it can be seen that tracks that pass directly through the centre of the strips are able to be accurately reconstructed with a zero residual. A change in sign of the residual is seen around the centre of the strips as well at the coordinate corresponding to the point halfway between the strip centre and its edge. The residual is plotted against the calculated weighted mean, $y_{s T G C}$, as shown in Figure 5.4b. Values of the residual again ranged between $0.16 \mathrm{~cm}$ and $-0.16 \mathrm{~cm}$ with it being equivalent to zero for tracks measured to pass through the centre of the strips. However, the residual takes on many different values for the same $y_{s T G C}$ measurement on either side of the strip centres. This is further evident in Figure 5.4c where $y_{s T G C}$ is plotted against $y_{t r u e}$. The red line represents what is expected if the estimator of the reconstructed position is unbiased and could exactly resolve $y_{\text {true }}$. The difference between the red line and the point corresponds to the residual for that point. During the analysis, values of $y_{s T G C}$ appear to remain almost constant for tracks passing through the sTGC off-centre. Two regions of consistency are observed in Figure 5.4c where any tracks that are to the left of the strip's centre are measured to pass through halfway between the centre and its left edge. While tracks that are to the right are measured to pass through halfway between the centre and the its right edge. The divide of the two regions occurs directly at the strip's centre where both the measured and true values are in agreement.

The results obtained from fitting the charge cluster with a Gaussian to obtain 


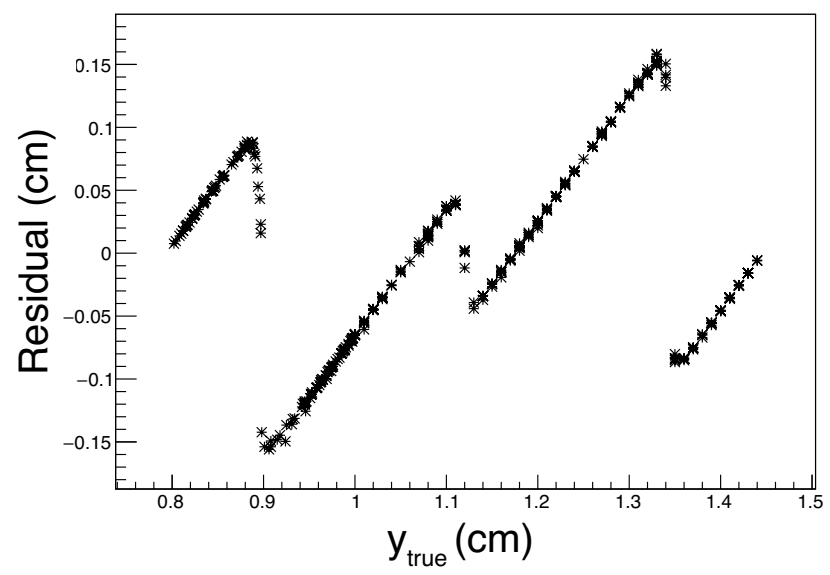

(a)

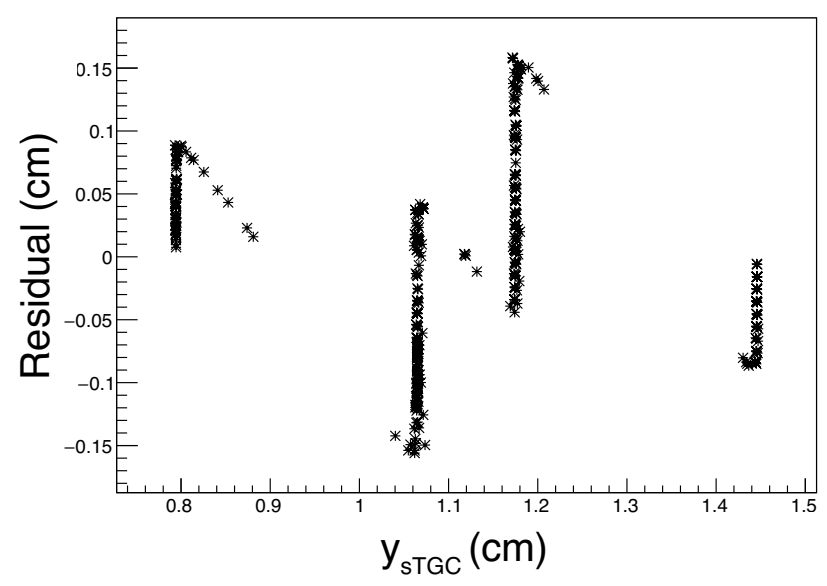

(b)

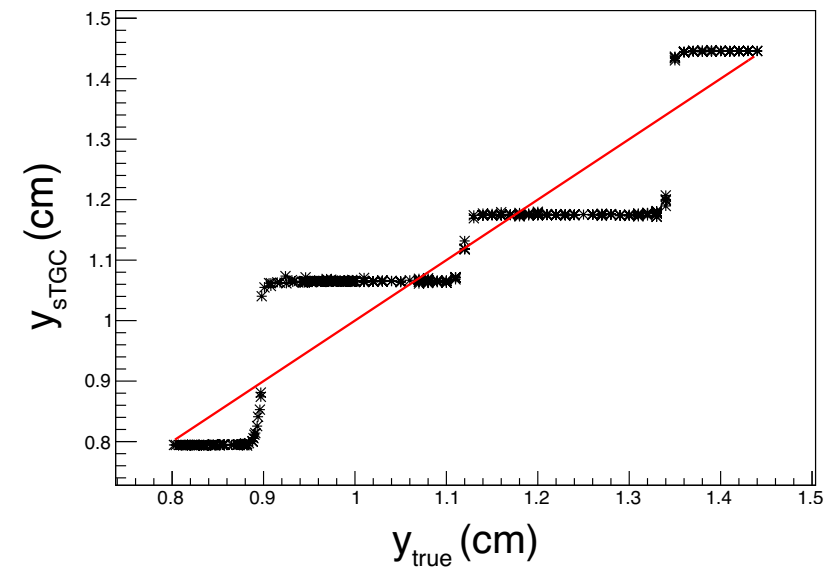

(c)

Figure 5.4: (a) The residual as a function of the true $y$ position of the muon track using the weighted mean. (b) Residual plotted against the reconstructed sTGC coordinate calculated from the weighted mean. (c) The reconstructed sTGC $y$ position plotted as a function the true $y$ position. 


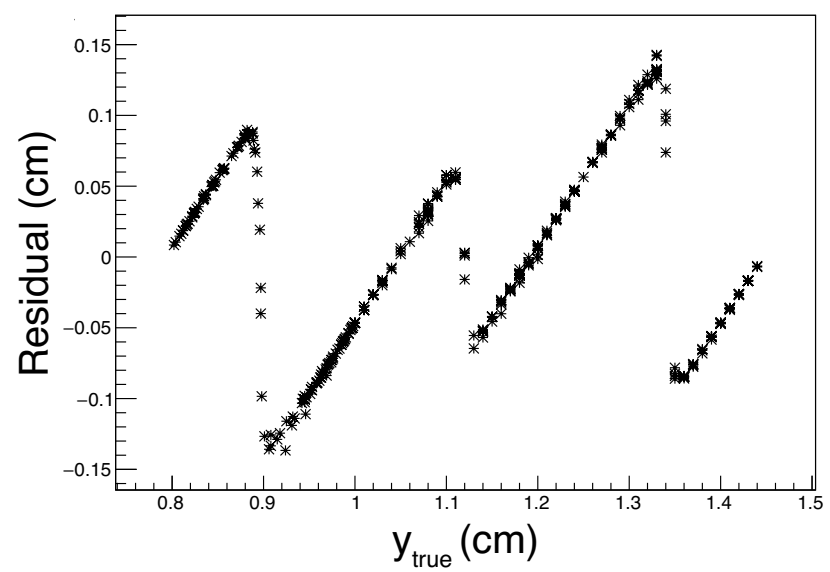

(a)

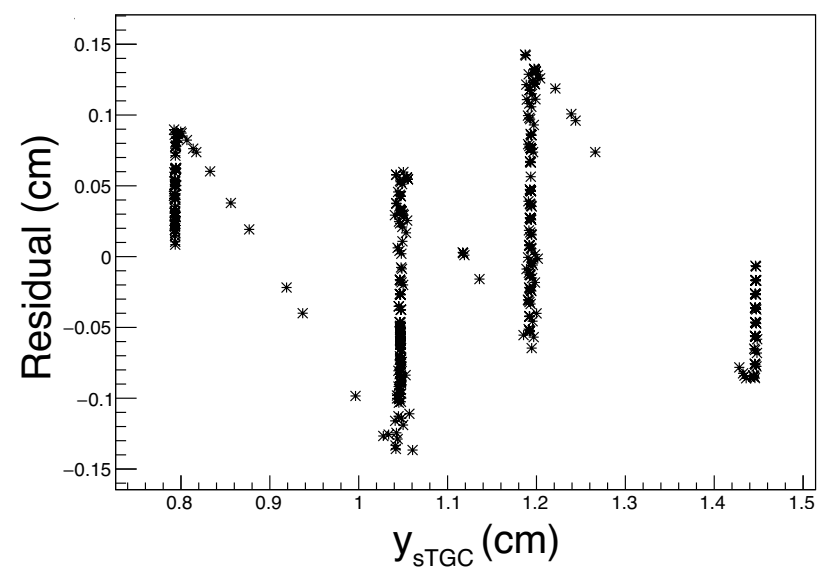

(b)

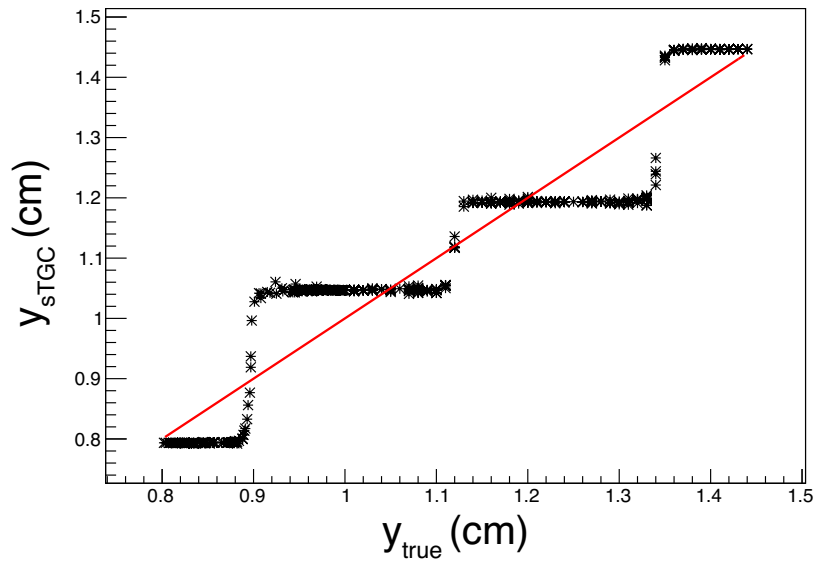

(c)

Figure 5.5: (a) The residual as a function of the true $y$ position of the muon track using a Gaussian fit. (b) Residual plotted against the reconstructed sTGC coordinate calculated obtained from the fit. (c) The reconstructed sTGC $y$ position plotted as a function the true $y$ position. 
$y_{\text {stgc }}$ are plotted in the same form as the weighted mean method above and are given in Figure 5.5. The residual from the Gaussian fit was plotted against $y_{\text {true }}$ and is depicted in Figure 5.5a. Using this method the residual ranges between $0.14 \mathrm{~cm}$ and $-0.14 \mathrm{~cm}$ with an apparent triangular wave structure that forms. Tracks that pass through the centre of the strips are again clearly able to be identified as the residual is zero at these locations. Similar to the results presented using the weighted mean, Figure 5.5b shows the residual took on a range of values for similar measurements of $y_{s T G C}$ for tracks that are not directly over the centre of the strips. A potential cause of this is observed in Figure 5.5c where the measured values of $y_{s T G C}$ using the Gaussian fit are plotted against $y_{\text {true }}$. The red line represents as before the case where the sTGC has ideal resolution. Again it is observed that for tracks that pass even slightly off-centre, their measured position is located approximately halfway between the centre and the respective edge of the strip depending if it passes to the left or right of centre. The cause of this is unknown and could be an artifact of the techniques used for reconstruction.

Further investigation into the reconstruction of the $y$-coordinate needs to be conducted to resolve why the measured value of $y_{s T G C}$ remains relatively constant for a wide a range of off-centre $y_{\text {true }}$ values. Since the bin width is restricted to the width of the strips it can not be adjusted to try and reconcile this issue, therefore other techniques must be considered.

In summary, the simulation can be used to correct for possible bias in the reconstruction of cluster charge associated with hits along a muon track. The ultimate goal is to improve the ability of the NSW to reconstruct muons during the high-luminosity phase of the LHC. 


\section{Chapter 6}

\section{RC Time Constant}

\subsection{Analysis}

In the simulation of the sTGC, one parameter that can be adjusted is the value of the $R C$ time constant due to the capacitive coupling between the resistive layer and the strips. Ultimately, the surface resistivity and the thickness of the prepreg layer dictate how the charge will spread and sets the width of the charge cluster over the sTGC strips. The effects of the $R C$ value on charge dispersion is illustrated in Figure 6.1 where it is seen that a higher $R C$ value results in most of the charge being localized to the central strip. When the value of $R C$ is lower the charge flows more freely over the resistive layer.

The goal of the analysis is to validate the $R C$ time constant to be used in the simulation agrees with the construction parameters of the sTGC tested in a test beam at CERN. The determination of the appropriate time constant is achieved by comparing the simulations to sTGC test beam data provided by the ATLAS Muon Group for which the $R C$ constant is known for the tested detector. The sTGC resistive layer has a $200 \mathrm{k} \Omega$ surface resistance with a prepreg/resistive layer thickness of $200 \mu \mathrm{m}$ resulting in a time constant per unit area of $40 \mathrm{~ns} / \mathrm{mm}^{2}$. The type of sTGC 


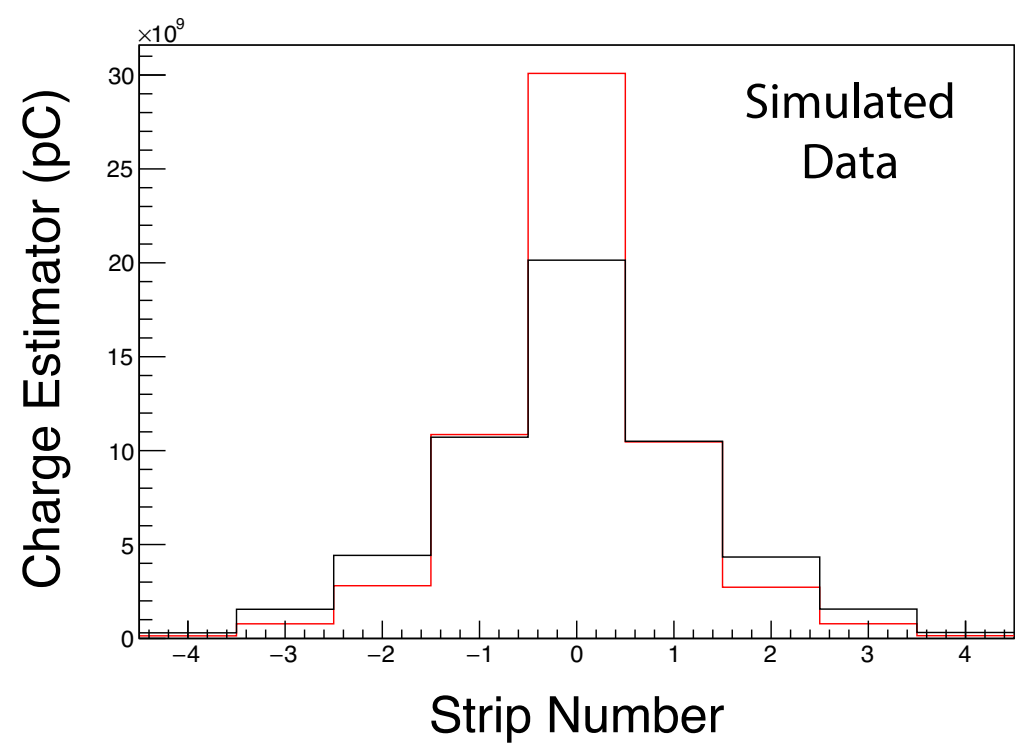

Figure 6.1: The effect of $R C$ on charge dispersion over the strips. The black line corresponds to a cluster using an $R C$ value of $21 \mathrm{~ns} / \mathrm{mm}^{2}$ while the red line corresponds to an $R C$ of $120 \mathrm{~ns} / \mathrm{mm}^{2}$

used for the test beam run is a complete QS2 quadruplet.

The test beam data contains the results from over one million single muon track events with energy of $150 \mathrm{GeV}$. From each event the PDO value of each strip is obtained. The central strip is determined from the maximal PDO value of all the strips. On a per event basis, the values of the central strip, first left neighbour, first right neighbour, and so on moving outward from the central strip are saved in an array with each element corresponding to their position relative to the central strip. The array is summed over all events and then divided by the number of events to determine an average charge distribution over the strips in each layer. The analysis looks at the average distribution from three out of the four layers of the quadruplet. The results from the second layer of the sTGC are omitted since the layer appears to have malfunctioned. The average charge distribution of the strips for layers 1,3 , and 4 from the test beam data are shown in Figure 6.2a, b, and c, respectively. The average distribution is such that the central bin is positioned at $x=0$ and its neighbours are 


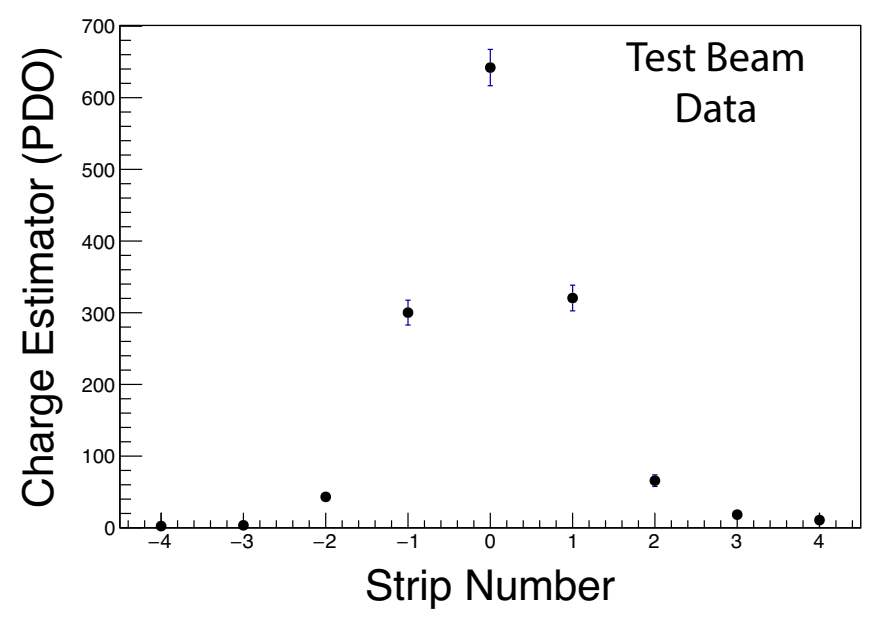

(a)

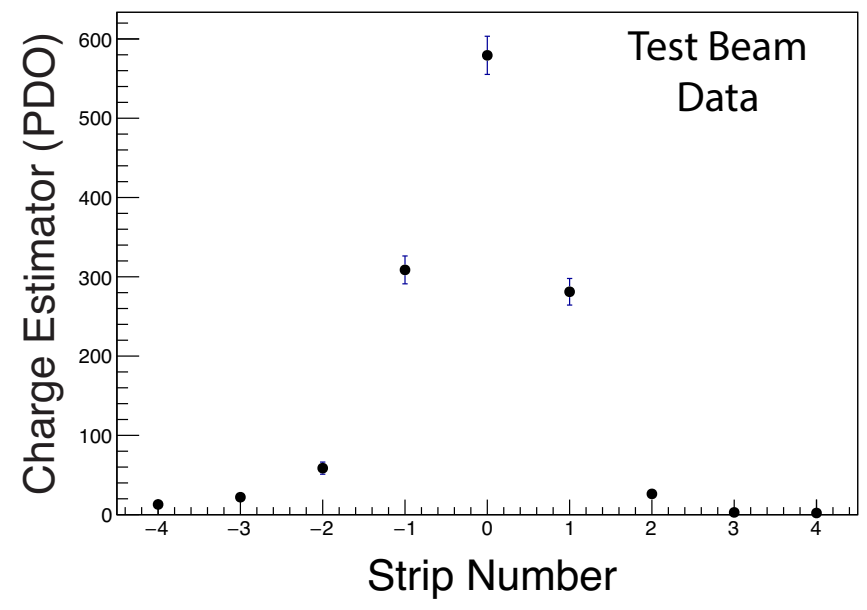

(b)

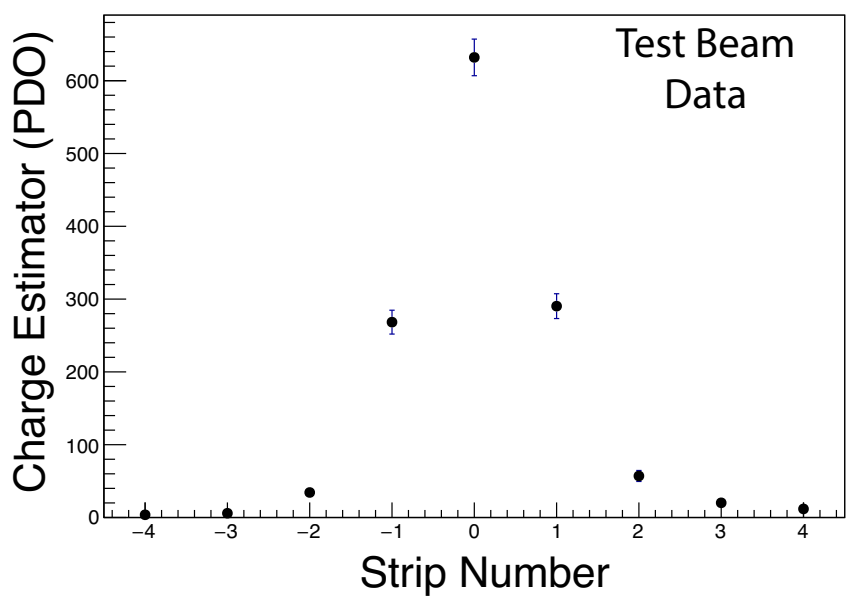

(c)

Figure 6.2: (a) Average charge distribution for layer 1. (b) Average charge distribution for layer 3. (c) Average charge distribution for layer 4. The error bars on the bins correspond to the square root of the bin value. 
plotted around it. The results of the simulated sTGC were compared to these distributions. The simulations are performed with $4.0 \mathrm{GeV}$ muons, however as illustrated in Figure 6.3, the average energy loss in a $2.8 \mathrm{~mm}$ gap filled with 50:50 n-pentane: $\mathrm{CO}_{2}$ remains relatively constant at $1 \mathrm{keV}$ for muons with energy ranging from $1-100 \mathrm{GeV}$. It is expected that the energy loss is similar in 45:55 n-pentane: $\mathrm{CO}_{2}$.

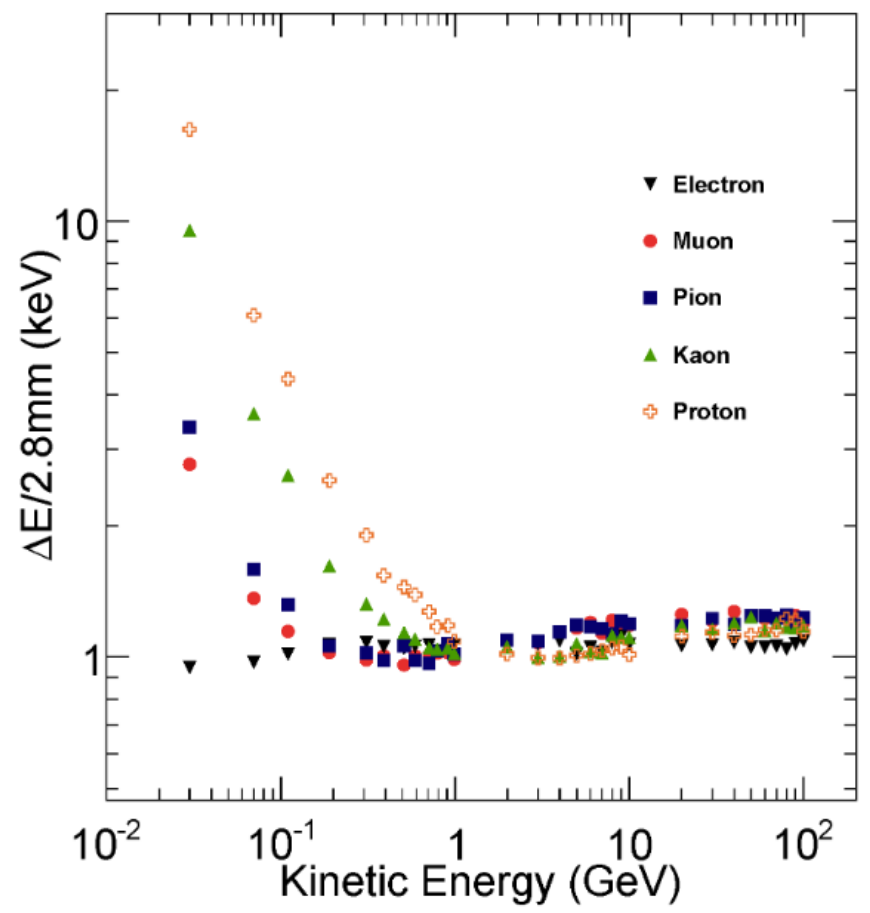

Figure 6.3: The average energy deposited by various particles in a $2.8 \mathrm{~mm}$ gas gap filled with 50:50 n-pentane: $\mathrm{CO}_{2}[35]$.

To obtain a simulated average charge distribution, samples of one thousand simulated muons tracks are carried out with muons passing through the regions between strips 3, 4, and 5. Each sample corresponds to $R C$ values in the range of $21 \mathrm{~ns} / \mathrm{mm}^{2}$ to $120 \mathrm{~ns} / \mathrm{mm}^{2}$ incremented by $1 \mathrm{~ns} / \mathrm{mm}^{2}$ each time. For a given value of $R C$, the central strip is determined in each event and its value, along with the information of the neighbouring strips, are stored in an array with each array element corresponding to the position relative to the central strip. The array is summed over all events and divided over the total number of events to get an average charge distribution for 
the simulated sTGC. The result of the simulations gives the distribution in units of charge (Coulombs) while the test beam data is in terms of PDO. To compare the average distribution from the simulations to the data it is scaled to units of PDO.

To determine the best estimate of $R C$ that fits the simulated results with the test beam data the maximum likelihood method is used. This is given by [36]

$$
\log L(R C)=\sum_{\ell=1}^{N_{\text {bins }}} n_{\ell} \log \nu_{\ell}(R C),
$$

where $n_{\ell}$ corresponds to the value of the test beam distribution in bin $\ell, \nu_{\ell}(R C)$ is the value of the simulated distribution in bin $\ell$ for a given $R C$, and $N_{b i n s}$ is the total number of bins summed over. To determine which value of $R C$ maximizes Equation 6.1 the three most central bins (strip number -1,0, and 1) are fitted. The reasoning for this has been explained in Section 5.1. As mentioned above, the average result from the simulations is fit to each distribution for the three layers and an estimator of $R C$ is obtained for them. These estimators are then combined by summing the likelihood functions for each layer to obtain an overall estimate of $R C$ that best match the simulation to the test beam data. Since the $R C$ time constant controls the dispersion of charge over the strips, the ratios of the total charge on each strip relative to the central strip is compared for the simulation using the best fit estimator and the test beam data.

Furthermore, from the time constant the value of the overall resistance of the resistive layer in the simulations is calculated. Since the capacitance of the coupling between the resistive layer and the strips is considered as the capacitance per unit area the $R C$ constant, in units of $\mathrm{ns} / \mathrm{mm}^{2}$, is equivalent to

$$
\frac{R C}{A}=\frac{R \kappa \epsilon_{0}}{t} .
$$

In Equation $6.2 R C / A$ is the time constant per unit area, $R$ is the resistance of the 
layer, $t$ is the thickness of the combination of the resistive layer and the prepreg in units of $\mu \mathrm{m}, \epsilon_{0}$ is the permittivity of the vacuum equivalent to $8.854 \mathrm{pF} / \mathrm{m}$, and $\kappa$ is the dielectric constant of the gap equivalent to 4.5. The resistance of the layer is calculated by isolating $R$ on the right-hand side of Equation 6.2.

\subsection{Determination of $\mathrm{RC}$}

The determination of the $R C$ time constant follows the analysis procedure outlined above and the results are presented in Figure 6.4. Log-likelihood curves for layers 1, 3 , and 4 are given in Figures 6.4a, b, and c, respectively, where the maximum value of $\log L$ corresponds to the best fit estimator of $R C$. The curve given in Figure 6.4d is the sum of the other three histograms to provide a combined estimator for all three layers. The red line shown in all the plots in Figure 6.4 represents the maximum log-likelihood value subtracted by one-half to provide the range of uncertainty on the respective estimator.

Table 6.1 provides the $R C$ value that corresponds to the maximum of their respective curves along with the uncertainty of each estimate. Furthermore, the calculated resistance corresponding to the best estimate of $R C$ is also given in Table 6.1. The resistance was calculated using a thickness of $200 \mu \mathrm{m}$ for the resistive layer and prepreg. As mentioned previously, the $R C$ value of the QS2 used during the test beam is $40 \mathrm{~ns} / \mathrm{mm}^{2}$ with a resistance of $200 \mathrm{k} \Omega$ at a resistive layer/prepreg thickness of $200 \mu \mathrm{m}$. This value of $R C$ falls within the range of uncertainty of the best fit estimates of layers 1 and 3. For layer 4, the estimated value of $R C$ and its uncertainty are significantly larger than the true time constant value of the tested sTGC. This method will need to be compared to more detailed data to investigate if this discrepancy is a result of the analysis or if the layer could have been malfunctioning or the resistive coating was indeed larger than $200 \mathrm{k} \Omega$. As explained in Section 6.1, the 


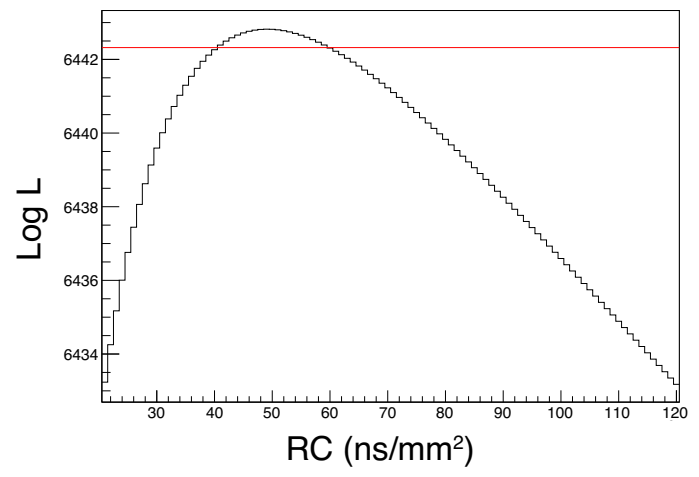

(a)

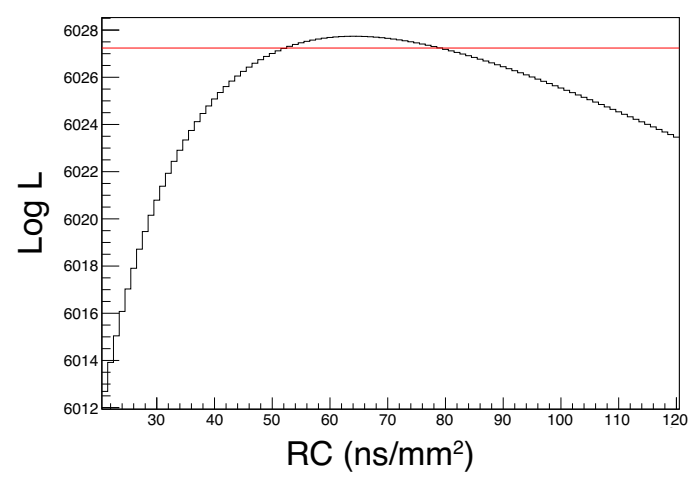

(c)

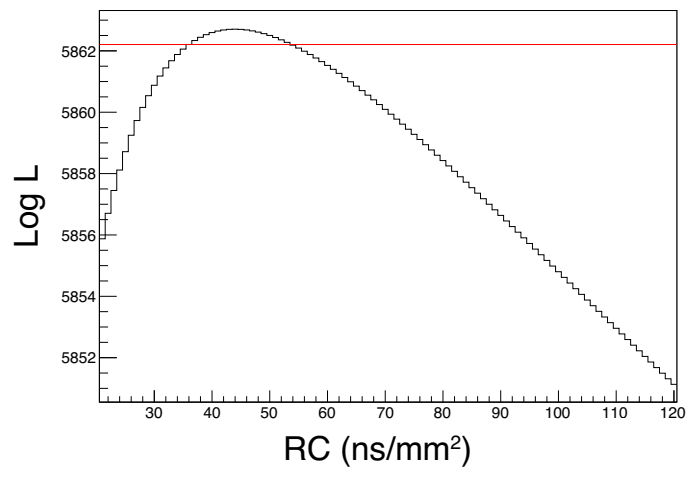

(b)

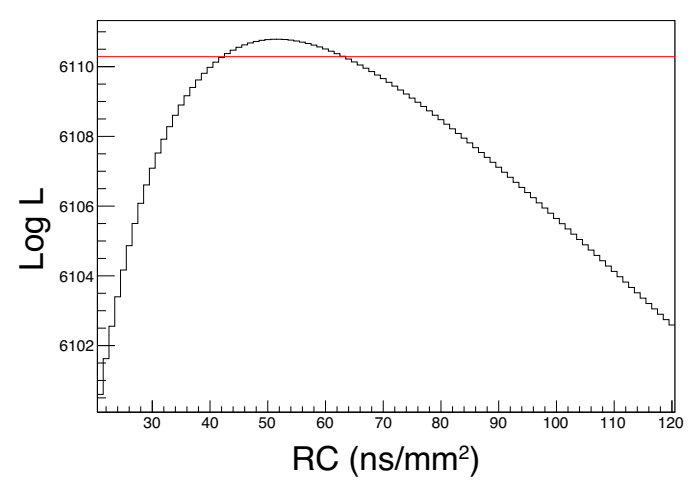

(d)

Figure 6.4: (a) Log-likelihood curve for the best fit of layer 1. (b) Curve used to determine the best fit of layer 3. (c) Best fit curve to determine the $R C$ for layer 4. (d) The result of summing the maximum likelihood curve from each layer obtaining the overall best fit estimator of $R C$ for all layers. The red line in all four plots represented the range of error on the estimator that corresponds to $\log L-1 / 2$.

results from layer 2 have been omitted from the analysis since the recorded data from the layer did not effectively record muon events. The combined estimate of all three layers did not encompass the true value within the range of its uncertainty as layer 4 weighted the estimate towards the higher values of $R C$. 


\begin{tabular}{|c|c|c|}
\hline & $R C$ Estimate & Resistance \\
\hline Layer 1 & $49_{-9}^{+11} \mathrm{~ns} / \mathrm{mm}^{2}$ & $246_{-45}^{+55} \mathrm{k} \Omega$ \\
\hline Layer 3 & $44_{-8}^{+10} \mathrm{~ns} / \mathrm{mm}^{2}$ & $221_{-40}^{+50} \mathrm{k} \Omega$ \\
\hline Layer 4 & $64_{-12}^{+16} \mathrm{~ns} / \mathrm{mm}^{2}$ & $321_{-60}^{+80} \mathrm{k} \Omega$ \\
\hline Combined & $52_{-10}^{+12} \mathrm{~ns} / \mathrm{mm}^{2}$ & $261_{-50}^{+60} \mathrm{k} \Omega$ \\
\hline
\end{tabular}

Table 6.1: The RC time constant values corresponding to the best fit for each layer as well as the combined estimator for all three. The corresponding resistances of the resistive layer were calculated for each best estimate with the uncertainty being obtained by the bounds of uncertainty on the $\mathrm{RC}$ values.

The simulated distributions with the $R C$ value that best fit each layer are overlaid with their respective test beam distributions and presented in Figure 6.5. The charge distribution corresponding to an $R C$ value of $49 \mathrm{~ns} / \mathrm{mm}^{2}$ is shown in Figure 6.5a with the test beam data obtained from layer 1 . Figure $6.5 \mathrm{~b}$ is the overlay of the simulated distribution with a $R C$ value of $44 \mathrm{~ns} / \mathrm{mm}^{2}$ with the experimental charge cluster of layer 3. The results provided in Figure 6.5c correspond to the best fit for layer 4 where the simulated charge distribution uses a $R C$ value of $64 \mathrm{~ns} / \mathrm{mm}^{2}$. The combination of the $R C$ for the three layers corresponds to $52_{-10}^{+12} \mathrm{~ns} / \mathrm{mm}^{2}$. The blue bands in Figure 6.5 correspond to the estimated error on the PDO that is taken to be the square root of the bin value.

The ratio of charge on the neighbouring strips relative to the total charge on the central strip is presented in Table 6.2. It is seen that most of the charge resides within the three central strips of the charge cluster, however, more charge appears to flow to neighbours further out in the simulation compared to the test beam. 


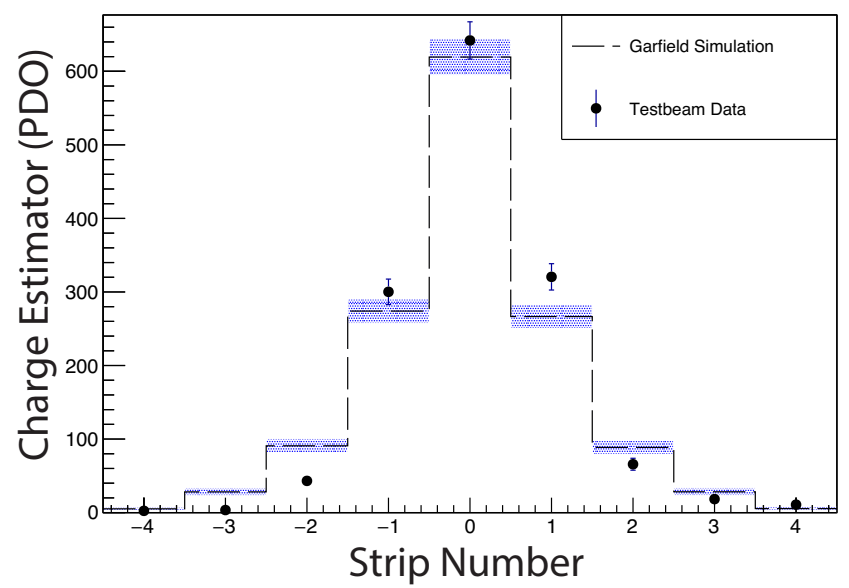

(a)

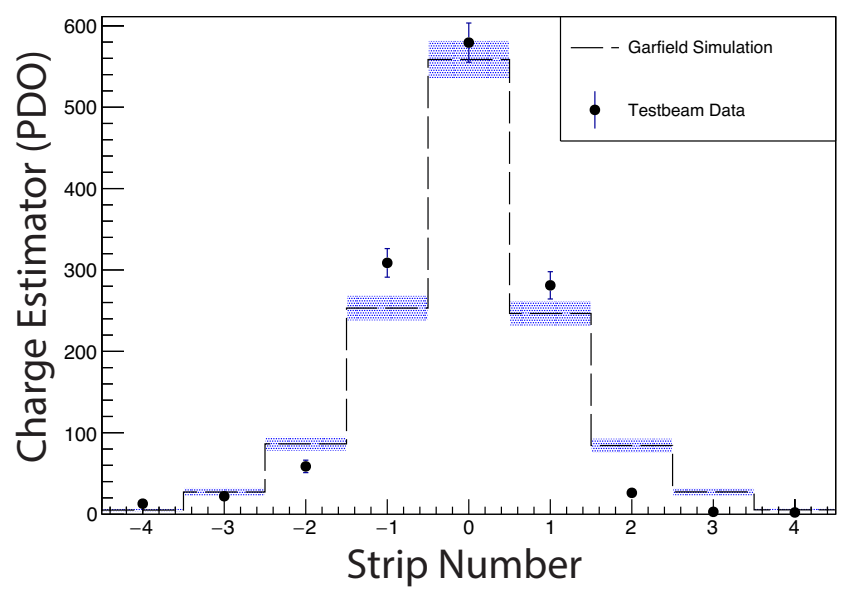

(b)

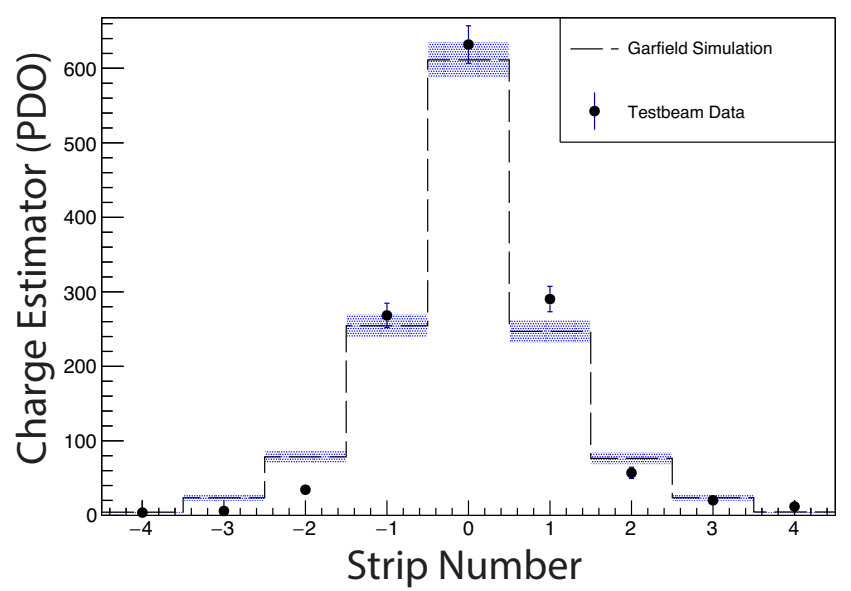

(c)

Figure 6.5: (a) Best fit charge distribution with test beam data from layer 1. (b) The simulated charge distribution that best fit layer 3. (c) Charge distribution corresponding to the best fit value for layer 4 . The blue shaded regions correspond to the uncertainty of the strip PDO for the simulations. 


\begin{tabular}{|l|c|c|c|c|}
\hline & $\mathrm{Q}_{\text {neigh }}^{2 \text { ndleft }} / \mathrm{Q}_{\text {central }}$ & $\mathrm{Q}_{\text {neigh }}^{\text {stleft }} / \mathrm{Q}_{\text {central }}$ & $\mathrm{Q}_{\text {neigh }}^{\text {stright }} / \mathrm{Q}_{\text {central }}$ & $\mathrm{Q}_{\text {neigh }}^{2 \text { ndright }} / \mathrm{Q}_{\text {central }}$ \\
\hline Simulation & $15 \%$ & $44 \%$ & $43 \%$ & $14 \%$ \\
\hline Layer 1 & $7 \% \mathrm{~ns}$ & $47 \%$ & $50 \%$ & $10 \%$ \\
\hline Simulation & $15 \%$ & $45 \%$ & $44 \%$ & $15 \%$ \\
$R C$ 44 ns & $10 \%$ & $53 \%$ & $49 \%$ & $5 \%$ \\
\hline Layer 3 & $13 \%$ & $42 \%$ & $40 \%$ & $13 \%$ \\
\hline Simulation & & & & \\
$R C$ 64 ns & $5 \%$ & $42 \%$ & $46 \%$ & $9 \%$ \\
\hline Layer 4 & & & & \\
\hline
\end{tabular}

Table 6.2: The percentages of the relative charge on the neighbouring strip relative to the total charge on the central strip. 


\section{Chapter 7}

\section{Conclusion \& Outlook}

Simulations of a gaseous detector with the construction parameters of a small segment of the sTGC is shown to have been performed. Each simulation consists of a single $4 \mathrm{GeV}$ muon track passing through the detector. By considering the ionization and transport properties of electron clusters that are produced by the passing muon, the induction of charge on detector elements is simulated using Ramo's theorem to produce raw signals on the electrodes. The response of the sTGC wire to the readout electronics is considered in the formation of the final wire signal. Since the avalanche at the wire induces charge on the surface of the cathode, a model is presented to explain the dispersion of charge over the resistive layer in the sTGC. This model is applied to the simulated raw signals to account for the charge dispersion over the strips. From this, the effects of the readout electronics are applied to the signal on the strips to obtain a final strip signal for the simulation.

The result from the track reconstruction is to be further investigated to determine why the reconstructed $y$-coordinate attains a near constant value for tracks positioned off-centre of a strip. Research into a different function to describe the cluster charge shape over the strip will be performed to attempt to reconcile this discrepancy. Another point of interest to be built up on from this work is the time of arrival of 
the electron cluster to the wire in the situation where the wire nearest to the track might be missing as well as study the timing of the cathode signal to pass the VMM threshold.

By considering the factors involved for the signal formation in the sTGC the results are compared to the test beam data obtained from the ATLAS Muon Group to determine the appropriate $R C$ time constant to be used in the modelling of the resistive layer. The results obtained from comparing layers 1 and 3 are consistent with the construction parameters of the tested sTGC, however, a small discrepancy arose with layer 4 . The overall combined $R C$ value obtained for the fit of the simulated events to the data yields $52_{-10}^{+12} \mathrm{~ns} / \mathrm{mm}^{2}$ which is in agreement with the construction parameter of $40 \mathrm{~ns} / \mathrm{mm}^{2}$. Also, research into the charge sharing ratio of the central strip with its neighbours on an event by event basis will be crucial to obtain agreement during the commissioning phase of the sTGC. To further test the validity and improve on this technique, the simulations will be compared to more detailed real data that will come from the commissioning of the detector until 2021.

The work presented in this thesis illustrates that by considering the factors involved in signal formation, charged particle detectors can be effectively simulated. From this, all our findings are to be implemented in Athena (detailed ATLAS software) for calibration, reconstruction, and simulation. To continue, studies of track pattern recognition are to be investigated to help resolve tracks when two muons simultaneously pass through the detector. The procedure outlined in this study focused on the simulation of a gaseous MWPC, however, this technique can be applied to any charged particle detector where charge is produced on the detector surfaces.

The simulation technique here represents a solid framework to tune the shape of the charge cluster for muon track reconstruction on real data when ATLAS will acquire data with the NSW. 


\section{References}

[1] The ATLAS Collaboration et al., "Observation of a new particle in the search of the Standard Model Higgs boson with the ATLAS detector at the LHC," Physics Letter B, vol. 716, pp. 1-29, Sept. 2012.

[2] M. Tanabashi et al., "Review of particle physics," Phys. Rev. D, vol. 98, p. 030001, Aug. 2018.

[3] AC Team, "The four main LHC experiments." CERN-AC-9906026, Jun. 1999.

[4] J. Pequenao, "Computer generated image of the whole ATLAS detector." CERNGE-0803012, Mar. 2008.

[5] The ATLAS Collaboration et al., "The ATLAS inner detector commissioning and calibration," The European Physical Journal C, vol. 70, pp. 787-821, Dec. 2010 .

[6] S. Morgenstern, "ATLAS LAr Calorimeter Performance in LHC Run-2," Tech. Rep. ATL-LARG-PROC-2018-004, CERN, Geneva, Jun 2018.

[7] H. Correia and A. Maria, "The ATLAS Tile Calorimeter," Tech. Rep. ATLTILECAL-PROC-2015-002, CERN, Geneva, Mar. 2015.

[8] J. Pequenao, "Computer generated image of the ATLAS Muons subsystem." CERN-GE-0803017, Mar. 2008.

[9] T. Argyropoulos et al., "Cathode strip chambers in ATLAS: Installation, commissioning and in situ performance," IEEE Trans. Nucl. Sci., vol. 56, pp. 15681574, 2009.

[10] M. Livan, "Monitored drift tubes in ATLAS," Tech. Rep. ATL-M-PN-129, CERN, Geneva, Sep 1996.

[11] G. Cattani et al., "The resistive plate chambers of the ATLAS experiment: performance studies," Journal of Physics: Conference Series, vol. 280, p. 012001, Feb. 2011.

[12] E. Etzion, Y. Benhammou, J. Ginzburg, M. Ishino, L. Levinson, G. Mikenberg, N. Panikashvili, D. Primor, and V. P. Smakhtin, "The Certification of ATLAS Thin Gap Chambers Produced in Israel and China 019," arXiv e-prints, vol. 1, 
pp. 236-241. 6 p, Oct. 2004. Presented at 2004 IEEE Nuclear Science Symposium 2004, Rome, Oct 2004.

[13] ATLAS Collaboration, "Event display of a $\mathrm{H}$ to $4 \mathrm{mu}$ candidate event." General Photo, Jul. 2012.

[14] T. Kawamoto et al., "New Small Wheel Technical Design Report," Tech. Rep. CERN-LHCC-2013-006. ATLAS-TDR-020, CERN, Jun 2013. ATLAS New Small Wheel Technical Design Report.

[15] F. Kuger, "Micromesh-selection for the atlas new small wheel micromegas detectors," Journal of Instrumentation, vol. 11, pp. C11043-C11043, 112016.

[16] A. Zibell, "Micromegas detectors for the upgrade of the atlas muon spectrometer," Journal of Instrumentation, vol. 9, p. C08013, 082014.

[17] J. Jackson, Classical Electrodynamics, 3rd ed. Wiley India Pvt. Limited, 2007.

[18] W. Blum, W. Riegler, and L. Rolandi, Particle Detection with Drift Chambers 2nd Ed. Springer, 2008.

[19] T. Heubrandtner, B. Schnizer, C. Lippman, and W. Riegler, "Static electric fields in an infinite plane condenser with one or three homogenous layers," Nuclear Instruments and Methods in Physics Research Section A: Accelerators, Spectrometers, Detectors and Associated Equipment, vol. 489, pp. 439 - 443, Aug. 2002.

[20] S. Ramo, "Currents induced by electron motion," Proceedings of the IRE, vol. 27, pp. $584-585$, Sept. 1939.

[21] M. Hamid and S. Bri, "Micromegas detector using 55 fe x-ray source," International Journal of Advanced Research, vol. 1, 012013.

[22] F. Sauli, Gaseous Radiation Detectors: Fundamentals and Applications. Cambridge Monographs on Particle Physics, Nuclear Physics and Cosmology, Cambridge University Press, 2014.

[23] R. Fernow, Introduction to Experimental Particle Physics. Cambridge University Press, 1989.

[24] W. Leo, Techniques for Nuclear and Particle Physics Experiments: A How-to Approach. Springer, 1994.

[25] Y. Assran and A. Sharma, "Transport Properties of operational gas mixtures used at LHC," Tech. Rep. arXiv:1110.6761, CERN, Nov 2011. Comments: 35 pages.

[26] F. Sauli, "Principles of operation of multiwire proportional and drift chambers," in Lectures given in the Academic Training Programme of CERN 1975-1976, (Geneva), p. 92 p, CERN, CERN, 1977. CERN, Geneva, 1975 - 1976. 
[27] A. Bellerive, "Status of the ILC-TPC," in 14th Conference on Astroparticle, Particle, and Space Physics (ICATPP), Italy (p. 445-449), World Scientific Publishing Company, 2014.

[28] The ILD Concept Group, "The international large detector: Letter of intent. 163." ILC Global Design Effort, 2010.

[29] M. Dixit and A. Rankin, "Simulating the charge dispersion phenomena in micro pattern gas detectors with a resistive anode," Nuclear Instruments and Methods in Physics Research Section A: Accelerators, Spectrometers, Detectors and Associated Equipment, vol. 566, no. 2, pp. 281 - 285, 2006.

[30] I. Roth, A. Klier, and E. Duchovni, "Testing stgc with small angle wire edges for the atlas new small wheel muon detector upgrade," in 2015 4th International Conference on Advancements in Nuclear Instrumentation Measurement Methods and their Applications (ANIMMA), pp. 1-6, Apr. 2015.

[31] A. Abusleme et al., "Performance of a full-size small-strip thin gap chamber prototype for the atlas new small wheel muon upgrade," Nuclear Instruments and Methods in Physics Research Section A: Accelerators, Spectrometers, Detectors and Associated Equipment, vol. 817, pp. 85 - 92, 2016.

[32] S. Majewski et al., "A thin multiwire chamber operating in the high multiplication mode," Nuclear Instruments and Methods in Physics Research, vol. 217, no. 1, pp. $265-271,1983$.

[33] R. Veenhof, "Garfield 9." http://garfield.web.cern.ch/garfield/, 2010.

[34] N. Majumdar and S. Mukhopadhyay, "Simulation of three-dimensional electrostatic field configuration in wire chambers: A novel approach," Nuclear Instruments and Methods in Physics Research Section A: Accelerators, Spectrometers, Detectors and Associated Equipment, vol. 566, no. 2, pp. 489 - 494, 2006.

[35] L. Guan, Development of Advanced Gaseous Detectors for Muon Tracking and Triggering in Collider Experiments. PhD thesis, University of Science and Technology of China, Sep. 2014. Presented 16 Oct. 2014.

[36] G. Cowan, Statistical Data Analysis. Oxford science publications, Clarendon Press, 1998. 


\section{Appendix A}

\section{Resistive Layer Response Functions}

Each strip has six neighbours therefore seven variations of the response function $R_{k}(t)$ were used. Each function describes the charge that flows across itself $(k=0)$, the charge that flows to its nearest neighbour $(k=1)$, all the way to the charge that flows to its furthest neighbour $(k=6)$. Below are the histograms of the seven functions.

$$
\text { 1. } R_{0}(t)=\frac{1}{\pi}\left[\tan ^{-1}\left(\frac{w / 2}{2 \sqrt{h t}}\right)-\tan ^{-1}\left(\frac{-w / 2}{2 \sqrt{h t}}\right)\right]
$$

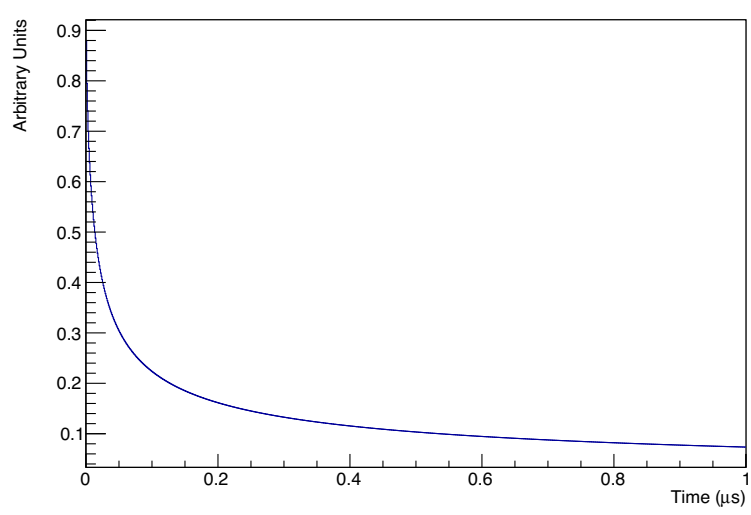


2. $R_{1}(t)=\frac{1}{\pi}\left[\tan ^{-1}\left(\frac{3 w / 2}{2 \sqrt{h t}}\right)-\tan ^{-1}\left(\frac{w / 2}{2 \sqrt{h t}}\right)\right]$

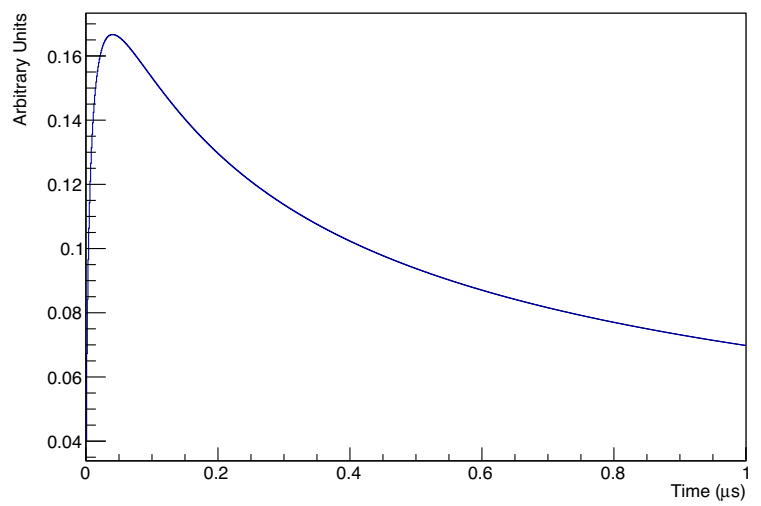

3. $R_{2}(t)=\frac{1}{\pi}\left[\tan ^{-1}\left(\frac{5 w / 2}{2 \sqrt{h t}}\right)-\tan ^{-1}\left(\frac{3 w / 2}{2 \sqrt{h t}}\right)\right]$

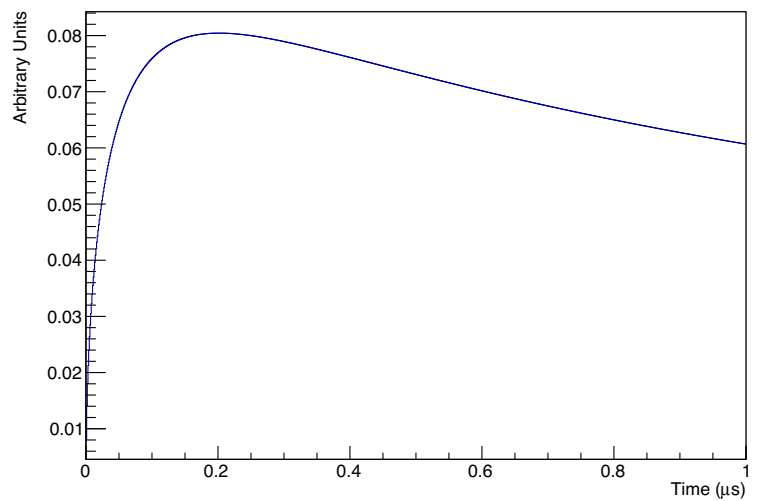

4. $R_{3}(t)=\frac{1}{\pi}\left[\tan ^{-1}\left(\frac{7 w / 2}{2 \sqrt{h t}}\right)-\tan ^{-1}\left(\frac{5 w / 2}{2 \sqrt{h t}}\right)\right]$

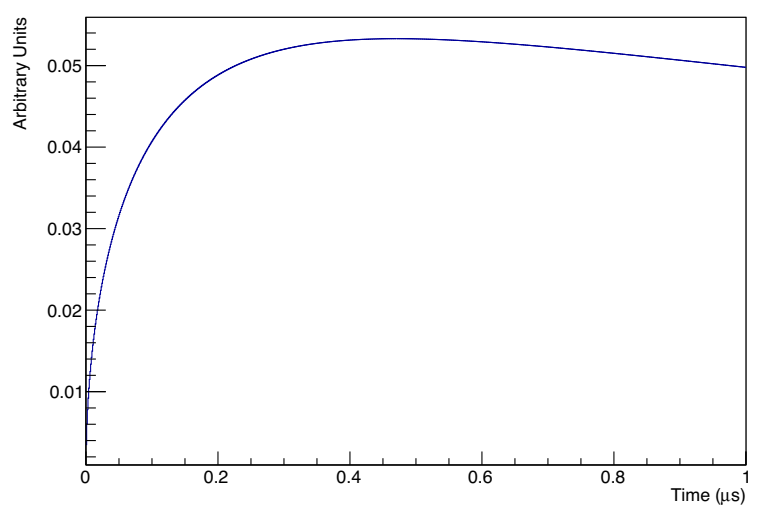


5. $R_{4}(t)=\frac{1}{\pi}\left[\tan ^{-1}\left(\frac{9 w / 2}{2 \sqrt{h t}}\right)-\tan ^{-1}\left(\frac{7 w / 2}{2 \sqrt{h t}}\right)\right]$

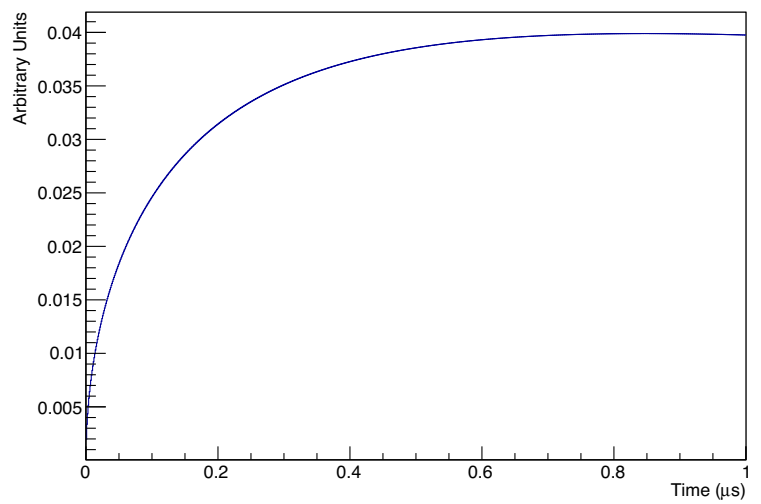

6. $R_{5}(t)=\frac{1}{\pi}\left[\tan ^{-1}\left(\frac{11 w / 2}{2 \sqrt{h t}}\right)-\tan ^{-1}\left(\frac{9 w / 2}{2 \sqrt{h t}}\right)\right]$

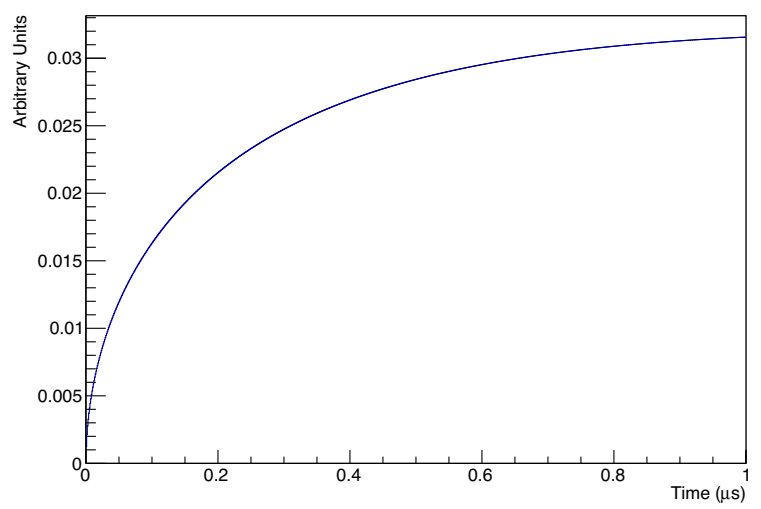

7. $R_{6}(t)=\frac{1}{\pi}\left[\tan ^{-1}\left(\frac{13 w / 2}{2 \sqrt{h t}}\right)-\tan ^{-1}\left(\frac{11 w / 2}{2 \sqrt{h t}}\right)\right]$

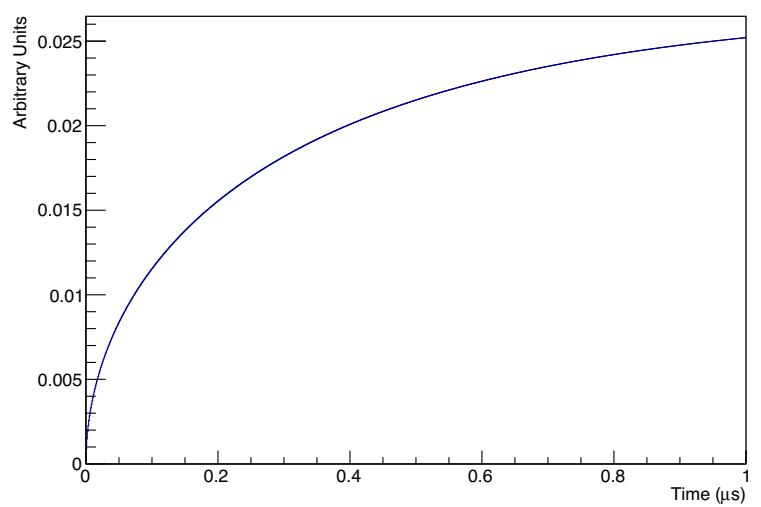

In $R_{k}(t), w$ is defined as the strip width of $3.2 \mathrm{~mm}$. The subscripts $0, \ldots, 6$ represent what neighbour the specific response function corresponds to with 0 being the strip itself. 